\title{
Elementary totally disconnected locally compact groups
}

\author{
Phillip Wesolek
}

\begin{abstract}
We identify the class of elementary groups: the smallest class of totally disconnected locally compact second countable (t.d.l.c.s.c.) groups that contains the profinite groups and the discrete groups, is closed under group extensions of profinite groups and discrete groups, and is closed under countable increasing unions. We show this class enjoys robust permanence properties. In particular, it is closed under group extension, taking closed subgroups, taking Hausdorff quotients, and inverse limits. A characterization of elementary groups in terms of well-founded descriptive-set-theoretic trees is then presented. We conclude with three applications. We first prove structure results for general t.d.l.c.s.c. groups. In particular, we show a compactly generated t.d.l.c.s.c. group decomposes into elementary groups and topologically characteristically simple groups via group extension. We then prove two local-to-global structure theorems: Locally solvable t.d.l.c.s.c. groups are elementary and $[A]$-regular t.d.l.c.s.c. groups are elementary.
\end{abstract}

\section{Introduction}

We study totally disconnected locally compact (t.d.l.c.) groups that are also second countable (s.c.). T.d.l.c.s.c. groups are members of the natural and robust class of Polish groups, i.e. topological groups that are separable and completely metrizable; cf. [18, (5.3)]. Polish spaces and, therefore, Polish groups are considered to be the "correct" topological spaces and topological groups to study. That is to say, they capture almost all naturally occurring examples, admit useful and general theorems such as the Baire category theorem, and exclude pathological examples. The second countability assumption is also quite mild. Every t.d.l.c. group is a directed union of open $K_{\sigma}$ subgroups that are second countable modulo a compact normal subgroup; cf. [16, (8.7)]. Our results may therefore be easily adapted to the t.d.l.c. setting.

In the study of t.d.l.c.s.c. groups, groups "built" from profinite groups and discrete groups arise often. Certainly, there are a number of counterexamples built as such. Less obviously, the work of V.P. Platonov [24 demonstrates that a t.d.l.c.s.c. group such that every finite set generates a relatively compact subgroup may be written as a countable increasing union of profinite groups. More subtly still, a residually discrete t.d.l.c.s.c. group may be written as a countable increasing union of SIN groups by results of P-E. Caprace and N. Monod [8].

T.d.l.c.s.c. groups built from profinite groups and discrete groups also seem to play an important role in the structure theory of t.d.l.c.s.c. groups. Indeed, 15 shows the kernel of the adjoint representation of a $p$-adic Lie group is such a group. More generally, the work 8 indicates groups built from profinite groups and discrete groups are the barrier to finding non-trivial minimal normal subgroups, which is an essential step in reducing a t.d.l.c.s.c. group into "basic" groups.

The frequency of occurrence of t.d.l.c.s.c. groups built from profinite groups and discrete groups and the important role they appear to play in the structure of general t.d.l.c.s.c. groups encourage further study. We here initiate this study, make a number of contributions to the theory, and give a few applications. 


\subsection{Statement of results}

We consider a seemingly narrow class of t.d.l.c.s.c. groups built from profinite groups and discrete groups.

Definition 1.1. The class of elementary groups is the smallest class $\mathscr{E}$ of t.d.l.c.s.c. groups such that

(i) $\mathscr{E}$ contains all second countable profinite groups and countable discrete groups;

(ii) $\mathscr{E}$ is closed under taking group extensions of second countable profinite groups and countable discrete groups. I.e. if $G$ is a t.d.l.c.s.c. group and $H \unlhd G$ is a closed normal subgroup with $H \in \mathscr{E}$ and $G / H$ either profinite or discrete, then $G \in \mathscr{E}$; and

(iii) If $G$ is a t.d.l.c.s.c. group and $G=\bigcup_{i \in \omega} O_{i}$ where $\left(O_{i}\right)_{i \in \omega}$ is an $\subseteq$-increasing sequence of open subgroups of $G$ with $O_{i} \in \mathscr{E}$ for each $i$, then $G \in \mathscr{E}$. We say $\mathscr{E}$ is closed under countable increasing unions.

REMARK 1.2. The class $\mathscr{E}$ could more accurately but less efficiently be called the class of topologically elementary groups. We omit "topologically" as it is redundant in light of the fact we are interested in the non-compact topological theory. We apologize for reducing the interesting and complicated classes of discrete groups and profinite groups to the status of "elementary" groups. Of course, such definitional slights are common in mathematics; e.g. the rich and deep theory of finite groups concerns virtually trivial groups.

Our first results show $\mathscr{E}$ satisfies strong permanence properties indicating this class indeed captures our intuitive idea of groups "built" from profinite groups and discrete groups.

THEOREM 1.3. $\mathscr{E}$ enjoys the following permanence properties:

(1) $\mathscr{E}$ is closed under group extension. I.e. if $G$ is a t.d.l.c.s.c. group and $H \unlhd G$ is a closed normal subgroup such that $H, G / H \in \mathscr{E}$, then $G \in \mathscr{E}$.

(2) If $G \in \mathscr{E}, H$ is a t.d.l.c.s.c. group, and $\psi: H \rightarrow G$ is a continuous, injective homomorphism, then $H \in \mathscr{E}$. In particular, $\mathscr{E}$ is closed under taking closed subgroups.

(3) $\mathscr{E}$ is closed under taking quotients by closed normal subgroups.

(4) If $G$ is a residually elementary t.d.l.c.s.c. group, then $G \in \mathscr{E}$. In particular, $\mathscr{E}$ is closed under inverse limits that result in a t.d.l.c.s.c. group.

(5) $\mathscr{E}$ is closed under quasi-products.

(6) $\mathscr{E}$ is closed under local direct products.

(7) If $G$ is a t.d.l.c.s.c. group and $\left(C_{i}\right)_{i \in \omega}$ is an $\subseteq$-increasing sequence of elementary closed subgroups of $G$ such that $N_{G}\left(C_{i}\right)$ is open for each $i$ and $\overline{\bigcup_{i \in \omega} C_{i}}=G$, then $G \in \mathscr{E}$.

We next characterize elementary groups in terms of well-founded descriptive-set-theoretic trees. This characterization provides not only a tool to identify elementary groups in nature but also a new rank on elementary groups. Using this new rank, we obtain an additional permanence property.

Theorem 1.4. Suppose $H \in \mathscr{E}, G$ is a t.d.l.c.s.c. group, and $\psi: H \rightarrow G$ is a continuous, injective homomorphism. If $\psi(H)$ is normal and dense in $G$, then $G \in \mathscr{E}$.

Our investigations conclude with three applications. The first application is to the general structure theory of t.d.l.c.s.c. groups. We begin by isolating two canonical normal subgroups. 
TheOrem 1.5. Let $G$ be a t.d.l.c.s.c. group. Then

(1) There is a unique maximal closed normal subgroup $\operatorname{Rad}_{\mathscr{E}}(G)$ such that $\operatorname{Rad}_{\mathscr{E}}(G)$ is elementary.

(2) There is a unique minimal closed normal subgroup $\operatorname{Res}_{\mathscr{E}}(G)$ such that $G / \operatorname{Res}_{\mathscr{E}}(G)$ is elementary.

We call $\operatorname{Rad}_{\mathscr{E}}(G)$ and $\operatorname{Res}_{\mathscr{E}}(G)$ the elementary radical and elementary residual, respectively.

Using the elementary radical, a result of Caprace and Monod, and a clever technique of V.I. Trofimov, we arrive at a compelling structure result for compactly generated t.d.l.c.s.c. groups.

TheOREM 1.6. Let $G$ be a compactly generated t.d.l.c.s.c. group. Then there is a finite series of closed characteristic subgroups

$$
\{1\}=H_{0} \leqslant H_{1} \leqslant \ldots \leqslant H_{n} \leqslant G
$$

such that

(1) $G / H_{n} \in \mathscr{E}$, and

(2) for $0 \leqslant k \leqslant n-1,\left(H_{k+1} / H_{k}\right) / \operatorname{Rad}_{\mathscr{E}}\left(H_{k+1} / H_{k}\right)$ is a quasi-product with $0<n_{k+1}<\infty$ many topologically characteristically simple non-elementary quasi-factors.

Theorem [1.6 seems to be convincing evidence that elementary groups are fundamental building blocks of t.d.l.c.s.c. groups. Indeed, Theorem [1.6 demonstrates that the study of compactly generated t.d.l.c.s.c. groups reduces to the study of elementary groups and topologically characteristically simple non-elementary groups.

Since there is interest in compactly generated t.d.l.c. groups, we note a corollary that relaxes the second countability hypothesis.

Corollary 1.7. Let $G$ be a compactly generated t.d.l.c. group. Then there is a finite series of closed normal subgroups

$$
\{1\} \leqslant H_{0} \leqslant H_{1} \leqslant \ldots \leqslant H_{n} \leqslant G
$$

such that

(1) $H_{0}$ is compact and $G / H_{0}$ is second countable,

(2) $G / H_{n} \in \mathscr{E}$, and

(3) for $0 \leqslant k \leqslant n-1,\left(H_{k+1} / H_{k}\right) / \operatorname{Rad}_{\mathscr{E}}\left(H_{k+1} / H_{k}\right)$ is a quasi-product with $0<n_{k+1}<\infty$ many topologically characteristically simple non-elementary quasi-factors.

REMARK 1.8. We provide examples showing $n$ can be arbitrarily large and the topologically characteristically simple quasi-factors cannot be taken to be topologically simple.

In the non-compactly generated case, we obtain a much weaker, nevertheless quite useful structure result; cf. [29.

Definition 1.9. A t.d.l.c.s.c. group is said to be elementary-free if it has no non-trivial elementary closed normal subgroups and no non-trivial elementary Hausdorff quotients. 
TheOREM 1.10. Let $G$ be a t.d.l.c.s.c. group. Then there is a sequence of closed characteristic subgroups

$$
\{1\} \leqslant N \leqslant Q \leqslant G
$$

such that $N$ and $G / Q$ are elementary and $Q / N$ is elementary-free.

The next two applications are so called local-to-global structure results. These are structure results for t.d.l.c. groups with hypotheses on the compact open subgroups. Over the last two decades, there has been a compelling number of such theorems; cf. [2], 6], 9], [10, [30. We first consider groups with an open solvable subgroup.

THEOREM 1.11. If $G$ is a t.d.l.c.s.c. group and $G$ has an open solvable subgroup, then $G \in \mathscr{E}$.

We then consider $[A]$-regular t.d.l.c.s.c. groups. These groups are identified in [9] and are defined by a technical condition on the compact open subgroups. These groups are of interest as they are the barrier to applying the powerful new theory developed in [9].

TheOREM 1.12. If $G$ is a t.d.l.c.s.c. group that is $[A]$-regular, then $G \in \mathscr{E}$.

REMARK 1.13. There are already a number of further applications of the theory of elementary groups. In [28, the author shows a large group extension stable superclass of $\mathscr{E}$ is contained in the class $\mathscr{X}$. The class $\mathscr{X}$ is the class of locally compact groups for which relative amenable closed subgroups are amenable; $\mathscr{X}$ is identified by Caprace and Monod in [7. In a second application [29, the author shows l.c.s.c. $p$-adic Lie groups can be decomposed into elementary groups and compactly generated topologically simple groups via group extension. Contributing to this line of study, H. Glöckner [14] obtains detailed structure theorems for elementary $p$-adic Lie groups.

Acknowledgements. Many of the results herein form part of author's thesis work at the University of Illinois at Chicago. The author wishes to thank his thesis adviser Christian Rosendal and the University of Illinois at Chicago. The author also thanks Pierre-Emmanuel Caprace for his many helpful remarks. The author finally thanks François Le Maître for suggesting improvements to the proof of Theorem 7.10 and the anonymous referee for his or her detailed suggestions.

\subsection{Structure of the paper}

Section 2 lists the background material necessary for our arguments. The reader familiar with the literature on t.d.l.c. groups may safely skip Section 2 with the exception of Subsection 2.4 where the notion of a synthetic subgroup is developed. Section 3 establishes the first permanence properties of the class of elementary groups. We then characterize elementary groups in Section 4 via descriptive-set-theoretic trees. This characterization gives a new rank, the decomposition rank, which allows for more subtle induction arguments. Section 5 uses the decomposition rank to prove an additional permanence property. We present a number of examples in Section 6, We encourage the reader to periodically look ahead to Section [6 in particular, after reading the definition of the decomposition rank, it is enlightening to see the computations of this rank performed in Section 6. We finally consider applications. The 
applications span Sections 7, 8, and 9. The applications do not rely on one another, so the reader may skip any of these sections.

\section{Generalities on totally disconnected locally compact groups}

We begin with a brief overview of necessary background. The notations, definitions, and facts discussed here are used frequently and, typically, without reference.

\subsection{Notations}

All groups are taken to be Hausdorff topological groups and are written multiplicatively. Topological group isomorphism is denoted $\simeq$. We use "t.d.", "l.c.", and "s.c." for "totally disconnected", "locally compact", and "second countable", respectively.

For a topological group $G, S(G)$ and $\mathcal{U}(G)$ denote the collection of closed subgroups of $G$ and the collection of compact open subgroups of $G$, respectively. All subgroups are taken to be closed unless otherwise stated. We write $H \leqslant_{o} G$ and $H \leqslant_{c c} G$ to indicate $H$ is an open subgroup and a cocompact subgroup of $G$, respectively. Recall $H \leqslant G$ is cocompact if the quotient space $G / H$ is compact in the quotient topology.

For a subset $K \subseteq G, C_{G}(K)$ denotes the collection of elements of $G$ that centralize every element of $K$. We denote the collection of elements of $G$ that normalize $K$ by $N_{G}(K)$. The topological closure of $K$ in $G$ is denoted by $\bar{K}$. For $A, B \subseteq G$, we put

$$
\begin{gathered}
A^{B}:=\left\{b a b^{-1} \mid a \in A \text { and } b \in B\right\}, \\
\left.[A, B]:=\left\langle a b a^{-1} b^{-1}\right| a \in A \text { and } b \in B\right\rangle, \text { and } \\
A^{n}:=\left\{a_{1} \ldots a_{n} \mid a_{i} \in A\right\} .
\end{gathered}
$$

For $a, b \in G,[a, b]:=a b a^{-1} b^{-1}$.

If $X$ is a set and $G$ acts on $X$, we write $G \curvearrowright X$. For $g \in G$ and $x \in X, g \cdot x$ denotes the action of $g$ on $x$. For $x \in X$, the stabilizer of $x$ in $G$ is denoted $G_{(x)}$.

If $D$ is a countable discrete group and $X$ a countable set, we put

$$
D^{<X}:=\left\{f \in D^{X} \mid f(x)=1 \text { for all but finitely many } x\right\} .
$$

The group $D^{<X}$ is again a countable discrete group.

We consider 0 to be a limit ordinal. The first countable transfinite ordinal is denoted by $\omega$; as we consider $\mathbb{N}$ to contain zero, $\omega=\mathbb{N}$ as linear orders. The first uncountable ordinal is denoted by $\omega_{1}$. This work will make frequent use of ordinal numbers; see [20] for a nice introduction to ordinal numbers and ordinal arithmetic.

\subsection{Basic theory}

The foundational theorem in the study of t.d.l.c. groups is an old result of D. van Dantzig.

Theorem 2.1 (van Dantzig [16, (7.7)]). A t.d.l.c. group admits a basis at 1 of compact open subgroups.

It follows from van Dantzig's theorem that compact t.d.l.c. groups are profinite; in particular, the compact open subgroups of a t.d.l.c. group are profinite. Since profinite groups are inverse limits of finite groups, they admit a basis at 1 of open normal subgroups. We say $\left(U_{i}\right)_{i \in \omega}$ is a normal basis at 1 for a profinite group $U$, if $U_{0}=U,\left(U_{i}\right)_{i \in \omega}$ is $\subseteq$-decreasing with trivial intersection, and for each $i, U_{i} \unlhd_{o} U$. 
The obvious topological analogues of the familiar isomorphism theorems for groups hold for t.d.l.c.s.c. groups with the exception of the first isomorphism theorem. We thus recall its statement.

Theorem 2.2 ([16, (5.33)]). Let $G$ be a t.d.l.c.s.c. group, $A \leqslant G$ a closed subgroup, and $H \unlhd G$ a closed normal subgroup. If $A H$ is closed, then $A H / H \simeq A /(A \cap H)$ as topological groups.

In the category of t.d.l.c.s.c. groups, care must be taken with infinite unions. Suppose $\left(G_{i}\right)_{i \in \omega}$ is a countable increasing sequence of t.d.l.c.s.c. groups such that $G_{i} \leqslant_{o} G_{i+1}$ for each $i$. The group $G:=\bigcup_{i \in \omega} G_{i}$ becomes a t.d.l.c.s.c. group under the inductive limit topology: $A \subseteq G$ is defined to be open if and only if $A \cap G_{i}$ is open in $G_{i}$ for each $i$. We remark that the condition $G_{i} \leqslant{ }_{o} G_{i+1}$ is required.

Our notion of infinite union allows us to define an infinite direct product that stays in the category of t.d.l.c.s.c. groups.

Definition 2.3. Suppose $A$ is a countable set, $\left(G_{a}\right)_{a \in A}$ is a sequence of t.d.l.c.s.c. groups, and for each $a \in A$, there is a distinguished $U_{a} \in \mathcal{U}\left(G_{a}\right)$. Letting $\left\{a_{i}\right\}_{i \in \omega}$ enumerate $A$, set

- $S_{0}:=\prod_{i \in \omega} U_{a_{i}}$ with the product topology and

- $S_{n+1}:=G_{a_{0}} \times \cdots \times G_{a_{n}} \times \prod_{i \geqslant n+1} U_{a_{i}}$ with the product topology.

The local direct product of $\left(G_{a}\right)_{a \in A}$ over $\left(U_{a}\right)_{a \in A}$ is defined to be

$$
\bigoplus_{a \in A}\left(G_{a}, U_{a}\right):=\bigcup_{n \in \omega} S_{n}
$$

with the inductive limit topology.

The isomorphism type of a local direct product is independent of the enumeration of $A$ used in the definition. Since $S_{n} \leqslant_{o} S_{n+1}$ for each $n \in \omega, \bigoplus_{a \in A}\left(G_{a}, U_{a}\right)$ is again a t.d.l.c.s.c. group with $\prod_{a \in A} U_{a}$ as a compact open subgroup.

We mention an alternative definition of the local direct product: let $\left(G_{a}\right)_{a \in A}$ and $\left(U_{a}\right)_{a \in A}$ be as in Definition 2.3. The local direct product of $\left(G_{a}\right)_{a \in A}$ over $\left(U_{a}\right)_{a \in A}$ is the collection of functions $f: A \rightarrow \bigsqcup_{a \in A} G_{a}$ so that $f(a) \in G_{a}$ for all $a \in A$ and for all but finitely many $a \in A$, $f(a) \in U_{a}$. The topology is given by declaring $\prod_{a \in A} U_{a}$ to be open.

This definition of the local direct product, while obfuscating the topological structure, is more useful when building examples. Indeed, suppose the $G_{a}$ are copies of the same t.d.l.c.s.c. group $G$ and the $U_{a}$ are copies of $U \in \mathcal{U}(G)$. Suppose further $H$ is a t.d.l.c.s.c. group with a continuous action by permutations on $A$. The group $H$ now has a continuous action by topological group automorphisms on $\bigoplus_{a \in A}\left(G_{a}, U_{a}\right)$ by shifting the coordinates: for $h \in H$ and $f \in \bigoplus_{a \in A}\left(G_{a}, U_{a}\right)$, the element $h$ acts on $f$ by $h . f(a):=f\left(h^{-1} . a\right)$. We call this action the shift action of $H$ on $\bigoplus_{a \in A}\left(G_{a}, U_{a}\right)$. The shift action allows us to form $\bigoplus_{a \in A}\left(G_{a}, U_{a}\right) \rtimes H$, which is again a t.d.l.c.s.c. group when given the product topology.

We now recall a number of properties that a t.d.l.c.s.c. group $G$ may display. The group $G$ is said to be compactly generated if there is a compact set $K \subseteq G$ such that $G=\langle K\rangle$. Compactly generated groups have many nice features; we note two here. Any closed cocompact subgroup of a compactly generated group is again compactly generated [21]. The next property we note is a folklore result; a proof is included for completeness. 
Proposition 2.4 (Folklore). Suppose $G$ is a compactly generated t.d.l.c. group, $X$ is a compact generating set, and $U \in \mathcal{U}(G)$. Then there is a finite symmetric set $A \subseteq G$ containing 1 such that $X \subseteq A U$ and $U A U=A U$. Further, for any finite symmetric set $A$ containing 1 with $X \subseteq A U$ and $U A U=A U$, it is the case that $G=\langle A\rangle U$.

Proof. Since $\{x U: x \in X\}$ is an open cover of $X$, we may find $B$ a finite, symmetric set containing 1 such that $X \subseteq B U$. The set $U B$ is again compact, so there is a finite, symmetric $A$ with $B \subseteq A \subseteq U B U$ and $U B \subseteq A U$. We now see that

$$
U A U=U B U \subseteq A U U=A U,
$$

hence, $U A U=A U$. Since $X \subseteq B U \subseteq U B U=U A U=A U$, we have proved the first claim.

Suppose $X \subseteq A U$ and $U A U=A U$ with $A$ a finite symmetric set containing 1 . By induction, $(U A U)^{n}=A^{n} U$ for all $n \geqslant 1$. We conclude that

$$
G=\langle X\rangle=\langle U A U\rangle=\bigcup_{n \geqslant 1}(U A U)^{n}=\bigcup_{n \geqslant 1} A^{n} U=\langle A\rangle U
$$

verifying the second claim.

We say $G$ is a small invariant neighbourhood group, denoted SIN group, if $G$ admits a basis at 1 of compact open normal subgroups. This definition is equivalent to the usual definition of a t.d.l.c. SIN group; cf. [8, Corollary 4.1]. We will make frequent use of the following characterization of compactly generated SIN groups: A compactly generated t.d.l.c. group is SIN if and only if it is residually discrete [8, Corollary 4.1]. Recall a group is residually discrete if every non-trivial element is non-trivial in some discrete quotient. More generally, $G$ is residually $Q$ for $Q$ some property of groups if for all $g \in G \backslash\{1\}$, there is $N \unlhd G$ such that $G / N$ has property $Q$ and the image of $g$ in $G / N$ is non-trivial.

We call $G$ locally elliptic if every finite subset generates a relatively compact subgroup. There is again a useful characterization: A l.c. group is locally elliptic if and only if it is a directed union of compact open subgroups $2 \mathbf{2 4}$.

We say $G$ is a quasi-product with quasi-factors $N_{1}, \ldots, N_{k}$ if $N_{i} \unlhd G$ for each $1 \leqslant i \leqslant k$ and the multiplication map $m: N_{1} \times \cdots \times N_{k} \rightarrow G$ is injective with dense image.

Two subgroups $M \leqslant G$ and $N \leqslant G$ are commensurate, denoted $M \sim_{c} N$, if $|M: M \cap N|$ and $|N: M \cap N|$ are finite. It is easy to check $\sim_{c}$ is an equivalence relation on $S(G)$ and is preserved under the action by conjugation of $G$ on $S(G)$. The commensurability relation allows us to define the commensurator subgroup: For $N \leqslant G$, the commensurator subgroup of $N$ in $G$ is

$$
\operatorname{Comm}_{G}(N):=\left\{g \in G \mid g N g^{-1} \sim_{c} N\right\} .
$$

If $\operatorname{Comm}_{G}(N)=G$, we say $N$ is commensurated.

\subsection{Normal subgroups}

A filtering family $\mathcal{F}$ of subgroups of a group $G$ is a collection of subgroups such that for all $N, M \in \mathcal{F}$, there is $K \leqslant M \cap N$ with $K \in \mathcal{F}$.

Proposition 2.5 (Caprace, Monod [8, Proposition 2.5]). Let $G$ be a compactly generated t.d.l.c. group. Then for any compact open subgroup $V$, the subgroup $Q_{V}:=\bigcap_{g \in G} g V g^{-1}$ is such that any filtering family of non-discrete closed normal subgroups of $G / Q_{V}$ has non-trivial intersection. 
Corollary 2.6. Suppose $G$ is a compactly generated t.d.l.c.s.c. group and $\mathcal{N}$ is a filtering family of closed normal subgroups of $G$. If $\bigcap \mathcal{N}$ is trivial, then some element of $\mathcal{N}$ is compactby-discrete.

Proof. Suppose $\mathcal{N}$ is a filtering family of closed normal subgroups of $G$ such that $\bigcap \mathcal{N}=\{1\}$. The collection $\left\{N^{c} \mid N \in \mathcal{N}\right\}$ is an open cover of $G \backslash\{1\}$, and since $G \backslash\{1\}$ is Lindelöf, there is a countable subcover. We may thus take $\mathcal{N}$ to be a countable filtering family of $G$ such that $\bigcap \mathcal{N}=\{1\}$. It follows from the countability of $\mathcal{N}$ that there is $\left(N_{i}\right)_{i \in \omega}$ an $\subseteq$-decreasing sequence of members of $\mathcal{N}$ such that $\bigcap_{i \in \omega} N_{i}=\{1\}$.

Fix $V \in \mathcal{U}(G)$ and let $Q_{V}$ be as given by Proposition 2.5. Since $Q_{V}$ is compact, $N_{i} Q_{V}$ is a closed normal subgroup of $G$. Furthermore, $\bigcap_{i \in \omega} N_{i} Q_{V}=Q_{V}$. Indeed, take $x \in \bigcap_{i \in \omega} N_{i} Q_{V}$, so $x=n_{i} q_{i} \in N_{i} Q_{V}$ for each $i \in \omega$. Since $Q_{V}$ is compact, there is a subsequence $q_{i_{j}}$ that converges to some $q \in Q_{V}$, whereby $n_{i_{j}}$ also converges to some $n$. For each $k$, we see that $n_{i_{j}} \in N_{k}$ for all $i_{j} \geqslant k$ since $\left(N_{i}\right)_{i \in \omega}$ is decreasing. It follows $n \in N_{k}$ for all $k$ and, therefore, is trivial. Thus, $x \in Q_{V}$.

We now have that $\left(N_{i} Q_{V} / Q_{V}\right)_{i \in \omega}$ is a filtering family in $G / Q_{V}$ with $\bigcap_{i \in \omega} N_{i} Q_{V} / Q_{V}=\{1\}$. Applying Proposition 2.5. some $N_{i} Q_{V} / Q_{V}$ is discrete, and as $N_{i} Q_{V} / Q_{V} \simeq N_{i} /\left(N_{i} \cap Q_{V}\right)$, some $N_{i}$ is compact-by-discrete.

There are a number of canonical normal subgroups that arise in a t.d.l.c.s.c. group $G$. Following M. Burger and S. Mozes [6], the quasi-centre of $G$ is

$$
Q Z(G):=\left\{g \in G \mid C_{G}(g) \text { is open }\right\} .
$$

The subgroup $Q Z(G)$ is a characteristic subgroup but is not necessarily closed; for example, consider the profinite group $F^{\mathbb{N}}$ for $F$ a non-abelian finite simple group [6].

The discrete residual of $G$, denoted $\operatorname{Res}(G)$, is the intersection of all open normal subgroups. The quotient by $\operatorname{Res}(G)$ has a nice structure.

Proposition 2.7. If $G$ is a t.d.l.c.s.c. group, then $G / \operatorname{Res}(G)$ is a countable increasing union of SIN groups.

Proof. Let $\left(g_{i}\right)_{i \in \omega}$ list a countable dense subset of $G$, fix $U \in \mathcal{U}(G)$, and set $O_{i}:=$ $\left\langle U, g_{0}, \ldots, g_{i}\right\rangle$. Certainly, $G=\bigcup_{i \in \omega} O_{i}$, and since $G / \operatorname{Res}(G)$ is residually discrete,

$$
O_{i} \operatorname{Res}(G) / \operatorname{Res}(G) \simeq O_{i} / O_{i} \cap \operatorname{Res}(G)
$$

is residually discrete as well as compactly generated. We conclude that $O_{i} \operatorname{Res}(G) / \operatorname{Res}(G)$ is a SIN group, and the proposition follows.

By work of Platonov 24, a t.d.l.c. group $G$ admits a unique maximal closed normal subgroup that is locally elliptic. This subgroup is called the locally elliptic radical of $G$ and is denoted by $\operatorname{Rad}_{\mathcal{L E}}(G)$.

Following Caprace, C. Reid, and G. Willis [9], the quasi-centralizer of $K$ modulo $L$ for $K, L \leqslant G$ is

$$
Q C_{G}(K / L):=\{g \in G \mid \exists U \in \mathcal{U}(G) \text { such that }[g, U \cap K] \subseteq L\} .
$$

Quasi-centralizers are not necessarily subgroups; however, 
Proposition 2.8 (Caprace, Reid, Willis [9, Lemma 6.5]). Let $G$ be a t.d.l.c. group, let $L$ and $K$ be non-trivial compact subgroups of $G$ such that $L \unlhd K$, and suppose $L$ and $K$ are commensurated. Then $Q C_{G}(K / L)$ is a normal subgroup of $G$ that contains $L$.

The group $Q C_{G}(K / L)$ often fails to be closed, so we define

$$
\overline{Q C}_{G}(K / L):=\overline{Q C_{G}(K / L)} \text {. }
$$

When $K=U$ with $U \in \mathcal{U}(G)$, the compact open subgroup $V:=U \cap \overline{Q C}_{G}(U / L)$ of $\overline{Q C}_{G}(K / L)$ has a useful property: Since $U$ is open, $U \cap Q C_{G}(U / L)$ is dense in $V$, and for each $g \in U \cap$ $Q C_{G}(U / L)$, there is $W \leqslant_{o} U$ such that $[g, w] \in L$ for all $w \in W$. Therefore, $Q Z(V / L)$ is dense in the group $V / L$. This observation will be used in an essential way in Section 9.

\subsection{Synthetic subgroups}

The inchoate idea of a synthetic subgroup appears in work of I.V. Protasov and V.S. Carīn 25. from the late seventies. Specifically, for a t.d.l.c. group $G$ and

$$
P_{1}(G):=\{g \in G \mid \overline{\langle g\rangle} \text { is compact }\},
$$

Protasov and C̆arin define the periodic radical to be the collection of $g \in G$ such that $g P_{1}(G) \subseteq$ $P_{1}(G)$. This collection is easily shown to be a normal subgroup of $G$. We abstract this idea to show a general relationship between sets of closed subgroups and normal subgroups. The key idea we borrow from Protasov and Carīn is to consider the elements that can be added to the members of a collection of subgroups.

For a t.d.l.c. group $G$, a subset $\mathcal{A} \subseteq S(G)$ is conjugation invariant if $\mathcal{A}$ is fixed setwise under the action by conjugation of $G$ on $S(G)$. We say $\mathcal{A}$ is hereditary if for all $C \in \mathcal{A}$, $S(C) \subseteq \mathcal{A}$.

Definition 2.9. Suppose $\mathcal{A} \subseteq S(G)$ is conjugation invariant and hereditary. The $\mathcal{A}$-core, denoted $N_{\mathcal{A}}$, is the collection of $g \in G$ such that for all $C \in \mathcal{A}, \overline{\langle g, C\rangle} \in \mathcal{A}$.

Proposition 2.10. If $\mathcal{A} \subseteq S(G)$ is conjugation invariant and hereditary, then $N_{\mathcal{A}}$ is a normal subgroup of $G$.

Proof. Certainly, $N_{\mathcal{A}}$ is closed under inverses. For multiplication, take $h, g \in N_{\mathcal{A}}$ and $C \in \mathcal{A}$. It is clear that $\overline{\langle h, g, C\rangle} \in \mathcal{A}$, and since $\mathcal{A}$ is hereditary, $\overline{\langle h g, C\rangle} \in \mathcal{A}$. Therefore, $h g \in N_{\mathcal{A}}$, and $N_{\mathcal{A}}$ is a subgroup.

For normality, take $g \in N_{\mathcal{A}}, k \in G$, and $C \in \mathcal{A}$. Since $\mathcal{A}$ is conjugation invariant, we have that $k^{-1} C k \in \mathcal{A}$, so $\overline{\left\langle g, k^{-1} C k\right\rangle} \in \mathcal{A}$. Conjugating with $k$, we see that $\overline{\left\langle k g k^{-1}, C\right\rangle} \in \mathcal{A}$. Therefore, $\mathrm{kgk}^{-1} \in N_{\mathcal{A}}$ verifying that $N_{\mathcal{A}}$ is also normal.

Definition 2.11. A subgroup of a t.d.l.c. group $G$ of the form $N_{\mathcal{A}}$ for some conjugation invariant and hereditary $\mathcal{A} \subseteq S(G)$ is called a synthetic subgroup of $G$.

REMARK 2.12. The technique of synthetic subgroups gives a method to identify normal but not necessarily closed subgroups. Additionally, the closure of a synthetic subgroup often has a regular internal structure that aids in the study of "decompositions".

We give four examples of synthetic subgroups. The latter three appear regularly in this work. 
(1) Let $N \unlhd G$ be a closed normal subgroup of a t.d.l.c. group $G$, so $S(N) \subseteq S(G)$ is conjugation invariant and hereditary. One checks $N$ is the $S(N)$-core.

(2) Let $G$ be a t.d.l.c. group and consider the quasi-centre of $G, Q Z(G)$. Putting

$$
\mathcal{Q Z}:=\left\{C \in S(G) \mid C_{G}(C) \text { is open }\right\},
$$

$Q Z(G)$ is the $\mathcal{Q Z}$-core.

(3) Platonov's locally elliptic radical in the t.d.l.c. setting is an example in a non-trivial manner. Let $G$ be a t.d.l.c. group and $\mathcal{C} \subseteq S(G)$ be the collection of compact subgroups. The set $\mathcal{C}$ is conjugation invariant and hereditary, so we may form $N_{\mathcal{C}}$. As $\{1\} \in \mathcal{C}, \overline{\langle X\rangle}$ is compact for all $X \subseteq N_{\mathcal{C}}$ finite. Via results of $\left[\mathbf{2 4}\right.$ or as an exercise, $\overline{N_{\mathcal{C}}}$ is locally elliptic and, therefore, is contained in $\operatorname{Rad}_{\mathcal{L E}}(G)$. Conversely, take $r \in \operatorname{Rad}_{\mathcal{L E}}(G)$ and $C \in \mathcal{C}$. Since $r^{C} \subseteq \operatorname{Rad}_{\mathcal{L E}}(G)$ is compact, $r^{C}$ generates a compact subgroup. We infer that $\overline{\langle r, C\rangle} \leqslant \overline{\left\langle r^{C}\right\rangle} C$ is a compact subgroup and $r \in N_{\mathcal{C}}$. Therefore, $N_{\mathcal{C}}=\operatorname{Rad}_{\mathcal{L E}}(G)$, and $\operatorname{Rad}_{\mathcal{L E}}(G)$ is the $\mathcal{C}$-core.

(4) Our last example has not been previously isolated. Consider the following family of subgroups in a t.d.l.c.s.c. group $G$ :

$$
\mathcal{S I N}(G):=\left\{C \in S(G) \mid \forall V \in \mathcal{U}(G) \exists W \leqslant_{o} V \text { such that } C \leqslant N_{G}(W)\right\}
$$

We call $\mathcal{S} \mathcal{I} \mathcal{N}(G)$ the $\mathbf{S I N}$-family. The set $\mathcal{S} \mathcal{I} \mathcal{N}(G)$ is conjugation invariant and hereditary, so we may form a synthetic subgroup. We put $\operatorname{SIN}(G):=N_{\mathcal{S I N}(G)}$ where $N_{\mathcal{S I N}(G)}$ is the $\mathcal{S I N}(G)$-core and call $\operatorname{SIN}(G)$ the $\operatorname{SIN}$-core of $G$.

The SIN-core plays an important role in this work, and therefore, we make a few observations. We first note that $Q Z(G) \leqslant \operatorname{SIN}(G)$ and that $\operatorname{SIN}(G)$ is a characteristic subgroup. Our next observation shows the quasi-centre is often a proper subgroup of $\operatorname{SIN}(G)$.

Proposition 2.13. If $G$ is a t.d.l.c.s.c. group, then $\operatorname{SIN}(G)$ is closed.

Proof. Take $C \in \mathcal{S I N}(G)$ and let $U \in \mathcal{U}(G)$ be such that $C \leqslant N_{G}(U)$. For $V \in \mathcal{U}(G)$, we may find $W \unlhd_{o} U$ such that $W \leqslant V$. Since $C \in \mathcal{S I N}(G)$, we may additionally find $L \leqslant_{o} W$ with $C \leqslant N_{G}(L)$. Thus,

$$
\bigcap_{c u \in C U} c u W u^{-1} c^{-1}=\bigcap_{c \in C} c W c^{-1} \geqslant L,
$$

so $\bigcap_{c u \in C U} c u W u^{-1} c^{-1}$ is an open subgroup of $V$ normalized by $C U$. We conclude that $C U \in$ $\mathcal{S I N}(G)$.

Now say $g_{i} \rightarrow g$ with $g_{i} \in \operatorname{SIN}(G)$ for all $i$. Take $C \in \mathcal{S I N}(G)$, find $U \in \mathcal{U}(G)$ so that $C U \in$ $\mathcal{S I N}(G)$, and fix $i$ with $g_{i}^{-1} g \in U$. Since $g_{i} \in \operatorname{SIN}(G)$, we have that $\left\langle g_{i}, C U\right\rangle \in \mathcal{S} \mathcal{I N}(G)$ and by choice of $i,\langle g, C U\rangle \in \mathcal{S I N}(G)$. The hereditary property now implies $\overline{\langle g, C\rangle} \in \mathcal{S I N}(G)$, and the proposition follows.

The SIN-core also has a well-behaved internal structure.

Proposition 2.14. If $G$ is a t.d.l.c.s.c. group, then $\operatorname{SIN}(G)$ is an increasing union of compactly generated relatively open SIN groups.

Proof. Fix $U \in \mathcal{U}(G)$, let $\left(n_{i}\right)_{i \in \omega}$ list a countable dense subset of $N:=\operatorname{SIN}(G)$, and put $H_{i}:=\left\langle N \cap U, n_{0}, \ldots, n_{i}\right\rangle$. Certainly, $N=\bigcup_{i \in \omega} H_{i}$ with $\left(H_{i}\right)_{i \in \omega}$ an $\subseteq$-increasing sequence of compactly generated relatively open subgroups of $N$. It remains to show each $H_{i}$ is a SIN group. To that end, take $O \subseteq H_{i}$ a neighbourhood of 1 in $H_{i}$ and find $W \in \mathcal{U}(G)$ such that $W \cap H_{i} \subseteq O$. We have that $H_{i} \in \mathcal{S} \mathcal{I N}(G)$ by our choice of $n_{0}, \ldots, n_{i}$, so there is $L \leqslant_{o} W$ with 
$H_{i} \leqslant N_{G}(L)$. The subgroup $L \cap H_{i}$ is thus an open normal subgroup of $H_{i}$ contained in $O$ verifying that $H_{i}$ is a SIN group.

\section{Elementary groups}

As it is the central idea of this work, we reiterate the definition of the class of elementary groups.

DEFINITION 3.1. The class of elementary groups is the smallest class $\mathscr{E}$ of t.d.l.c.s.c. groups such that

(i) $\mathscr{E}$ contains all second countable profinite groups and countable discrete groups;

(ii) $\mathscr{E}$ is closed under taking group extensions of second countable profinite groups and countable discrete groups. I.e. if $G$ is a t.d.l.c.s.c. group and $H \unlhd G$ is a closed normal subgroup with $H \in \mathscr{E}$ and $G / H$ either profinite or discrete, then $G \in \mathscr{E}$; and

(iii) If $G$ is a t.d.l.c.s.c. group and $G=\bigcup_{i \in \omega} O_{i}$ where $\left(O_{i}\right)_{i \in \omega}$ is an $\subseteq$-increasing sequence of open subgroups of $G$ with $O_{i} \in \mathscr{E}$ for each $i$, then $G \in \mathscr{E}$. We say $\mathscr{E}$ is closed under countable increasing unions.

\subsection{The construction rank}

For $G \in \mathscr{E}$, define:

- $G \in \mathscr{E}_{0}$ if and only if $G$ is either profinite or discrete.

- Suppose $\mathscr{E}_{\alpha}$ is defined. Put $G \in \mathscr{E}_{\alpha}^{e}$ if and only if there exists $N \unlhd G$ such that $N \in \mathscr{E}_{\alpha}$ and $G / N \in \mathscr{E}_{0}$. Put $G \in \mathscr{E}_{\alpha}$ if and only if $G=\bigcup_{i \in \omega} H_{i}$ where $\left(H_{i}\right)_{i \in \omega}$ is an $\subseteq$-increasing sequence of open subgroups of $G$ with $H_{i} \in \mathscr{E}_{\alpha}$ for each $i \in \omega$. Define $\mathscr{E}_{\alpha+1}:=\mathscr{E}_{\alpha} \cup \mathscr{E}_{\alpha}$.

- For $\lambda$ a limit ordinal, $\mathscr{E}_{\lambda}:=\bigcup_{\beta<\lambda} \mathscr{E}_{\beta}$.

It is easy to verify $\mathscr{E}=\bigcup_{\alpha<\omega_{1}} \mathscr{E}_{\alpha}$. We may thus define

$$
\operatorname{rk}(G):=\min \left\{\alpha \mid G \in \mathscr{E}_{\alpha}\right\} .
$$

We call this rank the construction rank of $G$; the construction rank is inspired by work of D. Osin 23. Induction on the construction rank is one of the primary tools for analysis of elementary groups. The reader is directed to Section 6 to see computations of this rank in examples.

We now collect a number of basic facts about the construction rank. The proof of our first observation is straightforward and, therefore, left to the reader.

OBSERVATION 3.2. Let $G$ be elementary.

(1) If $\operatorname{rk}(G)=\alpha>0$ and $G$ is compactly generated, then $G$ is a group extension of either a profinite group or a discrete group by an elementary group of strictly lower rank.

(2) The construction rank is either zero or a successor ordinal less than $\omega_{1}$.

(3) If $G \simeq H$ as topological groups, then $H \in \mathscr{E}$ and $\operatorname{rk}(G)=\operatorname{rk}(H)$.

Proposition 3.3. If $G \in \mathscr{E}$ and $O \leqslant{ }_{o} G$, then $O \in \mathscr{E}$ and $\operatorname{rk}(O) \leqslant \operatorname{rk}(G)$.

Proof. We induct on $\operatorname{rk}(G)$ for the proposition. As the base case is immediate, suppose the lemma holds for elementary groups with rank at most $\alpha$. Consider a group $G$ with rank $\alpha+1$ and $O \leqslant_{o} G$. Suppose first the rank of $G$ is given by a group extension; say $N \unlhd G$ is so that $\operatorname{rk}(N)=\alpha$ and $\operatorname{rk}(G / N)=0$. Certainly, $\operatorname{rk}(O N / N)=0$, and $O / O \cap N \simeq O N / N$ is either 
profinite or discrete. On the other hand, $O \cap N \leqslant_{o} N$, so the induction hypothesis implies $O \cap N \in \mathscr{E}$ with construction rank at most $\alpha$. We conclude that $O \in \mathscr{E}$ with $\operatorname{rk}(O) \leqslant \alpha+1$.

Suppose the rank of $G$ is given by a union. Say $G=\bigcup_{i \in \omega} H_{i}$ where $\left(H_{i}\right)_{i \in \omega}$ is an $\subseteq$-increasing sequence of open subgroups with $\operatorname{rk}\left(H_{i}\right) \leqslant \alpha$. Certainly, $O=\bigcup_{i \in \omega} O \cap H_{i}$, and applying the induction hypothesis, $O \cap H_{i} \in \mathscr{E}$ with $\operatorname{rk}\left(O \cap H_{i}\right) \leqslant \alpha$ for each $i$. We thus see that $O \in \mathscr{E}$ with $\operatorname{rk}(O) \leqslant \alpha+1$ completing the induction.

Proposition 3.4. If $G \in \mathscr{E}$ and $K \unlhd G$ is compact, then $G / K \in \mathscr{E}$ and $\operatorname{rk}(G / K) \leqslant \operatorname{rk}(G)$.

Proof. We induct on $\operatorname{rk}(G)$ for the proposition. As the base case is obvious, consider $G$ with $\operatorname{rk}(G)=\alpha+1$. Suppose the rank of $G$ is given by a group extension; say $N \unlhd G$ is such that $\operatorname{rk}(N)=\alpha$ and $\operatorname{rk}(G / N)=0$. The group $N K$ is a closed normal subgroup of $G$, and $G / N K$ is either profinite or discrete. On the other hand, applying the induction hypothesis, $N K / K \simeq N /(N \cap K)$ is elementary of rank at most $\alpha$. We now have a short exact sequence of topological groups:

$$
1 \longrightarrow N K / K \longrightarrow G / K \longrightarrow G / N K \longrightarrow 1
$$

The group $G / K$ is thus a group extension of a rank zero group by a rank at most $\alpha$ group, hence $G / K \in \mathscr{E}$ with $\operatorname{rk}(G / K) \leqslant \alpha+1$.

Suppose the rank of $G$ is given by a union. Say $G=\bigcup_{i \in \omega} H_{i}$ with $\left(H_{i}\right)_{i \in \omega}$ an $\subseteq$-increasing sequence of open subgroups with $\operatorname{rk}\left(H_{i}\right) \leqslant \alpha$. We see that $G / K=\bigcup_{i \in \omega} H_{i} K / K$, and the induction hypothesis implies $H_{i} K / K \simeq H_{i} / H_{i} \cap K \in \mathscr{E}$ with

$$
\operatorname{rk}\left(H_{i} / H_{i} \cap K\right) \leqslant \operatorname{rk}\left(H_{i}\right)
$$

for each $i$. Therefore, $G / K \in \mathscr{E}$ with $\operatorname{rk}(G / K) \leqslant \alpha+1$ finishing the induction.

\subsection{Permanence properties}

For the main results of this subsection, we keep track of how the construction rank increases when using a permanence property to produce a new group in $\mathscr{E}$. It is important to remember the arithmetic on the construction ranks is ordinal arithmetic. Arithmetic for transfinite ordinals has a number of peculiarities. For example, it is non-commutative; see [20] for further details.

Proposition 3.5. Let $G$ be a t.d.l.c.s.c. group. If $H \unlhd G$ with $H, G / H \in \mathscr{E}$, then $G \in \mathscr{E}$ and

$$
\operatorname{rk}(G) \leqslant \operatorname{rk}(H)+\operatorname{rk}(G / H)+1 .
$$

Proof. We induct on $\operatorname{rk}(G / H)$ for the proposition. Since the base case holds by definition, consider $G$ with $\operatorname{rk}(G / H)=\alpha+1$. Suppose the rank of $G / H$ is given by an increasing union; that is $G / H=\bigcup_{i \in \omega} O_{i}$ such that $\left(O_{i}\right)_{i \in \omega}$ is an $\subseteq$-increasing sequence of open subgroups with $\operatorname{rk}\left(O_{i}\right) \leqslant \alpha$ for each $i \in \omega$. So $G=\bigcup_{i \in \omega} \pi^{-1}\left(O_{i}\right)$ where $\pi: G \rightarrow G / H$ is the usual projection. Since $\operatorname{rk}\left(\pi^{-1}\left(O_{i}\right) / H\right) \leqslant \alpha$ for each $i \in \omega$, the induction hypothesis implies $\pi^{-1}\left(O_{i}\right) \in \mathscr{E}$ with

$$
\operatorname{rk}\left(\pi^{-1}\left(O_{i}\right)\right) \leqslant \operatorname{rk}(H)+\alpha+1 .
$$

We conclude that $G \in \mathscr{E}$ with $\operatorname{rk}(G) \leqslant \operatorname{rk}(H)+\operatorname{rk}(G / H)+1$.

Suppose the rank of $G / H$ is given by a group extension; say it is given by $L \unlhd G / H$. Certainly, $\pi^{-1}(L) \unlhd G$, and $\operatorname{rk}\left(\pi^{-1}(L) / H\right) \leq \alpha$. Applying the induction hypothesis, $\pi^{-1}(L) \in \mathscr{E}$ with

$$
\operatorname{rk}\left(\pi^{-1}(L)\right) \leqslant \operatorname{rk}(H)+\alpha+1 .
$$


On the other hand, $\operatorname{rk}\left(G / \pi^{-1}(L)\right)=0$, so $G \in \mathscr{E}$ with $\operatorname{rk}(G) \leqslant \operatorname{rk}(H)+\operatorname{rk}(G / H)+1$. This completes the induction, and we conclude the proposition.

REMARK 3.6. Proposition 3.5 shows we may define $\mathscr{E}$ by allowing all group extensions. This makes for a somewhat easier definition to parse; however, the given definition is easier to use in practice.

For our next permanence properties, a subsidiary lemma is required.

LEMma 3.7. Let $G$ be a t.d.l.c.s.c. group.

(1) If $G$ is residually discrete, then $G \in \mathscr{E}$ with $\operatorname{rk}(G) \leqslant 2$.

(2) If $G=\overline{\bigcup_{i \in \omega} L_{i}}$ with $\left(L_{i}\right)_{i \in \omega}$ an $\subseteq$-increasing sequence of closed elementary subgroups of $G$ such that there is $U \in \mathcal{U}(G)$ with $U \leqslant N_{G}\left(L_{i}\right)$ for all $i$, then $G \in \mathscr{E}$.

Proof. For (1), $\operatorname{Res}(G)=\{1\}$, so via Proposition 2.7, $G$ is a countable increasing union of open SIN groups. A SIN group is compact-by-discrete and hence is elementary with rank at most 1 . As $G$ is a countable increasing union of such groups, $G \in \mathscr{E}$ with $\operatorname{rk}(G) \leqslant 2$.

For (2), $U L_{i} \leqslant{ }_{o} G$ is elementary for each $i$, and since $G=\bigcup_{i \in \omega} U L_{i}$, we conclude that $G \in \mathscr{E}$.

THEOREM 3.8. If $G \in \mathscr{E}, H$ is a t.d.l.c.s.c. group, and $\psi: H \rightarrow G$ is a continuous, injective homomorphism, then $H \in \mathscr{E}$ with $\operatorname{rk}(H) \leqslant 3(\operatorname{rk}(G)+1)$. In particular, if $H \leqslant G$ is a closed subgroup, then $H \in \mathscr{E}$ with $\operatorname{rk}(H) \leqslant 3(\operatorname{rk}(G)+1)$.

Proof. We induct on $\operatorname{rk}(G)$. For the base case, $G$ is either profinite or discrete. If $G$ is discrete, then $H$ is discrete and we are done. Suppose $G$ is profinite with $\left(U_{i}\right)_{i \in \omega}$ a normal basis at 1 for $G$. The sequence $\left(\psi^{-1}\left(U_{i}\right)\right)_{i \in \omega}$ thus consists of open normal subgroups of $H$ with trivial intersection. We conclude that $H$ is residually discrete and via Lemma 3.7 that $H \in \mathscr{E}$ with $\operatorname{rk}(H) \leqslant 2$.

Suppose the proposition holds up to construction $\operatorname{rank} \alpha$ and $\operatorname{rk}(G)=\alpha+1$. Suppose first the rank of $G$ is given by an increasing union; say $G=\bigcup_{i \in \omega} O_{i}$ with $\operatorname{rk}\left(O_{i}\right) \leqslant \alpha$ for each $i$. Certainly, $H=\bigcup_{i \in \omega} \psi^{-1}\left(O_{i}\right)$, and the induction hypothesis implies $\psi^{-1}\left(O_{i}\right) \in \mathscr{E}$ with

$$
\operatorname{rk}\left(\psi^{-1}\left(O_{i}\right)\right) \leqslant 3(\alpha+1)
$$

for each $i \in \omega$. We conclude that

$$
\operatorname{rk}(H) \leqslant 3(\alpha+1)+1<3(\alpha+2)
$$

verifying the induction claim in this case.

Suppose $\operatorname{rk}(G)$ is given by a group extension; say $N \unlhd G$ is such that $\operatorname{rk}(N)=\alpha$ and $\operatorname{rk}(G / N)=0$. Since $\psi: \psi^{-1}(N) \rightarrow N$, the induction hypothesis implies $\psi^{-1}(N) \in \mathscr{E}$ with $\operatorname{rk}\left(\psi^{-1}(N)\right) \leqslant 3(\alpha+1)$. On the other hand, $\psi$ induces a continuous, injective map $\tilde{\psi}$ : $H / \psi^{-1}(N) \rightarrow G / N$. If $G / N$ is discrete, then $H / \psi^{-1}(N)$ is discrete and

$$
\operatorname{rk}(H) \leqslant 3(\alpha+1)+1<3(\alpha+2) .
$$

If $G / N$ is profinite, then $H / \psi^{-1}(N)$ is residually discrete as in the base case. The group $H / \psi^{-1}(N)$ is thus an increasing union of open SIN groups, hence $H=\bigcup_{i \in \omega} O_{i}$ with $O_{i} / \psi^{-1}(N)$ a SIN group for each $i$. Applying Proposition 3.5 $O_{i} \in \mathscr{E}$ with

$$
\operatorname{rk}\left(O_{i}\right) \leqslant 3(\alpha+1)+1+1 \text {. }
$$


Since $H=\bigcup_{i \in \omega} O_{i}$, it follows that $H \in \mathscr{E}$ with $\operatorname{rk}(H) \leqslant 3(\alpha+1)+3$. This completes the induction, and we conclude the theorem.

We note an easy but useful corollary.

Corollary 3.9. Suppose $G \in \mathscr{E}$. If $H \leqslant G$ and $\operatorname{rk}(G)<\beta$ for $\beta$ a limit ordinal, then $\operatorname{rk}(H)<\beta$.

Proof. In view of Theorem 3.8 , it suffices to show for any non-zero limit ordinal $\beta$ and ordinal $\gamma<\beta, 3 \gamma+3<\beta$. Letting $\left(\beta_{\alpha}\right)_{\alpha}$ list the non-zero limit ordinals, we induct on $\alpha$. For the base case, $\beta_{0}=\omega$, the result is immediate.

In the case $\beta=\beta_{\lambda}$ with $\lambda$ a limit ordinal, there is $\alpha<\lambda$ so that $\gamma<\beta_{\alpha}$. By the induction hypothesis, we conclude that $3 \gamma+3<\beta_{\alpha}<\beta_{\lambda}$. In the case $\beta=\beta_{\alpha+1}$, there is $m<\omega$ so that $\gamma<\beta_{\alpha}+m$. Hence,

$$
3 \gamma+3<3 \beta_{\alpha}+3 m+3 \leqslant \sup _{\xi<\beta_{\alpha}} 3 \xi+3 m+3=\beta_{\alpha}+3 m+3<\beta_{\alpha+1}
$$

where $\sup _{\xi<\beta_{\alpha}} 3 \xi=\beta_{\alpha}$ follows by the induction hypothesis. This finishes the induction, and we conclude the corollary.

REMARK 3.10. It is easy to build examples of elementary groups $G$ and $H$ such that $H \hookrightarrow G$ and $\operatorname{rk}(H)>\operatorname{rk}(G)$. For example, let $F_{i}$ for $i \in \mathbb{Z}$ list copies of some non-trivial finite group. For $i \leqslant 0$, let $U_{i}=F_{i}$ and for $i>0$, put $U_{i}=1$. We now see $\bigoplus_{i \in \mathbb{Z}}\left(F_{i}, U_{i}\right) \hookrightarrow \prod_{i \in \mathbb{Z}} F_{i}$ with $\operatorname{rk}\left(\bigoplus_{i \in \mathbb{Z}}\left(F_{i}, U_{i},\right)\right)=1$ while $\operatorname{rk}\left(\prod_{i \in \mathbb{Z}} F_{i}\right)=0$. One can build more complicated examples with $\operatorname{rk}(H)-\operatorname{rk}(G)>1$.

We also remark that the bound in Theorem 3.8 is not sharp. For example, the rank zero case of the induction shows if $G$ is profinite, then $\operatorname{rk}(H) \leqslant 2$.

The class of elementary groups is closed under taking quotients. Towards demonstrating this permanence property, we require an additional lemma.

Lemma 3.11. Let $G$ be a t.d.l.c.s.c. group. If $P \unlhd G$ is elementary, $L \unlhd G$, and $[P, L]=\{1\}$, then $\overline{P L} / L \in \mathscr{E}$.

Proof. We induct on $\operatorname{rk}(P)$ for the lemma. For the base case, $\operatorname{rk}(P)=0, P$ is either compact or discrete. When $P$ is compact, $P L$ is closed, so $P L / L \simeq P / P \cap L$ and is elementary. When $P$ is discrete, every element of $P$ has an open centralizer since $P$ discrete and normal. Every element of $P$ in $\overline{P L}$ thus has an open centralizer. It follows

$$
\operatorname{SIN}(\overline{P L} / L)=\overline{P L} / L,
$$

and in view of Proposition 2.14, $\overline{P L} / L$ is elementary.

Suppose $\operatorname{rk}(P)=\alpha+1$. We first reduce to a compactly generated group. Fix $U \in \mathcal{U}(\overline{P L})$, fix $V \in \mathcal{U}(P)$, and let $\left(p_{i}\right)_{i \in \omega}$ list a countable dense subset of $P$. For each $i \in \omega$, set

$$
P_{i}:=\left\langle V^{U}, p_{0}^{U}, \ldots, p_{i}^{U}\right\rangle .
$$

Each $P_{i}$ is normalized by $U$, so $P_{i} U L$ is an open subgroup of $\overline{P L}$. The sequence $\left(P_{i} U L\right)_{i \in \omega}$ is therefore an $(\subseteq)$-increasing exhaustion of $\overline{P L}$ by open subgroups, hence the induction may 
be completed by showing each $P_{i} U L \in \mathscr{E}$. We may indeed reduce further: $\overline{P_{i} L} / L \unlhd_{c c} P_{i} U L / L$, hence the induction will be completed by showing $\overline{P_{i} L} / L \in \mathscr{E}$ for each $i$.

To this end, fix $i \in \omega$, put $Q:=P_{i}$, and set $R:=\overline{Q L}$. We now show $R / L$ is elementary which will verify the induction claim. Since $Q \leqslant{ }_{o} P, \operatorname{rk}(Q) \leqslant \alpha+1$, and since $Q$ is compactly generated, there is $M \unlhd Q$ such that $\operatorname{rk}(M) \leqslant \alpha$ and $\operatorname{rk}(Q / M)=0$. Furthermore, $Q \unlhd R$ and $M \unlhd R$, because $L$ centralizes $P$. Passing to $R / M$, we have that $Q / M \unlhd R / M$ and $\overline{M L} / M \unlhd$ $R / M$. The group $Q / M$ is either profinite or discrete, and $[Q / M, \overline{M L} / M]=\{1\}$. By the base case and construction of $R$,

$$
\overline{(\overline{M L} / M)(Q / M)} /(\overline{M L} / M)=(R / M) /(\overline{M L} / M) \simeq R / \overline{M L}
$$

is elementary.

On the other hand, $\operatorname{rk}(M) \leqslant \alpha,[M, L]=\{1\}$, and $M, L \unlhd R$. The induction hypothesis therefore implies $\overline{M L} / L$ is also elementary. Since

$$
(R / L) /(\overline{M L} / L) \simeq R / \overline{M L} \in \mathscr{E},
$$

we apply Proposition 3.5 to conclude that $R / L$ is elementary. The induction is thus completed.

Theorem 3.12. If $G \in \mathscr{E}$ and $L \unlhd G$, then $G / L \in \mathscr{E}$.

Proof. We induct on $\operatorname{rk}(G)$ for the theorem. As the base case is immediate, we suppose $\operatorname{rk}(G)=\alpha+1$. Consider first the case in which $\operatorname{rk}(G)$ is given by a union. That is $G=\bigcup_{i \in \omega} H_{i}$ with $\left(H_{i}\right)_{i \in \omega}$ an $\subseteq$-increasing sequence of open subgroups each with rank at most $\alpha$. Now $G / L=\bigcup_{i \in \omega} H_{i} L / L$, and since $H_{i} L / L \simeq H_{i} / H_{i} \cap L$ for each $i$, the induction hypothesis implies $H_{i} L / L \in \mathscr{E}$ for each $i$. We conclude that $G / L \in \mathscr{E}$.

Suppose $\operatorname{rk}(G)$ is given by a group extension; say $H \unlhd G$ is such that $\operatorname{rk}(H)=\alpha$ and $\operatorname{rk}(G / H)=0$. Pass to $\tilde{G}:=G / L \cap H$ and let $\tilde{H}$ and $\tilde{L}$ be the images of $H$ and $L$ in $\tilde{G}$, respectively. Since $\operatorname{rk}(H)=\alpha$, the group $\tilde{H}$ is elementary by the induction hypothesis, and the construction of $\tilde{G}$ ensures that $[\tilde{H}, \tilde{L}]=\{1\}$. Lemma 3.11 thus implies $\tilde{H} \tilde{L} / \tilde{L}$ is elementary. Seeing as $\tilde{H} \tilde{L} / \tilde{L} \simeq \overline{H L} / L$, we conclude that $\overline{H L} / L$ is indeed elementary.

On the other hand, $\overline{H L} / H \unlhd G / H$ and $G / H$ is either profinite or discrete. Hence,

$$
(G / H) /(\overline{H L} / H) \simeq G / \overline{H L} \simeq(G / L) /(\overline{H L} / L)
$$

is either profinite or discrete. The group $G / L$ is thus a group extension of either a profinite group or a discrete group by an elementary group and, thus, is elementary. This completes the induction, and we conclude the theorem.

REMARK 3.13. A bound on the construction rank of $G / L$ for $L \unlhd G \in \mathscr{E}$ is given in Corollary 4.20

The reader familiar with the theory of elementary amenable discrete groups recalls that the class of elementary amenable groups can be defined analogously to the class of elementary groups; see [11. Our closure properties hitherto mirror those of the class of elementary amenable groups defined as such. The next theorem, however, is surprisingly disanalogous. Indeed, the analogous statement is patently false in the class of elementary amenable groups.

THEOREM 3.14. If $G$ is a t.d.l.c.s.c. group that is residually elementary, then $G \in \mathscr{E}$ with $\operatorname{rk}(G) \leqslant \sup \{\operatorname{rk}(G / N) \mid N \unlhd G$ with $G / N \in \mathscr{E}\}+3$. 
In particular, $\mathscr{E}$ is closed under inverse limits that result in t.d.l.c.s.c. groups.

Proof. Let $\mathcal{F}$ be the collection of closed normal subgroups of $G$ with elementary quotient. We first claim $\mathcal{F}$ is a filtering family; it suffices to show $\mathcal{F}$ is closed under intersection. Take $N, M \in \mathcal{F}$ and consider $M / M \cap N$. The restriction of the usual projection gives a continuous, injective homomorphism $M / M \cap N \rightarrow G / N$. Since $G / N$ is elementary, Theorem 3.8 implies $M / M \cap N$ is elementary, so $M / M \cap N$ is a closed normal elementary subgroup of $G / N \cap M$ with elementary quotient. As $\mathscr{E}$ is closed under group extension, we conclude that $G / M \cap N \in$ $\mathscr{E}$ and, therefore, that $\mathcal{F}$ is closed under intersection.

Fix $O \leqslant_{o} G$ a compactly generated open subgroup of $G$ and put $\mathcal{F}_{O}:=\{N \cap O \mid N \in \mathcal{F}\}$. For each $N \in \mathcal{F}$, we have that $O N / N \simeq O / N \cap O$ and, therefore, that $O / N \cap O$ is elementary. The family $\mathcal{F}_{O}$ is thus a filtering family of closed normal subgroups of $O$ with elementary quotient. Since $G$ is residually elementary, $\cap \mathcal{F}_{O}=\{1\}$, so Corollary 2.6 implies there is $N \in \mathcal{F}_{O}$ that is profinite-by-discrete and, hence, elementary. The group $O / N$ is also elementary, whereby Proposition 3.5 implies $O$ is elementary with

$$
\operatorname{rk}(O) \leqslant 1+\operatorname{rk}(G / N)+1 \leqslant \operatorname{rk}(G / N)+2 .
$$

Since $G$ has a countable $\subseteq$-increasing exhaustion by such $O, G \in \mathscr{E}$ with

$$
\begin{aligned}
\operatorname{rk}(G) & \leqslant \sup \{\operatorname{rk}(G / N)+2 \mid N \unlhd G \text { with } G / N \in \mathscr{E}\}+1 \\
& \leqslant \sup \{\operatorname{rk}(G / N) \mid N \unlhd G \text { with } G / N \in \mathscr{E}\}+3 .
\end{aligned}
$$

For the inverse limit claim, suppose $\left(C_{\alpha}, \phi_{\alpha}\right)_{\alpha \in I}$ is an inverse system of elementary groups with a t.d.l.c.s.c. group $H$ as the inverse limit. The projection onto each coordinate $\alpha \in I$, $\pi_{\alpha}: H \rightarrow C_{\alpha}$, is a continuous homomorphism into an elementary group $C_{\alpha}$. Theorem 3.8 thus implies $H / \operatorname{ker}\left(\pi_{\alpha}\right)$ is elementary for all $\alpha \in I$. Since

$$
\bigcap_{\alpha \in I} \operatorname{ker}\left(\pi_{\alpha}\right)=\{1\}
$$

$H$ is residually elementary and, therefore, elementary.

We conclude with three easy but useful permanence properties of $\mathscr{E}$; we do not compute bounds for the construction rank as such bounds are not used herein and are easily computed.

Proposition 3.15. If $G$ is a quasi-product with quasi-factors $N_{1}, \ldots, N_{k}$ such that $N_{i} \in \mathscr{E}$ for each $i$, then $G \in \mathscr{E}$.

Proof. We induct on $k$ for the proposition. If $k=1$, then the result obviously holds. Suppose $G$ is a quasi-product with elementary quasi-factors $N_{1}, \ldots, N_{k+1}$. Fix $U \in \mathcal{U}(G)$ and put $M:=$ $\overline{\left\langle N_{1}, \ldots, N_{k}\right\rangle}$. We now have that

$$
G / M \simeq U N_{k+1} M / M \simeq U N_{k+1} /\left(U N_{k+1} \cap M\right) .
$$

The rightmost group is a quotient of an elementary group, hence Theorem 3.12 implies $G / M \simeq$ $U N_{k+1} /\left(U N_{k+1} \cap M\right) \in \mathscr{E}$.

The group $M$, on the other hand, is a quasi-product with elementary quasi-factors $N_{1}, \ldots, N_{k}$. The induction hypothesis thereby implies $M \in \mathscr{E}$, and as $\mathscr{E}$ is closed under group extension, $G \in \mathscr{E}$ completing the induction.

Proposition 3.16. If $\left(G_{n}\right)_{n \in \omega}$ is a sequence of elementary groups with a distinguished $U_{n} \in \mathcal{U}\left(G_{n}\right)$ for each $n$, then $\bigoplus_{n \in \omega}\left(G_{n}, U_{n}\right) \in \mathscr{E}$. 
Proof. By construction, $\bigoplus_{n \in \omega}\left(G_{n}, U_{n}\right)$ is an increasing union of groups of the form

$$
G_{0} \times \cdots \times G_{n} \times \prod_{i \geqslant n+1} U_{i} .
$$

These groups are built via repeated group extension from profinite groups and $G_{0}, \ldots, G_{n}$, so Proposition 3.5 implies $G_{0} \times \cdots \times G_{n} \times \prod_{i \geqslant n+1} U_{i} \in \mathscr{E}$. It now follows that $\bigoplus_{n \in \omega}\left(G_{n}, U_{n}\right) \in$ $\mathscr{E}$.

Proposition 3.17. If $G$ is a t.d.l.c.s.c. group and $\left(C_{i}\right)_{i \in \omega}$ is an $\subseteq$-increasing sequence of elementary subgroups of $G$ such that $N_{G}\left(C_{i}\right)$ is open for each $i$ and $\overline{\bigcup_{i \in \omega} C_{i}}=G$, then $G \in \mathscr{E}$.

Proof. Fix $O \leqslant G$ open and generated by a compact $X \subseteq O$ and let $U \in \mathcal{U}(O)$. Via Proposition 2.4. there is $A \subset O$ finite, symmetric, and containing 1 so that $X \subseteq A U$ and $U A U=A U$. Since $\bigcup_{i \in \omega} C_{i}$ is dense, we may find $B \subseteq C_{i}$ for a large enough $i$ so that $B U=A U$ and $B$ is finite and symmetric and contains 1 . We see that $U B U=B U$, and hence, $O=\langle B\rangle U$.

Find $V \leqslant_{o} U$ with $V \leqslant N_{G}\left(C_{i}\right)$. We see that $\overline{\left\langle B^{V}\right\rangle} \leqslant C_{i} \in \mathscr{E}$, and since $\overline{\left\langle B^{V}\right\rangle} \unlhd_{c c}\left\langle B^{V}\right\rangle V$, we infer that $\left\langle B^{V}\right\rangle V \in \mathscr{E}$. On the other hand, the group $\left\langle B^{V}\right\rangle V$ is finite index in $O$, so we may take the normal core $L$ of $\left\langle B^{V}\right\rangle V$ in $O$. Theorem 3.8 implies $L \in \mathscr{E}$, whence $O$ is elementary-by-finite and, therefore, elementary. Since $G$ is an increasing union of compactly generated open elementary subgroups, $G \in \mathscr{E}$ proving the proposition.

Collecting our results, we have the following theorem.

THEOREM 3.18. $\mathscr{E}$ enjoys the following permanence properties:

(1) $\mathscr{E}$ is closed under group extension.

(2) If $G \in \mathscr{E}, H$ is a t.d.l.c.s.c. group, and $\psi: H \rightarrow G$ is a continuous, injective homomorphism, then $H \in \mathscr{E}$. In particular, $\mathscr{E}$ is closed under taking closed subgroups.

(3) $\mathscr{E}$ is closed under taking quotients by closed normal subgroups.

(4) If $G$ is a residually elementary t.d.l.c.s.c. group, then $G \in \mathscr{E}$. In particular, $\mathscr{E}$ is closed under inverse limits that result in t.d.l.c.s.c. groups.

(5) $\mathscr{E}$ is closed under taking quasi-products.

(6) $\mathscr{E}$ is closed under local direct products.

(7) If $G$ is a t.d.l.c.s.c. group and $\left(C_{i}\right)_{i \in \omega}$ is an $\subseteq$-increasing sequence of elementary subgroups of $G$ such that $N_{G}\left(C_{i}\right)$ is open for each $i$ and $\overline{\bigcup_{i \in \omega} C_{i}}=G$, then $G \in \mathscr{E}$.

\subsection{Elementarily robust classes}

We conclude this section by observing a generalization of our results for elementary groups.

Definition 3.19. Given a class of t.d.l.c.s.c. groups $\mathscr{G}$, the elementary closure of $\mathscr{G}$, denoted $\mathscr{E} \mathscr{G}$, is the smallest class of t.d.l.c.s.c. groups such that

(i) $\mathscr{E} \mathscr{G}$ contains $\mathscr{G}$, all second countable profinite groups, and countable discrete groups.

(ii) $\mathscr{E} \mathscr{G}$ is closed under group extensions of second countable profinite groups, countable discrete groups, and groups in $\mathscr{G}$.

(iii) If $G$ is a t.d.l.c.s.c. group and $G=\bigcup_{i \in \omega} G_{i}$ where $\left(G_{i}\right)_{i \in \omega}$ is an $\subseteq$-increasing sequence of open subgroups of $G$ with $G_{i} \in \mathscr{E} \mathscr{G}$ for each $i$, then $G \in \mathscr{E} \mathscr{G}$. We say $\mathscr{E} \mathscr{G}$ is closed under countable increasing unions. 
The next observation follows from the proof of Theorem 3.18 .

TheOREM 3.20. Suppose $\mathscr{G}$ is a class of t.d.l.c.s.c. groups that is closed under isomorphism of topological groups, taking closed subgroups, and taking Hausdorff quotients. Suppose further $\mathscr{G}$ satisfies the following:

(a) If $H$ is a t.d.l.c.s.c. group and $\psi: H \rightarrow G$ is a continuous, injective homomorphism with $G \in \mathscr{G}$, then $H \in \mathscr{E} \mathscr{G}$.

(b) If $G$ is a t.d.l.c.s.c. group, $H, L \unlhd G,[H, L]=\{1\}$, and $H \in \mathscr{G}$, then $\overline{H L} / L \in \mathscr{E} \mathscr{G}$.

Then the permanence properties stated in Theorem 3.18 hold of $\mathscr{E} \mathscr{G}$.

Classes $\mathscr{G}$ that are closed under isomorphism of topological groups, taking closed subgroups, and taking Hausdorff quotients and, additionally, satisfy $(a)$ and $(b)$ are called elementarily robust. In [29, Theorem 6.3], we show $\mathscr{P}$, the collection of all l.c.s.c. $p$-adic Lie groups for all primes $p$, is elementarily robust.

\section{A characterization of elementary groups}

Our work above, while showing the class of elementary groups is robust, does not give an easy way to identify elementary groups. We here characterize elementary groups in terms of well-founded descriptive-set-theoretic trees giving a criterion by which to identify elementary groups. As a consequence of this characterization, we obtain a new rank on elementary groups.

\subsection{Preliminaries}

Definition 4.1. A topological space $X$ is Polish if the topology is separable and completely metrizable.

By classical results, [18, (5.3)], t.d.l.c.s.c. groups are Polish; i.e. as topological spaces these groups are Polish spaces.

For a Polish space $X, F(X)$ denotes the set of all closed subsets of $X$. This is the so-called Effros Borel space; we remark, but do not use, that $F(X)$ comes with a canonical Borel sigma algebra [18, 12.C].

Theorem 4.2 (Kuratowski, Ryll-Nardzewski [18, (12.13)]). Let $X$ be a Polish space. There is a sequence of Borel functions $d_{n}: F(X) \rightarrow X$ such that for nonempty $F \in F(X),\left\{d_{n}(F)\right\}_{n \in \omega}$ is dense in $F$.

The functions $d_{n}$ are called selector functions for $F(X)$. Given $Y$ a closed subset of $X$, we have that $F(Y) \subseteq F(X)$. A set of selector functions $D$ for $F(X)$ then restricts to a set of selector functions for $F(Y)$. We will abuse notion and say $D$ is also a set of selector functions for $F(Y)$.

We will also require the notion of a descriptive-set-theoretic tree; this notion of a tree differs from the usual graph-theoretic definition. The definitions given here are restricted to the collection of finite sequences of natural numbers. See [18, 2.A] for an excellent, general account.

Denote the collection of finite sequences of natural numbers by $\mathbb{N}<\mathbb{N}$. For sequences $s:=$ $\left(s_{0}, \ldots, s_{n}\right) \in \mathbb{N}<\mathbb{N}$ and $r:=\left(r_{0}, \ldots, r_{m}\right) \in \mathbb{N}<\mathbb{N}$, we write $s \subseteq r$ if $s$ is an initial segment of $r$. That is to say, $n \leqslant m$, and $s_{i}=r_{i}$ for $0 \leqslant i \leqslant n$. The empty sequence, denoted $\emptyset$, is considered 
to be an element of $\mathbb{N}<\mathbb{N}$ and is an initial segment of any $t \in \mathbb{N}<\mathbb{N}$. We define

$$
s^{\wedge} r:=\left(s_{0}, \ldots, s_{n}, r_{0}, \ldots, r_{m}\right) \text {. }
$$

For $t=\left(t_{0}, \ldots, t_{k}\right) \in \mathbb{N}<\mathbb{N}$, the length of $t$, denoted $|t|$, is the number of coordinates; i.e. $|t|:=k+1$. If $|t|=1$, we write $t$ as a natural number as opposed to a sequence of length one. For $0 \leqslant i \leqslant|t|-1, t(i):=t_{i}$. For $\alpha \in \mathbb{N}^{\mathbb{N}}$, we set $\alpha \uparrow_{n}:=(\alpha(0), \ldots, \alpha(n-1))$, so $\alpha \uparrow_{n} \in \mathbb{N}<\mathbb{N}$ for any $n \geqslant 0$.

Definition 4.3. $T \subseteq \mathbb{N}<\mathbb{N}$ is a tree if it is closed under taking initial segments. We call the elements of $T$ the nodes of $T$. If $s \in T$ and there is no $n \in \mathbb{N}$ such that $s^{\frown} n \in T$, we say $s$ is a terminal node of $T$. An infinite branch of $T$ is a sequence $\alpha \in \mathbb{N}^{\mathbb{N}}$ such that $\alpha{ }_{n} \in T$ for all $n$. If $T$ has no infinite branches, we say $T$ is well-founded.

For $T$ a tree and $s \in T$, we put $T_{s}:=\left\{r \in \mathbb{N}<\mathbb{N} \mid s^{\wedge} r \in T\right\}$. The set $T_{s}$ is the tree obtained by taking the elements in $T$ that extend $s$ and deleting the initial segment $s$ from each.

For $T$ a well-founded tree, there is an ordinal valued rank $\rho_{T}$ on the nodes of $T$ defined inductively as follows: If $s \in T$ is terminal, $\rho_{T}(s)=0$. For a non-terminal node $s$,

$$
\rho_{T}(s):=\sup \left\{\rho_{T}\left(s^{\frown} n\right)+1 \mid n \in \mathbb{N} \text { and } s^{\frown} n \in T\right\} .
$$

The rank of a well-founded tree $T$ is then defined to be

$$
\rho(T):=\sup \left\{\rho_{T}(s)+1 \mid s \in T\right\} .
$$

In the case $T \neq \emptyset$, it is easy to verify $\rho(T)=\rho_{T}(\emptyset)+1$. We thus see that $\rho(T)$ is always either a successor ordinal or zero. We make two further observations.

Observation 4.4. Suppose $T \subseteq \mathbb{N}<\mathbb{N}$ is a well-founded tree and $s \in T$. Then

(1) $\rho_{T}(s)+1=\rho\left(T_{s}\right)$ and

(2) $\rho(T)=\sup \left\{\rho\left(T_{i}\right) \mid i \in T\right\}+1$.

\subsection{Decomposition trees}

Fix $G$ a t.d.l.c.s.c. group, $U \in \mathcal{U}(G)$, and $D:=\left\{d_{n}\right\}_{n \in \mathbb{N}}$ selector functions for $F(G)$. For $H \leqslant G$ and $n \in \mathbb{N}$, define

$$
R_{n}^{(U, D)}(H):=\left\langle U \cap H, d_{0}(H), \ldots, d_{n}(H)\right\rangle
$$

where the $d_{i}$ are selector functions from $D$. We now define a tree $T_{(U, D)}(G)$ and associated subgroups of $G$. Put

- $\emptyset \in T_{(U, D)}(G)$ and $G_{\emptyset}:=G$.

- Suppose we have defined $s \in T_{(U, D)}(G)$ and $G_{s} \leqslant G$. Put $s^{\frown} n \in T_{(U, D)}(G)$ and

$$
G_{s^{\urcorner} n}:=\operatorname{Res}\left(R_{n}^{(U, D)}\left(G_{s}\right)\right)
$$

if and only if $G_{s} \neq\{1\}$.

We call $T_{(U, D)}(G)$ the decomposition tree of $G$ with respect to $U$ and $D$. This tree is always non-empty, and any terminal node corresponds to the trivial group. We make one further observation; the proof is straightforward and, therefore, omitted.

Observation 4.5. For any $s \in T_{(U, D)}(G), T_{(U, D)}(G)_{s}=T_{\left(G_{s} \cap U, D\right)}\left(G_{s}\right)$. Further, for $r \in$ $T_{\left(G_{s} \cap U, D\right)}\left(G_{s}\right)$, the associated subgroup $\left(G_{s}\right)_{r}$ is the same as the subgroup $G_{s^{\frown} r}$ associated to $s\urcorner r \in T_{(U, D)}(G)$. 
We now characterize elementary groups in terms of decomposition trees. This requires a technical result.

Lemma 4.6. Suppose $G \in \mathscr{E}$ and $T_{(U, D)}(G)$ is the decomposition tree for $G$ with respect to $U \in \mathcal{U}(G)$ and $D$ a set of selector functions for $F(G)$. If $\operatorname{rk}(G) \leqslant \beta+m$ for $\beta$ a limit ordinal and $m \geqslant 1$ a finite ordinal, then $\operatorname{rk}\left(G_{s}\right) \leqslant \beta$ for all $s \in T_{(U, D)}(G)$ with $|s| \geqslant m$.

Proof. We induct on $m$ for a stronger hypothesis: Suppose $H \leqslant G$ and form $T_{(H \cap U, D)}(H)$. If there is $O \leqslant G$ with $H \leqslant O \leqslant G$ so that $\operatorname{rk}(O) \leqslant \beta+m$ with $\beta$ some limit ordinal and $m \geqslant 1$ a finite ordinal, then $\operatorname{rk}\left(H_{s}\right) \leq \beta$ for any $s \in T_{(H \cap U, D)}(H)$ with $|s| \geqslant m$. In view of Corollary 3.9 and the fact that $H_{s\urcorner n} \leqslant H_{s}$, it suffices to show the induction hypothesis for $s$ such that $|s|=m$.

For the base case, suppose $\operatorname{rk}(O) \leqslant \beta+1$ and fix $i \in \mathbb{N}$. We now have two cases: $\beta=0$ and $\beta$ a non-zero limit ordinal. If $\beta$ is a non-zero limit ordinal, then either $\operatorname{rk}(O)<\beta \operatorname{or} \operatorname{rk}(O)=\beta+1$. In the latter case, $O$ is an increasing union of open subgroups of rank strictly less than $\beta$ since $\beta$ is a limit ordinal. In either case, we may thus write $O=\bigcup_{j \in \omega} L_{j}$ with $\left(L_{j}\right)_{j \in \omega}$ an $\subseteq$-increasing sequence of open subgroups with $\operatorname{rk}\left(L_{j}\right)<\beta$ for each $j \in \omega$. Since $R_{i}^{(H \cap U, D)}\left(H_{\emptyset}\right)$ is compactly generated, $R_{i}^{(H \cap U, D)}\left(H_{\emptyset}\right) \leqslant L_{j}$ for some $j$ and, therefore, has rank strictly less than $\beta$ via Corollary 3.9. A second application of Corollary 3.9 now gives that $\operatorname{rk}\left(H_{i}\right)<\beta$.

Suppose $\beta=0$. If $O$ has construction rank zero, then we are done, so we suppose $\operatorname{rk}(O)=1$. If $O$ is an increasing union of open subgroups of rank zero, then $R_{i}^{(H \cap U, D)}\left(H_{\emptyset}\right)$ has rank zero since compactly generated, and therefore, $H_{i}$ has rank zero as required. Suppose $O$ is a group extension of rank zero groups; say $L \unlhd O$ is such that $\operatorname{rk}(L), \operatorname{rk}(O / L)$ are zero. We see here that

$$
H_{i}=\operatorname{Res}\left(R_{i}^{(H \cap U, D)}\left(H_{\emptyset}\right)\right) \leqslant \operatorname{Res}(O) \leqslant L,
$$

hence $\operatorname{rk}\left(H_{i}\right)=0$. We have thus verified the base case.

Now suppose $\operatorname{rk}(O) \leqslant \beta+m+1$ and fix $s \in T_{(H \cap U, D)}(H)$ with $|s|=m+1$. If $\operatorname{rk}(O)$ is given by an increasing union, there is $L \leqslant{ }_{o} O$ such that $R_{s(0)}^{(H \cap U, D)}\left(H_{\emptyset}\right) \leqslant L$ and $\operatorname{rk}(L) \leqslant \beta+m$. Therefore, $H_{s(0)} \leqslant L$ with $\operatorname{rk}(L) \leqslant \beta+m$, so the induction hypothesis implies $\operatorname{rk}\left(\left(H_{s(0)}\right)_{r}\right) \leqslant \beta$ for

$$
r=(s(1), \ldots, s(m)) \in T_{\left(H_{s(0)} \cap U, D\right)}\left(H_{s(0)}\right) .
$$

In view of Observation 4.5 we conclude that $\left(H_{s(0)}\right)_{r}=H_{s}$, so the induction claim holds when $\operatorname{rk}(O)$ is given by an increasing union.

Suppose $\operatorname{rk}(O)$ is given by a group extension; say $L \unlhd O$ is such that $\operatorname{rk}(L) \leqslant \beta+m$ and $O / L$ is either discrete or profinite. Then,

$$
H_{s(0)}=\operatorname{Res}\left(R_{s(0)}^{(H \cap U, D)}\left(H_{\emptyset}\right)\right) \leqslant \operatorname{Res}(O) \leqslant L .
$$

We may now apply the induction hypothesis to $H_{s(0)} \leqslant L \leqslant G$ to conclude that $\operatorname{rk}\left(\left(H_{s(0)}\right)_{r}\right) \leqslant$ $\beta$ for $r=(s(1), \ldots, s(m))$. Observation 4.5 implies $\left(H_{s(0)}\right)_{r}=H_{s}$, and the induction is finished.

The desired lemma is now in hand: $\operatorname{Suppose} \operatorname{rk}(G) \leqslant \beta+m$ for $\beta$ a limit ordinal and $m \geqslant 1$ a finite ordinal. Applying our work above to $G=H=O$, we conclude that $\operatorname{rk}\left(G_{s}\right) \leqslant \beta$ for all $s \in T_{(U, D)}(G)$ with $|s| \geqslant m$.

Theorem 4.7. Suppose $G$ is a t.d.l.c.s.c. group, $U \in \mathcal{U}(G)$, and $D$ is a set of selector functions for $F(G)$. Then $G$ is elementary if and only if $T_{(U, D)}(G)$ is well-founded. 
Proof. Suppose $G \in \mathscr{E}$; we argue by induction on $\operatorname{rk}(G)$ that $T_{(U, D)}(G)$ is well-founded simultaneously for all $G, U$, and $D$. For the base case, $\operatorname{rk}(G)=0, G$ is residually discrete. Each element in $T_{(U, D)}(G)$ therefore has length at most 2 , and $T_{(U, D)}(G)$ is well-founded.

Suppose $\operatorname{rk}(G)=\alpha+1$. We may write $\operatorname{rk}(G)=\beta+m$ for $\beta \leqslant \alpha$ some limit ordinal and $m \geqslant 1$ a finite ordinal. Applying Lemma 4.6 $\operatorname{rk}\left(G_{s}\right) \leqslant \beta$ for all $s \in T_{(U, D)}(G)$ with $|s|=m$. The induction hypothesis now implies $T_{\left(G_{s} \cap U, D\right)}\left(G_{s}\right)$ is well-founded, and from Observation 4.5 . we infer $T_{(U, D)}(G)_{s}$ is well-founded. Since any infinite branch of $T_{(U, D)}(G)$ gives an infinite branch in $T_{(U, D)}(G)_{s}$ for $s$ the initial segment of length $m, T_{(U, D)}(G)$ must be well-founded. This completes the induction, and we conclude the forward implication.

Suppose $T_{(U, D)}(G)$ is well-founded. We induct on $\rho\left(T_{(U, D)}(G)\right)$ simultaneously for all $G$, $U \in \mathcal{U}(G)$, and $D$ selector functions for $F(G)$ for the claim that $G \in \mathscr{E}$. If $\rho\left(T_{(U, D)}(G)\right)=1$, then $G=\{1\}$ and is obviously elementary. Suppose $\rho\left(T_{(U, D)}(G)\right)=\beta+1$. Observation 4.4 and Observation 4.5 imply $\rho\left(T_{\left(G_{i} \cap U, D\right)}\left(G_{i}\right)\right) \leqslant \beta$ for each $i \in \mathbb{N}$, so the induction hypothesis implies $G_{i} \in \mathscr{E}$ for each $i$. Since $R_{i}^{(U, D)}(G) / G_{i}$ is a SIN group, we further have that $R_{i}^{(U, D)}(G) \in \mathscr{E}$ for each $i$, and since the $\left(R_{i}^{(U, D)}(G)\right)_{i \in \mathbb{N}}$ is an $\subseteq$-increasing exhaustion of $G$, we conclude that $G \in \mathscr{E}$. The induction is now finished, and we conclude the reverse implication.

\subsection{The decomposition rank}

The rank of a decomposition tree $T_{(U, D)}(G)$ as a well-founded tree gives another rank on $\mathscr{E}$. To verify this, we need to show the rank is independent of our choice of $U$ and $D$.

Proposition 4.8. Suppose $G \in \mathscr{E}, U \in \mathcal{U}(G)$, and $D$ is a set of selector functions for $F(G)$. Suppose additionally, $H \in \mathscr{E}, W \in \mathcal{U}(H)$, and $C$ is a set of selector functions for $F(H)$. If $\psi: H \rightarrow G$ is a continuous, injective homomorphism, then

$$
\rho\left(T_{(W, C)}(H)\right) \leqslant \rho\left(T_{(U, D)}(G)\right) .
$$

In particular, for $G \in \mathscr{E}, U$ and $V$ in $\mathcal{U}(G)$, and $D$ and $C$ sets of selector functions for $F(G)$, $\rho\left(T_{(U, D)}(G)\right)=\rho\left(T_{(V, C)}(G)\right)$.

Proof. We induct on $\rho\left(T_{(U, D)}(G)\right)$ simultaneously for all $G \in \mathscr{E}, U \in \mathcal{U}(G)$, and $D$ a set of selector functions for $F(G)$. The base case is obvious since $\rho\left(T_{(U, D)}(G)\right)=1$ implies $G=\{1\}$.

Suppose $\rho\left(T_{(U, D)}(G)\right)=\beta+1$. For each $i, R_{i}^{(W, C)}(H)$ is compactly generated, so there is $n(i)$ with $\psi\left(R_{i}^{(W, C)}(H)\right) \leqslant R_{n(i)}^{(U, D)}(G)$. We thus have that

$$
\psi\left(H_{i}\right)=\psi\left(\operatorname{Res}\left(R_{i}^{(W, C)}(H)\right)\right) \leqslant \operatorname{Res}\left(R_{n(i)}^{(U, D)}(G)\right)=: G_{n(i)} .
$$

The map $\psi$ thereby restricts to $\psi: H_{i} \rightarrow G_{n(i)}$, and Observation 4.4 and Observation 4.5 imply

$$
\rho\left(T_{\left(G_{n(i)} \cap U, D\right)}\left(G_{n(i)}\right)\right)=\rho\left(T_{(U, D)}(G)_{i}\right) \leqslant \beta .
$$

Applying the induction hypothesis,

$$
\rho\left(T_{\left(H_{i} \cap W, C\right)}\left(H_{i}\right)\right) \leqslant \rho\left(T_{\left(G_{n(i)} \cap U, D\right)}\left(G_{n(i)}\right)\right) .
$$

Therefore,

$\rho\left(T_{(W, C)}(H)\right)=\sup _{i \in \omega} \rho\left(T_{\left(H_{i} \cap W, C\right)}\left(H_{i}\right)\right)+1 \leqslant \sup _{i \in \omega} \rho\left(T_{\left(G_{n(i)} \cap U, D\right)}\left(G_{n(i)}\right)\right)+1 \leqslant \rho\left(T_{(U, D)}(G)\right)$.

This finishes the induction, and we conclude the proposition. 
REMARK 4.9. The inequality $\sup _{i \in \omega} \rho\left(T_{\left(G_{n(i)} \cap U, D\right)}\left(G_{n(i)}\right)\right)+1 \leqslant \rho\left(T_{(U, D)}(G)\right)$ appearing above is an equality provided $n(i)$ is unbounded as $i \rightarrow \infty$. Indeed, if $n(i)$ is unbounded, then for all $k \in \omega$, there is $i$ so that $k \leq n(i)$. It follows $G_{k} \hookrightarrow G_{n(i)}$, so appealing to Proposition 4.8, $\rho\left(T_{\left(G_{k} \cap U, D\right)}\left(G_{k}\right)\right) \leq \rho\left(T_{\left(G_{n(i)} \cap U, D\right)}\left(G_{n(i)}\right)\right)$. Therefore,

$$
\rho\left(T_{(U, D)}(G)\right)=\sup _{k \in \omega} \rho\left(T_{\left(G_{k} \cap U, D\right)}\left(G_{k}\right)\right)+1 \leq \sup _{i \in \omega} \rho\left(T_{\left(G_{n(i)} \cap U, D\right)}\left(G_{n(i)}\right)\right)+1
$$

implying equality holds.

We define the decomposition rank of $G \in \mathscr{E}$ to be

$$
\xi(G):=\rho\left(T_{(U, D)}(G)\right)
$$

for some $U \in \mathcal{U}(G)$ and $D$ a set of selector functions for $F(G)$. By Proposition 4.8, $\xi(G)$ is independent of the choice of $U$ and $D$.

Applying Proposition 4.8 again, we see the decomposition rank is indeed a group invariant.

Corollary 4.10. If $G \in \mathscr{E}, H$ is a t.d.l.c.s.c. group, and $\psi: H \rightarrow G$ is a continuous, injective homomorphism, then $\xi(H) \leqslant \xi(G)$. In particular, if $G \in \mathscr{E}$ and $G \simeq H$, then $\xi(G)=$ $\xi(H)$.

We note another important feature of the decomposition rank.

ObSERvation 4.11. Suppose $G$ is a non-trivial t.d.l.c.s.c. group and $\left(O_{i}\right)_{i \in \omega}$ is an increasing exhaustion of $G$ by open subgroups. If each $O_{i}$ is residually discrete, then $G \in \mathscr{E}$ with $\xi(G)=2$, and any decomposition tree for $G$ consists of the root along with its children.

We conclude this subsection by showing the decomposition rank may be computed by taking any countable increasing exhaustion by compactly generated open subgroups. This shows we need not find selector functions to compute the decomposition rank.

Lemma 4.12. Suppose $G$ is a non-trivial elementary group. Then,

(1) If $G=\bigcup_{i \in \omega} O_{i}$ with $\left(O_{i}\right)_{i \in \omega}$ an $\subseteq$-increasing sequence of compactly generated open subgroups of $G$, then $\xi(G)=\sup _{i \in \omega} \xi\left(\operatorname{Res}\left(O_{i}\right)\right)+1$. In particular, $\xi(\operatorname{Res}(H))<\xi(G)$ for any compactly generated $H \leqslant G$.

(2) If $G$ is compactly generated, then $\xi(G)=\xi(\operatorname{Res}(G))+1$.

Proof. For (1), fix $U \in \mathcal{U}(G)$ and $D$ selector functions for $F(G)$. For each $i$, there is $n(i)$ such that $O_{i} \leqslant R_{n(i)}^{(U, D)}(G)$ since $O_{i}$ is compactly generated. Therefore,

$$
\operatorname{Res}\left(O_{i}\right) \leqslant \operatorname{Res}\left(R_{n(i)}^{(U, D)}(G)\right)=G_{n(i)},
$$

and Corollary 4.10 implies $\xi\left(\operatorname{Res}\left(O_{i}\right)\right) \leqslant \xi\left(G_{n(i)}\right)$. We conclude that

$$
\sup _{i \in \omega} \xi\left(\operatorname{Res}\left(O_{i}\right)\right)+1 \leqslant \sup _{i \in \omega} \xi\left(G_{i}\right)+1=\xi(G) .
$$

On the other hand, $\left(O_{i}\right)_{i \in \omega}$ is an exhaustion of $G$ by open subgroups, so for each $k$, there is $n(k)$ with $R_{k}^{(U, D)}(G) \leqslant O_{n(k)}$. Therefore, $G_{k} \leqslant \operatorname{Res}\left(O_{n(k)}\right)$, and applying Corollary 4.10 again,

$$
\xi(G)=\sup _{k \in \omega} \xi\left(G_{k}\right)+1 \leqslant \sup _{i \in \omega} \xi\left(\operatorname{Res}\left(O_{i}\right)\right)+1
$$


Hence, $\xi(G)=\sup _{i \in \omega} \xi\left(\operatorname{Res}\left(O_{i}\right)\right)+1$ as required.

For (2), suppose $G$ is a non-trivial compactly generated elementary group and let $\left(O_{i}\right)_{i \in \omega}$ be an $\subseteq$-increasing exhaustion of $G$ by compactly generated open subgroups. Observe that $\left(\operatorname{Res}\left(O_{i}\right)\right)_{i \in \omega}$ also forms an $\subseteq$-increasing sequence, so in view of Corollary 4.10, $\left(\xi\left(\operatorname{Res}\left(O_{i}\right)\right)\right)_{i \in \omega}$ is a monotone increasing sequence of ordinals. Since $G$ is compactly generated, there is $N$ such that $O_{i}=G$ for all $i \geqslant N$, hence $\operatorname{Res}\left(O_{i}\right)=\operatorname{Res}(G)$ for all $i \geqslant N$. We conclude that $\left(\xi\left(\operatorname{Res}\left(O_{i}\right)\right)\right)_{i \in \omega}$ is a monotone increasing sequence eventually equal to $\xi(\operatorname{Res}(G))$, and therefore, $\sup _{i \in \omega} \xi\left(\operatorname{Res}\left(O_{i}\right)\right)=\xi(\operatorname{Res}(G))$. Applying part $(1), \xi(G)=\xi(\operatorname{Res}(G))+1$.

Section [6] applies Lemma 4.12 to compute the decomposition rank of examples; it is worthwhile to look ahead to these examples to gain some intuition for the decomposition rank.

REMARK 4.13. The decomposition rank uses the second countability assumption. Second countability ensures the existence of selector functions, which allow us to build the decomposition trees in a uniform way. The decomposition trees are also countably branching via second countability.

We observe a consequence of Lemma 4.12. This consequence, while obvious from the statement of the lemma, holds because well-founded decomposition trees characterize elementary groups.

Corollary 4.14. If $G$ is non-trivial, compactly generated, and elementary, then $\operatorname{Res}(G) \lessgtr$ $G$.

Non-trivial compactly generated groups $G$ so that $G=\operatorname{Res}(G)$ therefore cannot be elementary; to build intuition, we encourage the reader to consider how this makes the decomposition trees ill-founded. One naturally questions if this is the only barrier to being elementary. More precisely,

Question 4.15. Suppose $G$ is a t.d.l.c.s.c. group so that $\operatorname{Res}(H) \lesseqgtr H$ for any non-trivial compactly generated closed subgroup $H \leqslant G$. Then is $G$ elementary?

\subsection{Properties of the decomposition rank}

We conclude this section by exploring the decomposition rank further. We shall see the decomposition rank is better behaved than the construction rank but, surprisingly, the two ranks are closely related.

We begin with a general lemma regarding SIN groups.

LEMma 4.16. If $G$ is a compactly generated t.d.l.c.s.c. group and $N \unlhd_{c c} G$ is a SIN group, then $G$ is a SIN group.

Proof. Fix $U \in \mathcal{U}(G)$ and form the subgroup $U N$. Since $N$ is a SIN group, we may find $W \in \mathcal{U}(N)$ with $W \leqslant U$ and $W \unlhd N$. On the other hand, $W \leqslant_{o} N \cap U$, so there is $V \unlhd_{o} U$ 
with $V \cap N \leqslant \leqslant_{o} W$. We now have that

$$
V \cap N \leqslant \bigcap_{u n \in U N} u n W n^{-1} u^{-1}=: J
$$

$J \unlhd U N$, and $J \in \mathcal{U}(N)$.

Since $N$ is cocompact in $G, U N$ has finite index, so $N_{G}(J)$ has finite index. Letting $g_{1}, \ldots, g_{n}$ list left coset representatives for $N_{G}(J)$ in $G$, we see that

$$
\bigcap_{g \in G} g J g^{-1}=\bigcap_{i=1}^{n} g_{i} J g_{i}^{-1} .
$$

Defining $K:=\bigcap_{g \in G} g J g^{-1}$, it follows that $K \in \mathcal{U}(N)$.

Passing to $G / K, \pi(N) \unlhd G / K$ is discrete where $\pi: G \rightarrow G / K$ is the usual projection. The subgroup $N$ is compactly generated since cocompact in a compactly generated group, hence the subgroup $\pi(N)$ is finitely generated. It follows that $\pi(N)$ has an open centralizer in $G / K$. Say $Q \leqslant_{0} \pi(U)$ centralizes $\pi(N)$. Clearly, $Q \unlhd Q \pi(N)$, and using that $\pi(N)$ is cocompact in $G / K$, we additionally see that $Q \pi(N)$ has finite index in $G / K$. Just as in the previous paragraph, there is $L \leqslant_{o} Q$ with $L \unlhd G / K$. It now follows that $\pi^{-1}(L)$ is an open normal subgroup of $G$ contained in $U$.

We conclude that inside every compact open subgroup $U$ of $G$, we may find a compact open normal subgroup of $G$. That is to say, $G$ is a SIN group.

Lemma 4.17. If $G$ is a t.d.l.c.s.c. group, $H, L \unlhd G$, and $H \in \mathscr{E}$, then $\overline{H L} / L \in \mathscr{E}$ and $\xi(\overline{H L} / L) \leqslant \xi(H)$.

Proof. We induct on $\xi(H)$ for the lemma. As the base case is obvious, suppose $\xi(H)=\beta+1$. Fix $U \in \mathcal{U}(\overline{H L}), V \in \mathcal{U}(H)$, and $D$ selector functions for $F(H)$. Put

$$
O_{i}:=\left\langle V^{U}, d_{0}(H)^{U}, \ldots, d_{i}(H)^{U}\right\rangle
$$

with $d_{0}, \ldots, d_{i} \in D$.

For each $i$, the set $U O_{i}$ is a compactly generated open subgroup of $\overline{H L}$ since $O_{i}$ is normalized by $U$. Put $L_{i}:=L \cap U O_{i} \unlhd U O_{i}$ and observe that since the discrete residual is a characteristic subgroup, it is also the case that $\operatorname{Res}\left(O_{i}\right) \unlhd U O_{i}$.

Claim. $\operatorname{Res}\left(U O_{i} / L_{i}\right)=\overline{\operatorname{Res}\left(O_{i}\right) L_{i}} / L_{i}$.

Proof of Claim. The group $O_{i} / \operatorname{Res}\left(O_{i}\right)$ is a SIN group, and $O_{i} / \operatorname{Res}\left(O_{i}\right) \unlhd_{c c} U O_{i} / \operatorname{Res}\left(O_{i}\right)$. Lemma 4.16 therefore implies $U O_{i} / \operatorname{Res}\left(O_{i}\right)$ is a SIN group. Now

$$
\left(U O_{i} / L_{i}\right) /\left(\overline{\operatorname{Res}\left(O_{i}\right) L_{i}} / L_{i}\right) \simeq U O_{i} / \overline{\operatorname{Res}\left(O_{i}\right) L_{i}},
$$

and since the group on the right is a quotient of the SIN group $U O_{i} / \operatorname{Res}\left(O_{i}\right)$, we infer that $\left(U O_{i} / L_{i}\right) /\left(\overline{\operatorname{Res}\left(O_{i}\right) L_{i}}\right)$ is residually discrete. Therefore,

$$
\operatorname{Res}\left(U O_{i} / L_{i}\right) \leqslant \overline{\operatorname{Res}\left(O_{i}\right) L_{i}} / L_{i} .
$$

On the other hand, letting $\pi: U O_{i} \rightarrow U O_{i} / L_{i}$ be the usual projection, $\operatorname{Res}\left(O_{i}\right) \leqslant \pi^{-1}(K)$ for any open normal subgroup $K$ of $U O_{i} / L_{i}$. Thus,

$$
\overline{\operatorname{Res}\left(O_{i}\right) L_{i}} / L_{i}=\overline{\pi\left(\operatorname{Res}\left(O_{i}\right)\right)} \leqslant \operatorname{Res}\left(U O_{i} / L_{i}\right),
$$

and we conclude that $\operatorname{Res}\left(U O_{i} / L_{i}\right)=\overline{\operatorname{Res}\left(O_{i}\right) L_{i}} / L_{i}$. 
The group $O_{i}$ is a compactly generated subgroup of $H$, so Lemma 4.12 implies $\xi\left(\operatorname{Res}\left(O_{i}\right)\right) \leqslant$ $\beta$. We may thus apply the claim along with the induction hypothesis to conclude that $\operatorname{Res}\left(U O_{i} / L_{i}\right)=\overline{\operatorname{Res}\left(O_{i}\right) L_{i}} / L_{i} \in \mathscr{E}$ with

$$
\xi\left(\operatorname{Res}\left(U O_{i} / L_{i}\right)\right)=\xi\left(\overline{\operatorname{Res}\left(O_{i}\right) L_{i}} / L_{i}\right) \leqslant \xi\left(\operatorname{Res}\left(O_{i}\right)\right) \leqslant \beta .
$$

The quotient $U O_{i} / L_{i}$ is thus elementary-by-SIN and, therefore, elementary. Furthermore, the discrete residual of $U O_{i} / L_{i}$ has rank at most $\beta$.

We now see that

$$
\overline{H L} / L=\bigcup_{i \in \omega} U O_{i} L / L
$$

with each $U O_{i} L / L \simeq U O_{i} / L_{i}$ an elementary compactly generated open subgroup, so $\overline{H L} / L \in$ $\mathscr{E}$. Furthermore, by the previous paragraph, the decomposition rank of the discrete residual of each $U O_{i} L / L$ is bounded by $\beta$. Lemma 4.12 therefore implies $\xi(\overline{H L} / L) \leqslant \beta+1=\xi(H)$, and the induction is complete.

Theorem 4.18. If $G \in \mathscr{E}$ and $L \unlhd G$, then $\xi(G / L) \leqslant \xi(G)$.

Proof. We induct on $\xi(G)$. As the base case is obvious, suppose $\xi(G)=\beta+1$. Let $\left(O_{i}\right)_{i \in \omega}$ be an $\subseteq$-increasing sequence of compactly generated open subgroups of $G$ with $G=\bigcup_{i \in \omega} O_{i}$ and for each $i$, put $L_{i}:=L \cap O_{i}$. As in the claim in the previous proof, it follows that

$$
\operatorname{Res}\left(O_{i} / L_{i}\right)=\overline{\operatorname{Res}\left(O_{i}\right) L_{i}} / L_{i} \text {. }
$$

In view of Lemma 4.17 we conclude that $O_{i} / L_{i} \in \mathscr{E}$ with

$$
\xi\left(\operatorname{Res}\left(O_{i} / L_{i}\right)\right)=\xi\left(\overline{\operatorname{Res}\left(O_{i}\right) L_{i}} / L_{i}\right) \leqslant \xi\left(\operatorname{Res}\left(O_{i}\right)\right) \leqslant \beta
$$

where the right-most inequality follows from Lemma 4.12

We also have that

$$
G / L=\bigcup_{i \in \omega} O_{i} L / L
$$

with each $O_{i} L / L \simeq O_{i} / L_{i}$ compactly generated, open, and elementary. Thus, $G / L \in \mathscr{E}$, and since $\xi\left(\operatorname{Res}\left(O_{i} / L_{i}\right)\right) \leqslant \beta$ for each $i$, Lemma 4.12 implies $\xi(G / L) \leqslant \beta+1$ completing the induction.

Proposition 4.19. If $G \in \mathscr{E}$, then $\operatorname{rk}(G) \leqslant 3 \xi(G)$ and $\xi(G) \leqslant \operatorname{rk}(G)+2$.

Proof. For the first claim, we induct on $\xi(G)$. The base case, $\xi(G)=1$, implies $G=\{1\}$; hence, $\operatorname{rk}(G)=0$ and the proposition holds. Suppose $\xi(G)=\beta+1$. Let $\left(O_{i}\right)_{i \in \omega}$ be an $\subseteq$ increasing sequence of compactly generated open subgroups of $G$ with $G=\bigcup_{i \in \omega} O_{i}$. By Lemma 4.12. $\xi\left(\operatorname{Res}\left(O_{i}\right)\right) \leqslant \beta$, so the induction hypothesis implies $\operatorname{rk}\left(\operatorname{Res}\left(O_{i}\right)\right) \leqslant 3 \beta$. Since $O_{i} / \operatorname{Res}\left(O_{i}\right)$ is a SIN group, it follows that

$$
\operatorname{rk}\left(O_{i}\right) \leqslant 3 \beta+2 \text {. }
$$

We conclude that $\operatorname{rk}(G) \leqslant 3(\beta+1)=3 \xi(G)$ finishing the induction.

For the second claim, we induct on $\operatorname{rk}(G)$. For the base case, $G$ is either profinite or discrete, so $\xi(G) \leqslant 2$. Suppose $\operatorname{rk}(G)=\alpha+1$. Consider first the case $\operatorname{rk}(G)$ is given by an increasing union. Say $G=\bigcup_{i \in \omega} O_{i}$ with $\left(O_{i}\right)_{i \in \omega}$ an $\subseteq$-increasing sequence of open subgroups such that $\operatorname{rk}\left(O_{i}\right) \leqslant \alpha$ for each $i$; without loss of generality, we may take the $O_{i}$ to be compactly generated. 
By the induction hypothesis, $\xi\left(O_{i}\right) \leqslant \alpha+2$, so Corollary 4.10 implies $\xi\left(\operatorname{Res}\left(O_{i}\right)\right) \leqslant \alpha+2$. Lemma 4.12 now implies $\xi(G) \leqslant \alpha+3$ as required.

Suppose $\operatorname{rk}(G)$ is given by a group extension. Say $H \unlhd G$ is such that $\operatorname{rk}(H) \leqslant \alpha$ and $G / H$ is either compact or discrete. Write $G=\bigcup_{i \in \omega} O_{i}$ with $\left(O_{i}\right)_{i \in \omega}$ an $\subseteq$-increasing sequence of compactly generated open subgroups of $G$. Since $O_{i} / H \cap O_{i}$ is residually discrete, $\operatorname{Res}\left(O_{i}\right) \leqslant$ $H \cap O_{i}$. In view of Corollary 4.10 and the induction hypothesis,

$$
\xi\left(\operatorname{Res}\left(O_{i}\right)\right) \leqslant \xi(H) \leqslant \alpha+2 .
$$

Applying Lemma 4.12, $\xi(G) \leqslant \alpha+3$, and the induction is complete.

As a corollary, we obtain a bound on the construction rank of a quotient.

Corollary 4.20. If $G \in \mathscr{E}$ and $L \unlhd G$, then $\operatorname{rk}(G / L) \leqslant 3(\operatorname{rk}(G)+2)$.

Proof. Via Proposition 4.19 $\operatorname{rk}(G / L) \leqslant 3 \xi(G / L)$. Theorem4.18 implies $3 \xi(G / L) \leqslant 3 \xi(G)$. Applying Proposition 4.19 again, we conclude that $3 \xi(G) \leqslant 3(\operatorname{rk}(G)+2)$ proving the corollary.

REMARK 4.21. While more technical to define, the decomposition rank is the more useful rank. It has better regularity properties and may be computed algorithmically.

\section{A Further Permanence Property}

\subsection{Preliminaries}

Our first preliminary result is well known; we include a proof for completeness.

Proposition 5.1 (Folklore). If $G$ is a t.d.l.c.s.c. group and $g \in G$ has a countable conjugacy class, then $C_{G}(g)$ is open.

Proof. Since $G / C_{G}(g) \leftrightarrow g^{G}$, we see that $\left|G: C_{G}(g)\right|=\aleph_{0}$. Fixing $\left(g_{i}\right)_{i \in \omega}$ coset representatives for $C_{G}(g)$ in $G$, we thus have that

$$
G=\bigcup_{i \in \omega} g_{i} C_{G}(g)
$$

The Baire category theorem now implies there is some $i \in \omega$ for which $g_{i} C_{G}(g)$ is non-meagre, and it follows that $C_{G}(g)$ is non-meagre. As $C_{G}(g)$ is closed, $C_{G}(g)$ has non-empty interior and, therefore, is open.

We require two additional facts from the literature.

Theorem 5.2 ([18, Theorem (9.10)]). Let $G$ and $H$ be topological groups and $\phi: G \rightarrow H$ a homomorphism. If $G$ is Baire, $H$ is separable, and $\phi$ is Baire measurable, then $\phi$ is continuous.

Theorem 5.3 (Bergman, Lenstra [4, Theorem 3]). Let $G$ be a group and $H$ a subgroup. Then the following are equivalent: 
(1) The set of indices $\left\{\left|H: H \cap g H^{-1}\right|: g \in G\right\}$ has a finite upper bound.

(2) There is $K \unlhd G$ such that $K \sim_{c} H$.

\subsection{The permanence property}

Suppose $H$ and $G$ are t.d.l.c.s.c. groups and $\psi: H \rightarrow G$ is an injective, continuous homomorphism such that $\psi(H)$ is normal and dense in $G$.

Lemma 5.4. For each $W \in \mathcal{U}(H), \operatorname{Comm}_{G}(\psi(W))=G$.

Proof. Fix $g \in G$ and let $\phi_{g}: H \rightarrow H$ be defined by $\phi_{g}(h)=\psi^{-1}\left(g \psi(h) g^{-1}\right)$. Since $\phi_{g}$ is a composition of bijective homomorphisms, $\phi_{g}$ is a bijective homomorphism. For $O \subseteq H$ open,

$$
\phi_{g}^{-1}(O)=\psi^{-1}\left(g^{-1} \psi(O) g\right),
$$

is a Borel set since a continuous, injective image of a Borel set is a Borel set [18, (15.1)]. The homomorphism $\phi_{g}$ is thus a Borel map, and applying Theorem5.2, $\phi_{g}$ is continuous. The same argument applied to $\phi_{g^{-1}}=\phi_{g}^{-1}$ gives that $\phi_{g}^{-1}$ is also continuous, hence $\phi_{g}$ is an isomorphism of topological groups.

Fixing $W \in \mathcal{U}(H)$, we now have that $\phi_{g}(W) \in \mathcal{U}(H)$, hence $W \sim_{c} \phi_{g}(W)$. We conclude that

$$
\psi(W) \sim_{c} \psi\left(\phi_{g}(W)\right)=g \psi(W) g^{-1},
$$

and it follows that $\operatorname{Comm}_{G}(\psi(W))=G$.

Lemma 5.5. For each $U \in \mathcal{U}(G)$, there is $W \in \mathcal{U}(H)$ with $\psi(W) \unlhd U$.

Proof. Fix $U \in \mathcal{U}(G)$. Since $\psi$ is continuous, we may find $V \in \mathcal{U}(H)$ such that $\psi(V) \leqslant U$. For each $n \geqslant 1$, put

$$
\Omega_{n}:=\left\{g \in U|| \psi(V): \psi(V) \cap g \psi(V) g^{-1} \mid \leqslant n\right\} .
$$

Claim. For each $n \geqslant 1, \Omega_{n}$ is closed.

Proof of Claim. Suppose $\left(g_{i}\right)_{i \in \omega} \subseteq \Omega_{n}$ converges to $g$. For each $i$, let $k_{1}^{i}, \ldots, k_{n}^{i}$ be coset representatives, with possible repetition, for $\psi(V) \cap g_{i} \psi(V) g_{i}^{-1}$ in $\psi(V)$. We may assume $k_{j}^{i} \rightarrow$ $k_{j}$ as $i \rightarrow \infty$ for each $1 \leqslant j \leqslant n$ by passing to a subsequence.

Consider $x \in \psi(V)$. For each $i$, there is $k_{j}^{i}$ and $y_{i} \in \psi(V) \cap g_{i} \psi(V) g_{i}^{-1}$ with $x=k_{j}^{i} y_{i}$. By passing to a subsequence, we may assume $j=j_{0}$ for all $i$. As $k_{j_{0}}^{i} \rightarrow k_{j_{0}}$, we conclude that $y_{i} \rightarrow y$ with $y \in \psi(V) \cap g \psi(V) g^{-1}$, so $x=k_{j_{0}} y$. It now follows that

$$
\psi(V)=\bigcup_{j=1}^{n} k_{j}\left(\psi(V) \cap g \psi(V) g^{-1}\right),
$$

and $\left|\psi(V): \psi(V) \cap g \psi(V) g^{-1}\right| \leqslant n$. Therefore, $g \in \Omega_{n}$.

Since $U=\bigcup_{n \geqslant 1} \Omega_{n}$, the Baire category theorem implies there is some $n$ such that $\Omega_{n}$ is nonmeagre. In view of the claim, $\Omega_{n}$ has non-empty interior, and it follows there are $u_{1}, \ldots, u_{k} \in U$ with $U=\bigcup_{i=1}^{k} u_{i} \Omega_{n}$. Let $m \geqslant n$ be such that $u_{1}, \ldots, u_{k} \in \Omega_{m}$. We claim $U=\Omega_{m n}$.

For $x \in U$, we may take $x=u_{i} w$ for some $1 \leqslant i \leqslant k$ and $w \in \Omega_{n}$. Since $\mid \psi(V): \psi(V) \cap$ $w \psi(V) w^{-1} \mid \leqslant n$,

$$
\left|\psi(V) \cap u_{i}^{-1} \psi(V) u_{i}: \psi(V) \cap u_{i}^{-1} \psi(V) u_{i} \cap w \psi(V) w^{-1}\right| \leqslant n .
$$


Conjugating with $u_{i}$, we obtain that

$$
\left|\psi(V) \cap u_{i} \psi(V) u_{i}^{-1}: \psi(V) \cap u_{i} \psi(V) u_{i}^{-1} \cap u_{i} w \psi(V) w^{-1} u_{i}^{-1}\right| \leqslant n .
$$

It now follows that $\left|\psi(V): \psi(V) \cap u_{i} \psi(V) u_{i}^{-1} \cap x \psi(V) x^{-1}\right| \leqslant m n$, and we infer that

$$
\left|\psi(V): \psi(V) \cap x \psi(V) x^{-1}\right| \leqslant m n .
$$

Therefore, $U=\Omega_{m n}$.

Theorem 5.3 now gives $K \unlhd U$ with $K \sim_{c} \psi(V)$. Form $\bar{K}$. Certainly, it remains the case that $|\psi(V): \bar{K} \cap \psi(V)|<\infty$. On the other hand, there are $k_{1}, \ldots, k_{n} \in K$ with $K \subseteq \bigcup_{i=1}^{n} k_{i} \psi(V)$. Since the latter set is closed, $\bar{K} \subseteq \bigcup_{i=1}^{n} k_{i} \psi(V)$, so

$$
\bar{K}=\bigcup_{i=1}^{n} k_{i}(\psi(V) \cap \bar{K}) .
$$

Therefore, $|\bar{K}: \bar{K} \cap \psi(V)|<\infty$, and $\bar{K} \sim_{c} \psi(V)$.

Since $\bar{K} \cap \psi(V)$ is open in $\bar{K}$, we may find $L \unlhd_{o} U$ so that $\bar{K} \cap L \leqslant_{o} \bar{K} \cap \psi(V)$. Since $\bar{K} \cap$ $\psi(V)$ is finite index in $\psi(V), \bar{K} \cap L$ is also finite index in $\psi(V)$. The group $W:=\psi^{-1}(\bar{K} \cap L)$ is thus closed and finite index in $V$ and, therefore, is compact and open in $H$. On the other hand, $\psi(W) \unlhd U$, so $W$ satisfies the lemma.

Theorem 5.6. Suppose $H \in \mathscr{E}, G$ is a t.d.l.c.s.c. group, and $\psi: H \rightarrow G$ is an injective, continuous homomorphism with $\psi(H)$ normal and dense in $G$. Then $G \in \mathscr{E}$.

Proof. Fix $U \in \mathcal{U}(G)$ and apply Lemma5.5 to find $W \in \mathcal{U}(H)$ with $\psi(W) \unlhd U$. Lemma 5.4 gives that $\psi(W)$ is commensurated, whereby

$$
Q C_{G}(U / \psi(W)):=\{g \in G \mid \exists V \in \mathcal{U}(G) \text { such that }[g, V \cap U] \subseteq \psi(W)\}
$$

is a normal subgroup of $G$ via Proposition 2.8, We observe that $\psi(H) \cap U \unlhd U$ and $\psi(H) \cap$ $U / \psi(W)$ is countable, so $\psi(H) \cap U / \psi(W)$ is quasi-central in $U / \psi(W)$. It follows that $\psi(H) \cap$ $U \leqslant Q C_{G}(U / \psi(W))$ and, therefore,

$$
\overline{\psi(H) \cap Q C_{G}(U / \psi(W))} \unlhd_{o} G .
$$

To prove the desired theorem, it thus suffices to show $\overline{\psi(H) \cap Q C_{G}(U / \psi(W))} \in \mathscr{E}$, so we may assume, without loss of generality, that $\psi(H) \cap Q C_{G}(U / \psi(W))$ is dense in $G$. We may also assume $G$ is compactly generated since $G$ is an increasing union of compactly generated open subgroups.

We induct on the decomposition rank of $H$ for the theorem. For the base case, $\xi(H)=1$, $H=\{1\}$, and the result is trivial. Suppose $H \in \mathscr{E}$ with $\xi(H)=\beta+1$. By our reductions, $G$ is compactly generated; fix $X$ a compact generating set. Proposition 2.4 gives a finite symmetric $A \subseteq G$ containing 1 so that $X \subseteq A U$ and $U A U=A U$. We may take

$$
A \subseteq \psi(H) \cap Q C_{G}(U / \psi(W))
$$

since $\psi(H) \cap Q C_{G}(U / \psi(W))$ is dense in $G$. Applying Proposition 2.4 again, we have that $G=\langle A\rangle U$.

Put $B:=\{\psi(W) a \mid a \in A\}$.

Claim. $N_{G}(B)$ is open.

Proof of Claim. Since $A \subseteq Q C_{G}(U / \psi(W))$ and is finite, there is $V \leqslant_{o} U$ such that $[a, V] \subseteq$ $\psi(W)$ for each $a \in A$. Taking $v \in V$ and $a \in A$, we have that

$$
v \psi(W) a v^{-1}=\psi(W) v a v^{-1}
$$


and since $a v a^{-1} v^{-1}=[a, v] \in \psi(W), \psi(W) v a v^{-1}=\psi(W) a$. Hence, $V \leqslant N_{G}(B)$, and $N_{G}(B)$ is open.

In view of the claim, $\langle B\rangle \unlhd\langle B\rangle V \leqslant_{o} G$ where $V$ is as in the proof of the claim, so

$$
\langle B\rangle \unlhd \overline{\langle B\rangle} \unlhd_{c c}\langle B\rangle V .
$$

Since $\psi^{-1}(B)$ is a compact open subset of $H, L:=\psi^{-1}(\langle B\rangle)$ is a compactly generated open subgroup of $H$. Lemma 4.12 implies $\xi(\operatorname{Res}(L)) \leqslant \beta$. As in the proof of Lemma 5.4. for $g \in\langle B\rangle V$, the map $\phi_{g}: L \rightarrow L$ defined by $\phi_{g}(l):=\psi^{-1}\left(g \psi(l) g^{-1}\right)$ is a topological group automorphism of $L$, hence $\phi_{g}(\operatorname{Res}(L))=\operatorname{Res}(L)$. We conclude that

$$
g \psi(\operatorname{Res}(L)) g^{-1}=\psi\left(\phi_{g}(\operatorname{Res}(L))\right)=\psi(\operatorname{Res}(L)),
$$

and therefore, $\psi(\operatorname{Res}(L)) \unlhd\langle B\rangle V$. The group $\psi(\operatorname{Res}(L))$ is thus normal and dense in $\overline{\psi(\operatorname{Res}(L))}$, and $\xi(\operatorname{Res}(L)) \leqslant \beta$. The induction hypothesis thus implies $R:=\overline{\psi(\operatorname{Res}(L))} \in \mathscr{E}$.

The map $\psi$ induces an injective, continuous homomorphism

$$
\chi: L / \psi^{-1}(R) \rightarrow \overline{\langle B\rangle} / R
$$

with dense, normal image. Since a quotient of the $\operatorname{SIN}$ group $L / \operatorname{Res}(L)$, the group $L / \psi^{-1}(R)$ is a SIN group. Let $K \unlhd L / \psi^{-1}(R)$ be a compact open normal subgroup. We now have that $\chi(K) \unlhd\langle B\rangle R / R$ and since $\chi(K)$ is closed, $\chi(K) \unlhd \overline{\langle B\rangle} / R$. The image of $L / \psi^{-1}(R)$ in $\overline{\langle B\rangle} / R / \chi(K)$ is then dense, normal, and countable. Hence, $Q Z(\overline{\langle B\rangle} / R / \chi(K))$ is dense in $\overline{\langle B\rangle} / R / \chi(K)$, and

$$
\operatorname{SIN}(\overline{\langle B\rangle} / R / \chi(K))=\overline{\langle B\rangle} / R / \chi(K) \text {. }
$$

In view of Theorem 3.18 , it follows that $\overline{\langle B\rangle} / R \in \mathscr{E}$ and further, that $\langle B\rangle V \in \mathscr{E}$.

By choice of $B$ and $V,\langle B\rangle V$ is a finite index subgroup of $G=\langle A\rangle U$. Theorem 3.18 implies the normal core of $\langle B\rangle V$ is elementary, whereby $G$ is elementary-by-finite and, thus, elementary. This completes the induction, and we conclude the theorem.

\section{Examples of elementary groups}

\subsection{First examples and non-examples}

Proposition 6.1. The following are elementary groups:

(1) T.d.l.c.s.c. SIN groups; in particular, abelian t.d.l.c.s.c. groups.

(2) T.d.l.c.s.c. solvable groups.

(3) Locally elliptic t.d.l.c.s.c. groups.

(4) T.d.l.c.s.c. groups containing a compact open subgroup that has a dense quasi-centre. In particular, any t.d.l.c.s.c. group that contains $F^{\mathbb{N}}$ as a compact open subgroup for $F$ some finite group.

Proof. (1) and (2) follow immediately since SIN groups are profinite-by-discrete and solvable groups are built via group extension from abelian groups. (3) follows since locally elliptic t.d.l.c.s.c. groups are countable increasing unions of profinite groups.

For (4), suppose $G$ is a t.d.l.c.s.c. group and $U \in \mathcal{U}(G)$ has a dense quasi-centre. Since $Q Z(U) \leqslant Q Z(G) \leqslant \operatorname{SIN}(G)$, the $\operatorname{SIN}$-core $\operatorname{SIN}(G)$ is open in $G$. Appealing to Proposition 2.14, $\operatorname{SIN}(G)$ is a countable increasing union of $\operatorname{SIN}$ groups, so $\operatorname{SIN}(G)$ is also elementary. It now follows that $G$ is elementary. 
It is illuminating to compute the construction and decomposition ranks in the first two examples. Suppose $G$ is a $S I N$ group. Since $G$ admits compact open normal subgroups, $G$ is compact-by-discrete, hence $\operatorname{rk}(G) \leqslant 1$. For the decomposition rank, if $G=\{1\}$, then $\xi(G)=1$. For $G$ non-trivial, Observation 4.11 implies $\xi(G)=2$ since $G$ is residually discrete.

Things are more interesting for solvable groups. Suppose $G$ is $n \geqslant 1$-step solvable. That is to say the $n$-th term of the closed derived series is trivial; see Section 8 for a precise definition.

Claim. $\operatorname{rk}(G) \leqslant 2 n-1$.

Proof of Claim. We argue by induction on $n$. For the base case, $G$ is abelian and the above paragraph gives that $\operatorname{rk}(G) \leqslant 1$. Suppose $G$ is $(n+1)$-step solvable. By the induction hypothesis, $\operatorname{rk}(\overline{[G, G]}) \leqslant 2 n-1$. On the other hand, let $\pi: G \rightarrow G / \overline{[G, G]}$ be the usual projection. Since $G / \overline{[G, G]}$ is abelian, there is $K \unlhd G / \overline{[G, G]}$ compact and open, so $\pi^{-1}(K)$ is an extension of a compact group by an at most construction rank $2 n-1$ group. We infer that $\operatorname{rk}\left(\pi^{-1}(K)\right) \leqslant 2 n$. Since $G / \pi^{-1}(K)$ is discrete, we conclude that $\operatorname{rk}(G) \leqslant 2 n+1$ completing the induction.

We now compute an upper bound for the decomposition rank.

Claim. $\quad \xi(G) \leqslant n+1$.

Proof of Claim. We argue by induction on $n$. The base case we have since SIN groups are residually discrete. Suppose $G$ is $(n+1)$-step solvable. Write $G$ as an increasing union of compactly generated open subgroups $O_{n}$. Since $G / \overline{[G, G]}$ is residually discrete, $\operatorname{Res}\left(O_{n}\right) \leqslant$ $\overline{[G, G]}$ for each $n$, and via the induction hypothesis and Corollary 4.10, $\xi\left(\operatorname{Res}\left(O_{n}\right)\right) \leqslant n+1$. Lemma 4.12 now implies

$$
\xi(G)=\sup _{n \in \omega} \xi\left(\operatorname{Res}\left(O_{n}\right)\right)+1 \leqslant n+2
$$

completing the induction.

Of course, we only computed upper bounds on the ranks in the above examples. Lower bounds require knowing more about the specific structure. For example, any of the groups above can be discrete; in such a case, either rank is at most two. We compute lower bounds later in this section. We shall see the decomposition rank is preferred for computing lower bounds.

REMARK 6.2. We have an algorithm for computing the decomposition rank: Take an exhaustion by compactly generated open subgroups; compute the decomposition rank of the discrete residual of each term of the exhaustion; and take the supremum of the ranks plus one. The construction rank seems to be, somewhat at odds with the name, non-constructive to compute. There does not seem to be a way to identify whether or not the rank is given by an increasing union or by a group extension. Complicating things further, in the case the construction rank is given by group extension, it is not clear how to compute the correct normal subgroup that witnesses the construction rank.

We now consider non-examples. Recall there are many non-discrete compactly generated t.d.l.c.s.c. groups that are topologically simple. For example, let $\mathcal{T}_{n}$ denote the $n$-regular tree for any $n \geqslant 3$. By work of J. Tits [26, there is an index two non-discrete compactly generated t.d.l.c.s.c. subgroup of $\operatorname{Aut}\left(\mathcal{T}_{n}\right)$, denoted $A u t^{+}\left(\mathcal{T}_{n}\right)$, that is topologically simple. Alternatively, 
$P S L_{n}\left(\mathbb{Q}_{p}\right)$ for $n \geqslant 3$ is a non-discrete compactly generated topologically simple t.d.l.c.s.c. group; cf. [3] [12].

Proposition 6.3. If a t.d.l.c.s.c. group $G$ is compactly generated, topologically simple, and elementary, then $G$ is discrete. In particular, $P S L_{n}\left(\mathbb{Q}_{p}\right)$ and $A u t^{+}\left(\mathcal{T}_{n}\right)$ for $n \geqslant 3$ are non-elementary.

Proof. If $G$ is trivial, we are done. Else $\operatorname{Res}(G) \lesseqgtr G$ via Corollary 4.14, Topological simplicity implies $\operatorname{Res}(G)=\{1\}$, and it follows that $\{1\}$ is open. Therefore, $G$ is discrete.

REMARK 6.4. Although Proposition 6.3 shows there are no non-discrete compactly generated topologically simple groups in $\mathscr{E}$, there are non-discrete non-compactly generated topologically simple groups in $\mathscr{E}$. For example, the topologically simple groups built by Willis [30. Proposition 3.4] are such groups. The construction ranks of Willis' examples are necessarily given by increasing union. These examples are indeed increasing unions of compact groups and as a corollary, have construction rank one and decomposition rank two.

\subsection{A family of elementary groups with decomposition rank unbounded below $\omega$}

We construct a family of elementary groups with members of arbitrarily large finite decomposition rank. It will then follow via Proposition 4.19 that there are members with arbitrarily large finite construction rank. For this construction some notation is required: For $K \leqslant L$, we use $\langle\langle K\rangle\rangle_{L}$ to denote the normal subgroup generated by $K$ in $L$. When clear from context, we drop the subscript.

Let $A_{5}$ be the alternating group on five letters; recall $A_{5}$ is a non-abelian finite simple group. Let $S$ denote the infinite four generated simple group built by G. Higman 17. Form $H:=S^{5} \rtimes A_{5}$ where $A_{5} \curvearrowright\{0,1,2,3,4\}$ in the usual fashion and fix a transitive, free action of $H$ on $\mathbb{N}$. Observe that the normal subgroup generated by $A:=A_{5}$ in $H$ is $H$ itself.

We inductively define compactly generated elementary groups $L_{n}$ and distinguish $K_{n} \in$ $\mathcal{U}\left(L_{n}\right)$ so that $\left\langle\left\langle K_{n}\right\rangle\right\rangle=L_{n}$. For the base case, $n=1$, define $L_{1}:=H$ and $K_{1}:=A$. So $L_{1}$ is compactly generated, $K_{1}$ is a compact open subgroup of $L_{1}$, and $\left\langle\left\langle K_{1}\right\rangle\right\rangle=L_{1}$. Suppose we have defined a compactly generated $L_{n}$ with a compact open subgroup $K_{n}$ so that $\left\langle\left\langle K_{n}\right\rangle\right\rangle=L_{n}$. Let $\left(L_{n}^{i}\right)_{i \in \mathbb{N}}$ and $\left(K_{n}^{i}\right)_{i \in \mathbb{N}}$ list countably many copies of $L_{n}$ and $K_{n}$, respectively, and form $\bigoplus_{i \in \mathbb{N}}\left(L_{n}^{i}, K_{n}^{i}\right)$. Taking the previously fixed action of $H$ on $\mathbb{N}$, we see that $H \curvearrowright \bigoplus_{i \in \mathbb{N}}\left(L_{n}^{i}, K_{n}^{i}\right)$ by shift; see the discussion after Definition 2.3. We may thus form

$$
L_{n+1}:=\bigoplus_{i \in \mathbb{N}}\left(L_{n}^{i}, K_{n}^{i}\right) \rtimes H
$$

and put $K_{n+1}:=K_{n}^{\mathbb{N}} \rtimes A$. Certainly, $K_{n+1}$ is a compact open subgroup of $L_{n+1}$. Letting $X$ be a compact generating set for $L_{n}^{0}$ and $F$ be a finite generating set for $H$ in $L_{n+1}$, one verifies that $X \times \prod_{i>0} K_{n}^{i} \cup F$ is a compact generating set for $L_{n+1}$. It is easy to further verify that $\left\langle\left\langle K_{n+1}\right\rangle\right\rangle_{L_{n+1}}=L_{n+1}$. This completes our inductive construction.

Proposition 6.5. For each $n \geqslant 1, \xi\left(L_{n}\right) \geqslant n+1$.

Proof. We argue by induction on $n$. For the base case, $L_{1}=H$ is non-trivial and discrete. A fortiori, $L_{1}$ is residually discrete, hence $\xi\left(L_{1}\right)=2$ via Observation 4.11 , 
Suppose the induction hypothesis holds up to $n$ and consider $L_{n+1}$. We first compute $\operatorname{Res}\left(L_{n+1}\right)$. Consider $O \unlhd_{o} L_{n+1}$. Since $K_{n}^{\mathbb{N}}$ is a compact open subgroup of $L_{n+1}, O$ must contain

$$
K_{n}^{(k, \infty)}:=\left\{f: \mathbb{N} \rightarrow K_{n} \mid f(0)=\cdots=f(k)=1\right\}
$$

for some $k \in \mathbb{N}$. Since $H$ acts transitively on $\mathbb{N}$ and $O$ is normal, $O$ indeed contains $K_{n}^{\mathbb{N}}$. Recalling $\left\langle\left\langle K_{n}\right\rangle\right\rangle_{L_{n}}=L_{n}$, we conclude that

$$
\bigoplus_{i \in \mathbb{N}}\left(L_{n}^{i}, K_{n}^{i}\right)=\left\langle\left\langle K_{n}^{\mathbb{N}}\right\rangle\right\rangle_{L_{n+1}} \leqslant O
$$

It now follows that $\operatorname{Res}\left(L_{n+1}\right)=\bigoplus_{i \in \mathbb{N}}\left(L_{n}^{i}, K_{n}^{i}\right)$.

Lemma 4.12 gives that $\xi\left(L_{n+1}\right)=\xi\left(\operatorname{Res}\left(L_{n+1}\right)\right)+1$ because $L_{n+1}$ is compactly generated. The group $L_{n}$ admits a continuous injection into $\operatorname{Res}\left(L_{n+1}\right)$, so

$$
\xi\left(\operatorname{Res}\left(L_{n+1}\right)\right) \geqslant \xi\left(L_{n}\right) \geqslant n+1
$$

via Corollary 4.10 and the induction hypothesis. We conclude that $\xi\left(L_{n+1}\right) \geqslant n+2$, and the induction is complete.

The set $\left\{L_{n} \mid n \geqslant 1\right\}$ is thus a family of elementary groups with members of arbitrarily large finite decomposition rank. In view of Proposition 4.19, we infer that $\operatorname{rk}\left(L_{n}\right) \geqslant n-1$ for each $n$, hence $\left\{L_{n} \mid n \geqslant 1\right\}$ is also a family of elementary groups with members of arbitrarily large finite construction rank.

Corollary 6.6. For $G:=\bigoplus_{n \geqslant 1}\left(L_{n}, K_{n}\right), \xi(G)=\omega+1$. It follows that $\operatorname{rk}(G)=\omega+1$.

Proof. For each $n \geqslant 1$, there is a continuous injection $L_{n} \hookrightarrow G$. Via Proposition 6.5 and Corollary 4.10, $n+1 \leqslant \xi(G)$ for all $n \geqslant 1$, so $\omega \leqslant \xi(G)$. Since the decomposition rank is always a successor ordinal or zero, we conclude that $\omega+1 \leqslant \xi(G)$. The converse inequality is an easy exercise.

The decomposition rank arises from well-founded trees $T \subseteq \mathbb{N}^{<\mathbb{N}}$, hence it is always less than $\omega_{1}$, the first uncountable ordinal. However, it is unknown if $\omega_{1}$ is the least upper bound.

QUESTION 6.7. Are there elementary groups of arbitrarily large decomposition rank below $\omega_{1}$ ?

REMARK 6.8. Results for elementary amenable groups suggest a positive answer to the question; cf. 22. Alternatively, using bi-infinite iterated wreath products similar to M.G. Brin's construction in [5], one can build elementary groups of decomposition rank $\omega+2$. The examples of decomposition rank $\omega+2$ are compelling as they are compactly generated elementary groups with transfinite rank. It seems plausible, albeit difficult, this construction can be iterated to build groups of arbitrarily large rank below $\omega_{1}$. The decomposition rank $\omega+2$ examples will appear in a forthcoming paper joint with Colin Reid.

An answer in either direction to the question would be quite interesting. A negative answer would place strong restrictions on the class of elementary groups. A positive answer would imply there is no $S Q$-universal elementary group for the class of elementary groups. This would in particular imply there is no surjectively universal t.d.l.c.s.c. group for the class of t.d.l.c.s.c. groups answering a question of S. Gao and M. Xuan $1 \mathbf{1 3}$. 


\section{Application 1: Structure theorems}

For our first application, we consider elementary groups appearing as normal subgroups or as quotients of an arbitrary t.d.l.c.s.c. group to arrive at general structure theorems.

\subsection{Preliminaries}

We require basic graph-theoretic notions. For a graph $\Gamma, V \Gamma$ denotes the vertices of $\Gamma$, and $E \Gamma$ denotes the edges. We take as a convention that edges connect distinct vertices. If $w \in V \Gamma$ is connected to $v \in V \Gamma$ by an edge, we say $w$ is a neighbour of $v$. In the case $\Gamma$ is a vertex transitive locally finite graph, the degree of $\Gamma$, denoted $\operatorname{deg}(\Gamma)$, is the number of neighbors of some (any) $v \in V \Gamma$. When a graph is connected, there is a metric given by the least length of an edge path.

Graphs play an important role in the study of t.d.l.c. groups via an old result of H. Abels.

TheOREM 7.1 (Abels [1). Let $G$ be a compactly generated t.d.l.c. group. Then there is a locally finite connected graph $\Gamma$ on which $G$ acts continuously and vertex transitively by graph automorphisms such that for all $v \in V \Gamma$, the stabilizer of $v$ in $G$ is compact and open.

For $G$ a t.d.l.c. group, a connected locally finite graph on which $G$ acts continuously and vertex transitively by graph automorphisms with compact open stabilizers is called a Cayley-Abels graph for $G$. Theorem 7.1 shows every compactly generated t.d.l.c. group admits a Cayley-Abels graph; the converse also holds. We make one further remark: If $G$ is a compactly generated t.d.l.c. group and $\Gamma$ is a Cayley-Abels graph for $G$, then the kernel of $G \curvearrowright \Gamma$ is a compact normal subgroup. Therefore, in the case $G$ has no non-trivial compact normal subgroups, $G \curvearrowright \Gamma$ faithfully. We direct the reader to [19] for a pleasant, self contained discussion of the Cayley-Abels graph; in 19, the Cayley-Abels graph is called the rough Cayley graph.

Cayley-Abels graphs give rise to a finite invariant: For a compactly generated t.d.l.c.s.c. group $G$, the degree of $G$ is

$$
\operatorname{deg}(G):=\min \{\operatorname{deg}(\Gamma) \mid \Gamma \text { is a Cayley-Abels graph for } G\} .
$$

We shall see this invariant plays an important role in the structure theory.

If $G$ is a group and $X$ a set with a $G$-action, then a $G$-congruence $\sigma$ on $X$ is an equivalence relation on $X$ such that for all $g \in G, x \sim_{\sigma} y$ if and only if $g . x \sim_{\sigma} g . y$. The equivalence classes of $\sigma$ are called the blocks of $\sigma$. For $x \in X$, the block containing $x$ is denoted $x^{\sigma}$.

We make use of $G$-congruences on Cayley-Abels graphs. In particular, suppose $G$ is a compactly generated t.d.l.c. group with $H \unlhd G$ and suppose $\Gamma$ is a Cayley-Abels graph for $G$. The orbits of $H$ on $V \Gamma$ induce a $G$-congruence on $V \Gamma$; call this $G$-congruence $\sigma$. We may now define a quotient graph $\Gamma / \sigma$ by $V \Gamma / \sigma:=\left\{v^{\sigma} \mid v \in V \Gamma\right\}$ and

$$
E \Gamma / \sigma:=\left\{\left\{v^{\sigma}, w^{\sigma}\right\} \mid v^{\sigma} \neq w^{\sigma} \text { and } \exists v^{\prime} \in v^{\sigma} w^{\prime} \in w^{\sigma} \text { such that }\{v, w\} \in E \Gamma\right\}
$$

This quotient graph has two very useful properties; the proofs are left to the reader.

OBSERVATION 7.2 .

(1) $\Gamma / \sigma$ is connected, locally finite, vertex transitive, and $\operatorname{deg}(\Gamma / \sigma) \leqslant \operatorname{deg}(\Gamma)$.

(2) $G / H \curvearrowright \Gamma / \sigma$ continuously and transitively by graph automorphisms. Additionally, for each $v^{\sigma} \in V \Gamma / \sigma$, the stabilizer of $v^{\sigma}$ is compact and open in $G / H$.

We lastly require a powerful theorem of Caprace and Monod. 
Theorem 7.3 (Caprace, Monod [8, Theorem B]). Let $G$ be a compactly generated t.d.l.c. group. Then one of the following holds:

(1) $G$ has an infinite discrete normal subgroup.

(2) $G$ has a non-trivial locally elliptic radical.

(3) $G$ has exactly $0<n<\infty$ many minimal non-trivial closed normal subgroups.

REMARK 7.4. The statement of alternative (2) in Theorem 7.3 is weaker than the statement made in $[\mathbf{8}$, Theorem B] that $G$ has a non-trivial compact normal subgroup. Examples show the stronger claim is false, however; the error occurs in the proof of $[\mathbf{8}$, Proposition 2.6] on which [8, Theorem B] relies. Fortunately, upon replacing "compact normal subgroup" by "locally elliptic normal subgroup" in the statement of [8, Proposition 2.6], it is an easy exercise to fix the proof given by Caprace and Monod and thus to prove Theorem 7.3 . The author has been informed that Caprace and Monod are preparing a correction which implies Theorem 7.3 .

\subsection{The elementary radical and the elementary residual}

Let $G$ be a t.d.l.c.s.c. group and put

$$
\mathcal{S}_{\mathscr{E}}(G):=\{N \unlhd G \mid N \text { is elementary }\} .
$$

We claim $\subseteq$-chains in $\mathcal{S}_{\mathscr{E}}(G)$ admit upper bounds. Indeed, suppose $\left(N_{\alpha}\right)_{\alpha<\lambda}$ is an $\subseteq$-increasing chain in $\mathcal{S}_{\mathscr{E}}(G)$. Fix $U \in \mathcal{U}(G)$ and consider $\left(U N_{\alpha}\right)_{\alpha<\lambda}$. Since $\bigcup_{\alpha<\lambda} U N_{\alpha}$ is open in $G$, it is a Lindelöf space when considered as a subspace, so there is a countable subcover $\left(U N_{\alpha_{i}}\right)_{i \in \omega}$. Each $U N_{\alpha_{i}}$ is elementary, and therefore, $\bigcup_{i \in \omega} U N_{\alpha_{i}} \in \mathscr{E}$. Applying Theorem $3.18 . \overline{\bigcup_{\alpha<\lambda} N_{\alpha}} \in \mathscr{E}$. Since $\overline{\bigcup_{\alpha<\lambda} N_{\alpha}} \unlhd G$, we conclude that $\overline{\bigcup_{\alpha<\lambda} N_{\alpha}} \in \mathcal{S}_{\mathscr{E}}(G)$. Inclusion chains in $\mathcal{S}_{\mathscr{E}}(G)$ thus have upper bounds, and Zorn's lemma implies $\mathcal{S}_{\mathscr{E}}(G)$ has maximal elements.

Proposition 7.5. $\mathcal{S}_{\mathscr{E}}(G)$ has a unique $\subseteq$-maximal element.

Proof. Suppose $M, N$ are two $\subseteq$-maximal elements. Form $\overline{M N}$ and consider $\overline{M N} / M \cap N$. It is easy to see $\overline{M N} / M \cap N$ is a quasi-product of $M / M \cap N$ and $N / M \cap N$, and these groups are elementary via Theorem 3.18. Two further applications of Theorem 3.18 imply $\overline{M N} / M \cap N \in$ $\mathscr{E}$ and $\overline{M N} \in \mathscr{E}$. We conclude that $M=\overline{M N}=N$, so $S_{\mathscr{E}}(G)$ has a unique maximal element.

We denote the unique maximal element of $\mathcal{S}_{\mathscr{E}}(G)$ by $\operatorname{Rad}_{\mathscr{E}}(G)$. We call $\operatorname{Rad}_{\mathscr{E}}(G)$ the elementary radical of $G$. The elementary radical enjoys two useful properties:

\section{OBSERVATION 7.6.}

(1) $\operatorname{Rad}_{\mathscr{E}}(G)$ is a closed characteristic subgroup of $G$ and contains every element of $\mathcal{S}_{\mathscr{E}}(G)$.

(2) $\operatorname{Rad}_{\mathscr{E}}\left(G / \operatorname{Rad}_{\mathscr{E}}(G)\right)=\{1\}$.

We now explore a notion dual to the elementary radical. Let $G$ be a t.d.l.c.s.c. group and put

$$
\mathcal{Q}_{\mathscr{E}}(G):=\{N \unlhd G \mid G / N \text { is elementary }\} .
$$

We claim $\subseteq$-decreasing chains in $\mathcal{Q}_{\mathscr{E}}(G)$ have lower bounds. Indeed, suppose $\left(N_{\alpha}\right)_{\alpha \in I}$ is an $\subseteq$-decreasing chain in $\mathcal{Q}_{\mathscr{E}}(G)$ and put $N:=\bigcap_{\alpha \in I} N_{\alpha}$. The group $G / N$ is thus residually elementary. Via the permanence properties of $\mathscr{E}, G / N$ is elementary, hence $N \in \mathcal{Q}_{\mathscr{E}}(G)$. We 
conclude that $\subseteq$-decreasing chains in $\mathcal{Q}_{\mathscr{E}}(G)$ have lower bounds, and applying Zorn's lemma, $\mathcal{Q}_{\mathscr{E}}(G)$ has minimal elements.

Proposition 7.7. $\mathcal{Q}_{\mathscr{E}}(G)$ has a unique $\subseteq$-minimal element.

Proof. Suppose $M, N$ are $\subseteq$-minimal elements of $\mathcal{Q}_{\mathscr{E}}(G)$. The usual projection map $M / M \cap$ $N \rightarrow G / N$ is a continuous, injective group homomorphism. Theorem 3.18 thus implies $M / M \cap$ $N$ is elementary, and since $(G / M \cap N) /(M / M \cap N) \simeq G / M$ and $G / M$ is elementary, a second application of Theorem 3.18 implies $G / M \cap N$ is elementary. The minimality of $M$ and $N$ now gives that $M=M \cap N=N$ as required.

We call the unique minimal element of $\mathcal{Q}_{\mathscr{E}}(G)$ the elementary residual of $G$, denoted $\operatorname{Res}_{\mathscr{E}}(G)$. We note two important features of the elementary residual.

\section{OBSERVATION 7.8 .}

(1) $\operatorname{Res}_{\mathscr{E}}(G)$ is a closed characteristic subgroup of $G$ and is contained in every element of $\mathcal{Q}_{\mathscr{E}}(G)$.

(2) $\operatorname{Res}_{\mathscr{E}}\left(\operatorname{Res}_{\mathscr{E}}(G)\right)=\operatorname{Res}_{\mathscr{E}}(G)$.

Collecting our results, we have proved the following theorem:

TheOREm 7.9. Let $G$ be a t.d.l.c.s.c. group. Then

(1) There is a unique maximal closed normal subgroup $\operatorname{Rad}_{\mathscr{E}}(G)$ such that $\operatorname{Rad}_{\mathscr{E}}(G)$ is elementary.

(2) There is a unique minimal closed normal subgroup $\operatorname{Res}_{\mathscr{E}}(G)$ such that $G / \operatorname{Res}_{\mathscr{E}}(G)$ is elementary.

\subsection{The structure of compactly generated groups}

A key ingredient in the proof of the following theorem is the relationship between normal subgroups and $G$-congruences. The inspiration for this approach comes from a fascinating paper of Trofimov [27.

TheOREM 7.10. Let $G$ be a compactly generated t.d.l.c.s.c. group. Then there is a finite series of closed characteristic subgroups

$$
\{1\}=H_{0} \leqslant H_{1} \leqslant \ldots \leqslant H_{n} \leqslant G
$$

such that

(1) $G / H_{n} \in \mathscr{E}$

(2) for $0 \leqslant k \leqslant n-1,\left(H_{k+1} / H_{k}\right) / \operatorname{Rad}_{\mathscr{E}}\left(H_{k+1} / H_{k}\right)$ is a quasi-product with $0<n_{k+1}<\infty$ many topologically characteristically simple non-elementary quasi-factors; and

(3) $n \leqslant \operatorname{deg}(G)$.

Proof. Fix $\Gamma$ a Cayley-Abels graph for $G$ so that $\operatorname{deg}(G)=\operatorname{deg}(\Gamma)$. We inductively build a sequence of characteristic subgroups $\{1\}=H_{0} \leqslant H_{1} \leqslant \ldots \leqslant H_{i}$ so that condition (2) of the theorem holds of successive terms and $\operatorname{deg}\left(\Gamma / \sigma_{j-1}\right)>\operatorname{deg}\left(\Gamma / \sigma_{j}\right)$ for $0<j \leqslant i$ where $\sigma_{j}$ is the $G$-congruence induced by the orbits of $H_{j}$ on $V \Gamma$. 
The base case is immediate with $H_{0}:=\{1\}$. Suppose we have defined $H_{k}$ and pass to $\tilde{G}:=$ $G / H_{k}$. If $\tilde{G} \in \mathscr{E}$, we stop. Else, let $\pi: G \rightarrow \tilde{G} / \operatorname{Rad}_{\mathscr{E}}(\tilde{G})$ be the usual projection. The group $\tilde{G} / \operatorname{Rad}_{\mathscr{E}}(\tilde{G})$ has trivial elementary radical, so $\tilde{G} / \operatorname{Rad}_{\mathscr{E}}(\tilde{G})$ has no non-trivial discrete normal subgroups and trivial locally elliptic radical. Theorem 7.3 thus implies $\tilde{G} / \operatorname{Rad}_{\mathscr{E}}(\tilde{G})$ has exactly $0<n_{k+1}<\infty$ many minimal non-trivial normal subgroups $N_{1}, \ldots, N_{n_{k+1}}$. These subgroups must be non-elementary and topologically characteristically simple.

We claim $\overline{\left\langle N_{1}, \ldots, N_{n_{k+1}}\right\rangle}$ is a quasi-product. Let $m: N_{1} \times \cdots \times N_{n_{k+1}} \rightarrow \overline{\left\langle N_{1}, \ldots, N_{n_{k+1}}\right\rangle}$ be the multiplication map. The minimality of the $N_{i}$ gives that the $N_{i}$ pairwise centralize each other, and thereby, the multiplication map is also a homomorphism. It follows immediately that the image of $m$ is dense. To check that $m$ is injective, take $\left(x_{1}, \ldots, x_{n_{k+1}}\right) \in \operatorname{ker}(m)$ and suppose some $x_{i}$ is non-trivial. Without loss of generality, we may assume $i=1$. We now see that

$$
1 \neq x_{1} \in N_{1} \cap \overline{\left\langle N_{2}, \ldots, N_{n_{k+1}}\right\rangle},
$$

and by the minimality of $N_{1}, N_{1} \leqslant \overline{\left\langle N_{2}, \ldots, N_{n_{k+1}}\right\rangle}$. The group $N_{1}$ commutes with the generators of $\overline{\left\langle N_{2}, \ldots, N_{n_{k+1}}\right\rangle}$, so $N_{1}$ is central in $\overline{\left\langle N_{2}, \ldots, N_{n_{k+1}}\right\rangle}$. Abelian groups, however, are elementary making $N_{1}$ a non-trivial elementary normal subgroup. This contradicts that $\tilde{G} / \operatorname{Rad}_{\mathscr{E}}(\tilde{G})$ has trivial elementary radical. The map $m$ therefore has trivial kernel and is injective. We conclude that $\overline{\left\langle N_{1}, \ldots, N_{n_{k+1}}\right\rangle}$ is a quasi-product.

Putting

$$
H_{k+1}:=\pi^{-1}\left(\overline{\left\langle N_{1}, \ldots, N_{n_{k+1}}\right\rangle}\right)
$$

we have that the successive terms of $H_{0}, \ldots, H_{k}, H_{k+1}$ satisfy (2) of the theorem. It remains to show $\operatorname{deg}\left(\Gamma / \sigma_{k}\right)>\operatorname{deg}\left(\Gamma / \sigma_{k+1}\right)$. Put $R:=\pi^{-1}\left(\operatorname{Rad}_{\mathscr{E}}\left(G / H_{k}\right)\right)$ and let $\tau$ be the $G$-congruence on $V \Gamma$ corresponding to $R$. Since $H_{k} \leqslant R \leqslant H_{k+1}$, we have that

$$
\operatorname{deg}\left(\Gamma / \sigma_{k}\right) \geqslant \operatorname{deg}(\Gamma / \tau) \geqslant \operatorname{deg}\left(\Gamma / \sigma_{k+1}\right),
$$

so it is enough to $\operatorname{show} \operatorname{deg}(\Gamma / \tau)>\operatorname{deg}\left(\Gamma / \sigma_{k+1}\right)$.

Let $\pi: G \rightarrow G / R$ be the usual projection, put $\tilde{H}_{k+1}:=\pi\left(H_{k+1}\right)$, and fix $v^{\tau} \in V \Gamma / \tau$. The group $G / R$ has no non-trivial elementary normal subgroups, so $\tilde{H}_{k+1}$ is non-discrete. We may therefore find $\tilde{h} \in\left(\tilde{H}_{k+1}\right)_{\left(v^{\tau}\right)} \backslash\{1\}$ as $(G / R)_{\left(v^{\tau}\right)}$ is open where $(G / R)_{\left(v^{\tau}\right)}$ is the stabilizer of $v^{\tau}$ in $G / R$. The group $G / R$ also has no non-trivial compact normal subgroup, hence Observation 7.2 implies $G / R \curvearrowright \Gamma / \tau$ faithfully. There is thus $w^{\tau}$ nearest to $v^{\tau}$ such that $\tilde{h} . w^{\tau} \neq w^{\tau}$. It follows there is a neighbour $u^{\tau}$ of $w^{\tau}$ such that $\tilde{h} . u^{\tau}=u^{\tau}$. Fixing $h \in H_{k+1}$ with $\pi(h)=\tilde{h}$, in the quotient graph $\Gamma / \tau$ the vertex $u^{\tau}$ has $\tilde{h} . w^{\tau}=(h . w)^{\tau}$ and $w^{\tau}$ as distinct neighbors. In the quotient graph $\Gamma / \sigma_{k+1}$, however, $(h . w)^{\sigma_{k+1}}=w^{\sigma_{k+1}}$, so $u^{\sigma_{k+1}}$ has at least one less neighbour. Hence, $\operatorname{deg}(\Gamma / \tau)>\operatorname{deg}\left(\Gamma / \sigma_{k+1}\right)$, and we have verified the inductive claim.

Our inductive construction procedure must halt at $n \leqslant \operatorname{deg}(G)$. The resulting series $\{1\}=$ $H_{0} \leqslant H_{1} \leqslant \ldots \leqslant H_{n} \leqslant G$ satisfies the theorem.

Corollary 7.11. Let $G$ be a compactly generated t.d.l.c. group. Then there is a finite series of closed normal subgroups

$$
\{1\} \leqslant H_{0} \leqslant H_{1} \leqslant \ldots \leqslant H_{n} \leqslant G
$$

such that

(1) $H_{0}$ is compact and $G / H_{0}$ is second countable;

(2) $G / H_{n} \in \mathscr{E}$;

(3) for $0 \leqslant k \leqslant n-1,\left(H_{k+1} / H_{k}\right) / \operatorname{Rad}_{\mathscr{E}}\left(H_{k+1} / H_{k}\right)$ is a quasi-product with $0<n_{k+1}<\infty$ many topologically characteristically simple non-elementary quasi-factors; and

(4) $n \leqslant \operatorname{deg}(G)$. 
Proof. Via [16. (8.7)], there is $H_{0} \unlhd G$ such that $H_{0}$ is compact and $G / H_{0}$ is second countable. We now apply Theorem 7.10 to $G / H_{0}$ to find $\tilde{H}_{1} \leqslant \ldots \leqslant \tilde{H}_{n} \leqslant G / H_{0}$ with $n \leqslant \operatorname{deg}\left(G / H_{0}\right) \leqslant \operatorname{deg}(G)$. Letting $\pi: G \rightarrow G / H_{0}$ be the usual projection and putting $H_{i}:=$ $\pi^{-1}\left(\tilde{H}_{i}\right)$ for $1 \leqslant i \leqslant n$, the series $H_{0} \leqslant H_{1} \leqslant \ldots \leqslant H_{n}$ satisfies the theorem.

REMARK 7.12. The length of the characteristic series given by Theorem 7.10 can be arbitrarily long. Additionally, the quasi-factors need not be topologically simple or compactly generated. Example 7.19 demonstrates these phenomena. In the case of l.c.s.c. p-adic Lie groups, however, it is always the case that $n \leqslant 1$ and that the quasi-factors are compactly generated and topologically simple; see $\mathbf{2 9}$.

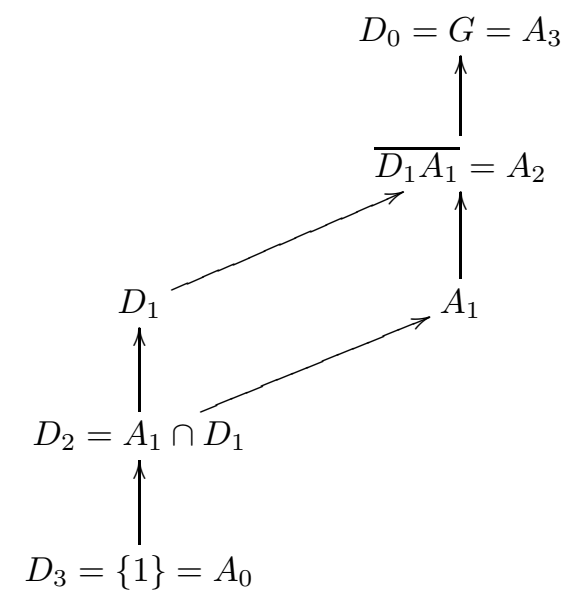

FiguRE 1. The inclusions between the descending and ascending elementary series

\subsection{The descending and ascending elementary series}

In the non-compactly generated case, we obtain weaker, nonetheless useful structure theorems. Via the elementary radical and elementary residual, we build two characteristic subgroup series in a t.d.l.c.s.c. group. Figure 1 indicates the relationship between these series; the arrows indicate inclusion. The diagram follows from the permanence properties of $\mathscr{E}$.

DeFINITION 7.13. For a t.d.l.c.s.c. group $G$ the descending elementary series is defined by $D_{0}:=G, D_{1}:=\operatorname{Res}_{\mathscr{E}}\left(D_{0}\right), D_{2}:=\operatorname{Rad}_{\mathscr{E}}\left(D_{1}\right)$, and $D_{3}:=\{1\}$.

Definition 7.14. For a t.d.l.c.s.c. group $G$ the ascending elementary series is defined by $A_{0}:=\{1\}, A_{1}:=\operatorname{Rad}_{\mathscr{E}}(G), A_{2}:=\pi^{-1}\left(\operatorname{Res}_{\mathscr{E}}\left(G / A_{1}\right)\right)$ where $\pi: G \rightarrow G / A_{1}$ is the usual projection, and $A_{3}:=G$.

DEFINITIOn 7.15. We say a t.d.l.c.s.c. group is elementary-free if it has no non-trivial elementary normal subgroups and no non-trivial elementary quotients. 
Theorem 7.16. Let $G$ be a t.d.l.c.s.c. group. Then the descending elementary series

$$
G \geqslant D_{1} \geqslant D_{2} \geqslant\{1\}
$$

is a series of closed characteristic subgroups with $G / D_{1}$ elementary, $D_{1} / D_{2}$ elementary-free, and $D_{2}$ elementary.

Proof. It is immediate that $D_{1}, D_{2}$ are closed and characteristic and that $G / D_{1}$ and $D_{2}$ are elementary. For the remaining claim, it suffices to show $\operatorname{Rad}_{\mathscr{E}}\left(D_{1} / D_{2}\right)=\{1\}$ and $\operatorname{Res}_{\mathscr{E}}\left(D_{1} / D_{2}\right)=D_{1} / D_{2}$. The former is immediate since $D_{2}=\operatorname{Rad}_{\mathscr{E}}\left(D_{1}\right)$.

For the case of $\operatorname{Res}_{\mathscr{E}}\left(D_{1} / D_{2}\right)$, let $\pi: D_{1} \rightarrow D_{1} / D_{2}$ be the usual projection and put $H:=$ $\pi^{-1}\left(\operatorname{Res}_{\mathscr{E}}\left(D_{1} / D_{2}\right)\right)$. We see $H$ is the intersection of all normal subgroups of $D_{1}$ extending $D_{2}$ whose quotient is elementary. Since $D_{2}$ is characteristic in $D_{1}, H$ is characteristic in $D_{1}$ and, thus, is normal in $G$. We now have a short exact sequence of topological groups

$$
1 \rightarrow D_{1} / H \rightarrow G / H \rightarrow G / D_{1} \rightarrow 1 .
$$

This sequence shows that $G / H$ is a group extension of elementary groups and, therefore, elementary. Since $D_{1}$ is minimal in the collection of normal subgroups whose quotients are elementary, we conclude that $D_{1}=H$. Hence, $\operatorname{Res}_{\mathscr{E}}\left(D_{1} / D_{2}\right)=D_{1} / D_{2}$ as required.

TheOREm 7.17. Let $G$ be a t.d.l.c.s.c. group. Then the ascending elementary series

$$
\{1\} \leqslant A_{1} \leqslant A_{2} \leqslant G
$$

is a series of closed characteristic subgroups with $A_{1}$ elementary, $A_{2} / A_{1}$ elementary-free, and $G / A_{2}$ elementary.

Proof. It is immediate that $A_{1}, A_{2}$ are closed and characteristic and that $A_{1}$ and $G / A_{2}$ are elementary. For the remaining claim, it again suffices to show $\operatorname{Rad}_{\mathscr{E}}\left(A_{2} / A_{1}\right)=\{1\}$ and $\operatorname{Res}_{\mathscr{E}}\left(A_{2} / A_{1}\right)=A_{2} / A_{1}$. For the former, $\operatorname{Rad}_{\mathscr{E}}\left(A_{2} / A_{1}\right)$ is characteristic in $A_{2} / A_{1}$ and, therefore, normal in $G / A_{1}$. Letting $\pi: G \rightarrow G / A_{1}$ be the usual projection, we see $\pi^{-1}\left(\operatorname{Rad}_{\mathscr{E}}\left(A_{2} / A_{1}\right)\right)$ is a elementary normal subgroup of $G$. By choice of $A_{1}$, it must be the case $\pi^{-1}\left(\operatorname{Rad}_{\mathscr{E}}\left(A_{2} / A_{1}\right)\right)=$ $A_{1}$, so $\operatorname{Rad}_{\mathscr{E}}\left(A_{2} / A_{1}\right)=\{1\}$.

For $\operatorname{Res}_{\mathscr{E}}\left(A_{2} / A_{1}\right)$, put $H:=\operatorname{Res}_{\mathscr{E}}\left(A_{2} / A_{1}\right)$. Since $H$ is characteristic in $A_{2} / A_{1}$, we have that $H$ is normal in $G / A_{1}$. This gives a short exact sequence of topological groups:

$$
1 \rightarrow\left(A_{2} / A_{1}\right) / H \rightarrow\left(G / A_{1}\right) / H \rightarrow\left(G / A_{1}\right) /\left(A_{2} / A_{1}\right) \rightarrow 1 .
$$

This sequence shows that $\left(G / A_{1}\right) / H$ is a group extension of elementary groups and, therefore, elementary. Since $A_{2} / A_{1}$ is minimal with respect to normal subgroups of $G / A_{1}$ whose quotient is elementary, $A_{2} / A_{1}=H$ as required.

Theorem 1.10 now follows from either Theorem 7.16 or Theorem 7.17 We note these theorems give different decompositions. It is also worth noting $D_{1} / D_{2}$ and $A_{2} / A_{1}$ need not be compactly generated when $G$ is compactly generated. Examples 7.19 and 7.20 demonstrate these phenomena.

Question 7.18 (Caprace). Are $D_{1} / D_{2}$ and $A_{2} / A_{1}$ isomorphic? Is the obvious inclusion $D_{1} / D_{2} \rightarrow A_{2} / A_{1}$ necessarily an isomorphism of topological groups? 


\subsection{Examples}

Fix a prime $p$ and $U \in \mathcal{U}\left(P S L_{3}\left(\mathbb{Q}_{p}\right)\right)$ and let $\left(G_{i}\right)_{i \in \mathbb{Z}}$ list countably many copies of $P S L_{3}\left(\mathbb{Q}_{p}\right)$ with $U_{i}$ the copy of $U$ in $G_{i}$. Putting $G:=\bigoplus_{i \in \mathbb{Z}}\left(G_{i}, U_{i}\right)$, one verifies $G$ has non-trivial proper normal subgroups, is elementary free, and is not compactly generated. It will be convenient to see the group $G$ as the set of functions $f: \mathbb{Z} \rightarrow P S L_{3}\left(\mathbb{Q}_{p}\right)$ so that $f(i) \in U$ for all but finitely $i$; cf. the discussion after Definition 2.3.

Example 7.19. We use $G$ to show the length of the characteristic series in Theorem 7.10 can be arbitrarily long. These examples are due to Pierre-Emmanuel Caprace; the author thanks Caprace for allowing these to be included in the present work work.

There is a natural action of $\mathbb{Z}$ on $G$ by shifting the coordinates; that is to say, $\mathbb{Z} \curvearrowright G$ by $(n . f)(i):=f(i-n)$. We may thus form the t.d.l.c.s.c. group $L_{1}:=G \rtimes \mathbb{Z}$ where $\mathbb{Z}$ acts on $G$ by shift. Let $X$ be a compact generating set for $P S L_{3}\left(\mathbb{Q}_{p}\right)$ and put $K:=\prod_{i<0} U_{i} \times X \times \prod_{i>0} U_{i}$. Taking $(1, t)$ a generator for $\mathbb{Z}$ in $L_{1}$, it follows that $K \cup\{(1, t)\}$ is a compact generating set for $L_{1}$. We conclude that $L_{1}$ is a compactly generated t.d.l.c.s.c. group, so we may compute the decomposition given by Theorem 7.10 . One verifies that $\operatorname{Rad}_{\mathscr{E}}\left(L_{1}\right)$ is trivial and that $G$ is the unique minimal non-trivial normal subgroup of $L_{1}$. It then follows that the decomposition is

$$
\{1\} \leqslant G \leqslant L_{1} .
$$

We now show how to produce a group with a longer decomposition. Fix $U \in \mathcal{U}\left(L_{1}\right)$. Since $L_{1}$ has no non-trivial compact normal subgroups, $L_{1}$ acts on the set of left cosets of $U$ continuously, transitively, and faithfully. By enumerating the collection of cosets by $\mathbb{Z}, L_{1} \curvearrowright \mathbb{Z}$ continuously, transitively, and faithfully by permutations. As in the discussion after Definition 2.3 , there is a group action $L_{1} \curvearrowright G$ given by permuting the coordinates. We now consider the t.d.l.c.s.c. group $G \rtimes L_{1}=: L_{2}$. Similar to the previous example, $L_{2}$ is compactly generated with trivial elementary radical and with $G$ as the unique minimal non-trivial normal subgroup. The decomposition given by Theorem 7.10 is thus

$$
\{1\} \leqslant G \leqslant G \rtimes G \leqslant L_{2} .
$$

Continuing in this fashion, we produce compactly generated t.d.l.c.s.c. groups such that the series given by Theorem 7.10 is arbitrarily long.

We remark that $L_{1}$ shows we may not assume the topologically characteristically simple quasi-factors are topologically simple or compactly generated. One verifies that $L_{1}$ additionally shows $D_{1} / D_{2}$ and $A_{2} / A_{1}$ need not be compactly generated for a compactly generated group.

EXAmple 7.20. Again using $G$, we produce a t.d.l.c.s.c. group such that all inclusions of the descending elementary series are proper. Let $V:=\prod_{i \in \mathbb{Z}} U_{i} \in \mathcal{U}(G)$ and let $\mathcal{D}$ be the countable collection of left cosets of $V$ in $G$. Certainly, $G \curvearrowright \mathcal{D}$ continuously and transitively by left multiplication; this action is also faithful since $G$ has no non-trivial compact normal subgroups. As in the previous example, $G \curvearrowright A_{5}^{\mathcal{D}}$ by shift, so we may form $H:=\left(A_{5}^{\mathcal{D}} \rtimes G\right) \times \mathbb{Z}$. The descending elementary series for $H$ is

$$
H \geqslant G \ltimes A_{5}^{\mathcal{D}} \geqslant A_{5}^{\mathcal{D}} \geqslant\{1\},
$$

and all of the inclusions in this series are proper.

We remark that $H$ also demonstrates that the descending and ascending elementary series may differ. The ascending elementary series of $H$ has $A_{1}=A_{5}^{\mathcal{D}} \times \mathbb{Z}$ and $A_{2}=H$. 


\section{Application 2: Locally solvable groups}

For our second application, we prove local-to-global results for the class of t.d.l.c.s.c. group with an open solvable subgroup. Such groups are called locally solvable. In the case the open solvable subgroup is nilpotent, we call these groups locally nilpotent.

\subsection{Preliminaries}

The derived series of a topological group $G$ is the sequence of closed normal subgroups $\left(G^{(k)}\right)_{k \geqslant 0}$ defined by $G^{(0)}:=G$ and $G^{(n+1)}:=\overline{\left[G^{(n)}, G^{(n)}\right]}$. We say $G$ is solvable if the derived series stabilizes at $\{1\}$ after finitely many steps. For solvable $G$, the derived length, $l(G)$, is the least $k$ such that $G^{(k)}=\{1\}$.

The lower central series of a topological group $G$ is the sequence of closed normal subgroups $\left(G_{k}\right)_{k \geqslant 1}$ defined by $G_{1}:=G$ and $G_{n+1}:=\overline{\left[G, G_{n}\right]}$. We say $G$ is nilpotent if the lower central series stabilizes at $\{1\}$ after finitely many steps. The nilpotence class of nilpotent $G, n(G)$, is the least $k$ so that $G_{k}=\{1\}$. Observe that the last non-trivial term in the lower central series of a nilpotent topological group is central.

\subsection{The structure of locally solvable groups}

For a locally solvable t.d.l.c.s.c. group $G$, define

$$
r_{s}(G):=\min \{l(U) \mid U \in \mathcal{U}(G) \text { and is solvable }\} .
$$

We call $r_{s}(G)$ the solvable rank of $G$. A t.d.l.c.s.c. group is locally solvable of solvable rank zero if and only if it is a countable discrete group.

THEOREm 8.1. If $G$ is a locally solvable t.d.l.c.s.c. group, then $G \in \mathscr{E}$ with $\operatorname{rk}(G) \leqslant 4^{r_{s}(G)}$.

Proof. We induct on $r_{s}(G)$ for the theorem. The base case case, $r_{s}(G)=0$, is immediate since such a group is discrete.

Suppose $G$ is a locally solvable group with $r_{s}(G)=k+1$. Let $U \in \mathcal{U}(G)$ be solvable with derived length $k+1$ and put

$$
\mathcal{A}_{k+1}:=\left\{C \in S(G) \mid \forall V \in \mathcal{U}(G) \exists \text { solvable } W \leqslant \leqslant_{o} V: C \leqslant N_{G}\left(W^{(k)}\right)\right\} .
$$

Certainly, $\mathcal{A}_{k+1}$ is hereditary. To see conjugation invariance, take $C \in \mathcal{A}_{k+1}, V \in \mathcal{U}(G)$, and $g \in G$. Find $W \leqslant{ }_{o} g^{-1} V g$ such that $C \leqslant N_{G}\left(W^{(k)}\right)$. We thus have that

$$
g C g^{-1} \leqslant N_{G}\left(g W^{(k)} g^{-1}\right)=N_{G}\left(\left(g W g^{-1}\right)^{(k)}\right)
$$

and $g W g^{-1} \leqslant_{o} V$. It now follows that $g C g^{-1} \in \mathcal{A}_{k+1}$, whereby $\mathcal{A}_{k+1}$ is invariant under conjugation.

By way of Proposition 2.10, we may form the $\mathcal{A}_{k+1}$-core; denote this subgroup by $N_{k+1}$.

Claim. $U^{(k)} \leqslant N_{k+1}$.

Proof of Claim. Take $u \in U^{(k)}, C \in \mathcal{A}_{k+1}$, and $V \in \mathcal{U}(G)$. It suffices to show there is $W \leqslant_{o} V$ such that $\overline{\langle u, C\rangle} \leqslant N_{G}\left(W^{(k)}\right)$. By definition of $\mathcal{A}_{k+1}$, there is $W \leqslant_{o} U \cap V$ with $C \leqslant N_{G}\left(W^{(k)}\right)$. Since $W^{(k)} \leqslant U^{(k)}$ and $U^{(k)}$ is abelian, $u \in N_{G}\left(W^{(k)}\right)$. Therefore, $\overline{\langle u, C\rangle} \leqslant$ $N_{G}\left(W^{(k)}\right)$ as required.

Put $N=\overline{N_{k+1}}$. By the claim, $r_{s}(G / N) \leqslant k$, and the induction hypothesis implies $G / N \in \mathscr{E}$ with $\operatorname{rk}(G / N) \leqslant 4^{k}$. On the other hand, let $\left(n_{i}\right)_{i \in \omega}$ list a countable dense subset of $N_{k+1}$. 
Our group $N$ is an increasing union of $M_{i}:=\left\langle U \cap N, n_{0}, \ldots, n_{i}\right\rangle$. Moreover, by the choice of $n_{0}, \ldots, n_{i}$, the group $M_{i}$ is a member of $\mathcal{A}_{k+1}$ for each $i$ since $U \in \mathcal{A}_{k+1}$. We may thus find $W \leqslant{ }_{o} U$ such that $M_{i}$ normalizes $W^{(k)}$. So $W^{(k)} \cap M_{i} \leqslant \operatorname{Rad}_{\mathcal{L E}}\left(M_{i}\right)$, and since $\left(W \cap M_{i}\right)^{(k)} \leqslant$ $W^{(k)} \cap M_{i}$, we infer that $r_{s}\left(M_{i} / \operatorname{Rad}_{\mathcal{L E}}\left(M_{i}\right)\right) \leqslant k$. The induction hypothesis now implies $M_{i} / \operatorname{Rad}_{\mathcal{L E}}\left(M_{i}\right) \in \mathscr{E}$ with $\operatorname{rk}\left(M_{i} / \operatorname{Rad}_{\mathcal{L E}}\left(M_{i}\right)\right) \leqslant 4^{k}$. The $\operatorname{group} \operatorname{Rad}_{\mathcal{L E}}\left(M_{i}\right)$ is elementary with rank at most 1 , whereby Proposition 3.5 gives that $M_{i} \in \mathscr{E}$ with $\operatorname{rk}\left(M_{i}\right) \leqslant 4^{k}+2$. It now follows that $N \in \mathscr{E}$ with $\operatorname{rk}(N) \leqslant 4^{k}+3$.

A second application of Proposition 3.5 implies $G \in \mathscr{E}$ with

$$
\operatorname{rk}(G) \leqslant 4^{k}+4^{k}+4 \leqslant 4^{k+1} .
$$

This completes the induction, and we conclude the theorem.

As a corollary, we recover a theorem of Willis.

Corollary 8.2 (Willis, 30, Theorem 2.2]). If $G$ is a non-discrete, compactly generated, t.d.l.c. group that is topologically simple, then $G$ is not locally solvable.

Proof. Suppose for contradiction $G$ is locally solvable. By [16. (8.7)], $G$ is second countable, and we conclude that $G \in \mathscr{E}$ via Theorem 8.1. Proposition 6.3 then implies $G$ is discrete which contradicts our assumptions on $G$.

The proof of Theorem 8.1 gives a procedure for decomposing a locally solvable t.d.l.c.s.c. group into groups that are either profinite, discrete, or have smaller solvable rank. The proof also implies groups with a compact open abelian subgroup have a nice structure. We record these observations here:

Proposition 8.3. If $G$ is a locally abelian t.d.l.c.s.c. group, then $\operatorname{SIN}(G) \unlhd_{o} G$.

TheOREm 8.4. Suppose a t.d.l.c.s.c. group $G$ is locally solvable and $r_{s}(G) \geqslant 1$. Then there is $1 \leqslant k \leqslant r_{s}(G)$ and a sequence of closed characteristic subgroups

$$
\{1\}=: N_{0} \leqslant N_{1} \leqslant \ldots \leqslant N_{k} \leqslant G
$$

such that

(1) $r_{s}(G)>r_{s}\left(G / N_{1}\right)>\cdots>r_{s}\left(G / N_{k}\right)=0$, and

(2) for all $1 \leqslant j \leqslant k, N_{j} / N_{j-1}=\bigcup_{i \in \omega} H_{i}$ with $\left(H_{i}\right)_{i \in \omega}$ an $\subseteq$-increasing sequence of compactly generated open subgroups of $N_{j} / N_{j-1}$ for which $r_{s}\left(H_{i} / \operatorname{Rad}_{\mathcal{L E}}\left(H_{i}\right)\right)<r_{s}\left(G / N_{j-1}\right)$ for all $i \in \omega$.

\subsection{The structure of locally nilpotent groups}

The nilpotence rank of a locally nilpotent t.d.l.c.s.c. group $G$ is defined to be

$$
r_{n}(G):=\min \{n(U) \mid U \in \mathcal{U}(G) \text { and is nilpotent }\}
$$

where $n(U)$ is the nilpotence class of $U$. A t.d.l.c.s.c. group has nilpotence rank 1 if and only if it is discrete. 
TheOREM 8.5. Suppose $G$ is a locally nilpotent t.d.l.c.s.c. group. Then there is $1 \leqslant k \leqslant$ $r_{n}(G)$ and a sequence of closed characteristic subgroups

$$
\{1\}=: N_{0} \leqslant N_{1} \leqslant \ldots \leqslant N_{k} \leqslant{ }_{o} G
$$

such that $N_{i} / N_{i-1} \simeq \operatorname{SIN}\left(G / N_{i-1}\right)$ for $1 \leqslant i \leqslant k$.

Proof. We proceed by induction on $i$ to build the $N_{i}$. For the base case, put $N_{1}:=\operatorname{SIN}(G)$; recall $\operatorname{SIN}(G)$ is a closed characteristic subgroup. If $N_{1}=G$, then we stop. Else, suppose $N_{1} \neq G$ and let $U \in \mathcal{U}(G)$ be such that $n(U)=r_{n}(G)$. The group $G$ must be non-discrete, so $n(U / Z(U))<n(U)$. Since $Z(U) \leqslant \operatorname{SIN}(G)$, we conclude that $r_{n}\left(G / N_{1}\right)<r_{n}(G)$.

Suppose we have defined a closed characteristic $N_{l}$ for some $l \geqslant 1$. If $r_{n}\left(G / N_{l}\right)=1$, we stop. Else, let $\pi_{l}: G \rightarrow G / N_{l}$ be the usual projection and put $N_{l+1}:=\pi_{l}^{-1}\left(\operatorname{SIN}\left(G / N_{l}\right)\right)$. We now have that $N_{l+1}$ is closed and characteristic, $N_{l+1} / N_{l} \simeq \operatorname{SIN}\left(G / N_{l}\right)$, and $G / N_{l+1} \simeq$ $\left(G / N_{l}\right) / \operatorname{SIN}\left(G / N_{l}\right)$. Letting $U \in \mathcal{U}\left(G / N_{l}\right)$ be such that $n(U)=r_{n}\left(G / N_{l}\right)$, we additionally see that the group $\operatorname{SIN}\left(G / N_{l}\right)$ contains $Z(U)$, so $r_{n}\left(G / N_{l+1}\right)<r_{n}\left(G / N_{l}\right)$.

Since the nilpotence rank of $G / N_{l}$ drops at each stage of our construction, our procedure halts at some $k \leqslant n(U)$. At this $k$, it must be the case that $r_{n}\left(G / N_{k}\right)=1$, and therefore, $G / N_{k}$ is discrete. We conclude that

$$
N_{1} \leqslant N_{2} \leqslant \ldots \leqslant N_{k} \leqslant o G
$$

satisfies the theorem.

REMARK 8.6. The results in this section demonstrate that local assumptions affect global structure even in the setting of elementary groups.

\subsection{Examples}

We here present examples illustrating Proposition 8.3 and Theorem 8.4 .

EXAmple 8.7. For Proposition 8.3 , let $F:=\mathbb{Z} / 3 \mathbb{Z}$ and $\left(F_{i}\right)_{i \in \mathbb{Z}}$ list countably many copies of $F$. Set $U_{i}=F$ for $i \leqslant 0$ and $U_{i}=\{1\}$ for $i>0$ and form $\bigoplus_{i \in \mathbb{Z}}\left(F_{i}, U_{i}\right)$. Since $\mathbb{Z} \curvearrowright \bigoplus_{i \in \mathbb{Z}}\left(F_{i}, U_{i}\right)$ by shift, we may form

$$
G:=\bigoplus_{i \in \mathbb{Z}}\left(F_{i}, U_{i}\right) \rtimes \mathbb{Z} ;
$$

see the discussion after Definition 2.3 for more details. The group $\prod_{i \leqslant 0} F_{i}$ is a compact open subgroup of $G$, hence $G$ is locally abelian. One verifies that

$$
\bigoplus_{i \in \mathbb{Z}}\left(F_{i}, U_{i}\right)=\operatorname{SIN}(G)
$$

Example 8.8. For Theorem 8.4 , let $P$ be the non-discrete topologically simple locally elliptic group with a compact open abelian subgroup built by Willis [30, Proposition 3.4]. Let $V \in \mathcal{U}(P)$ be abelian and define $\mathcal{D}$ to be the collection of left cosets of $V$ in $P$. The group $P$ acts on $\mathcal{D}$ by left multiplication, and this action is continuous, faithful, and transitive. Take $S$ to be the finitely generated infinite simple group built by Higman $[17$ and set $F:=\mathbb{Z} / 3 \mathbb{Z}$. We now form $S^{<\mathcal{D}}$ and $F^{S^{<\mathcal{D}}}$, and since $S^{<\mathcal{D}}$ acts on $F^{S^{<\mathcal{D}}}$ by shift, we may construct the t.d.l.c.s.c. group $F^{S^{<\mathcal{D}}} \rtimes S^{<\mathcal{D}}$. We may further construct

$$
H:=\left(F^{S^{<\mathcal{D}}} \rtimes S^{<\mathcal{D}}\right) \rtimes P
$$


where $P \curvearrowright\left(F^{S^{<\mathcal{D}}} \rtimes S^{<\mathcal{D}}\right)$ via $p .(\alpha, f):=(p . \alpha, p . f)$ with $p . f$ the usual shift and $p . \alpha$ is the shift induced by the shift action of $P$ on $S^{<\mathcal{D}}$.

The subgroup

$$
U:=\left(F^{S^{<\mathcal{D}}} \times\{1\}\right) \times V
$$

is a compact open two step solvable subgroup of $H$. The group $H$ acts on the set of left cosets of $U$, denoted $\mathcal{F}$, transitively with $F^{S^{<\mathcal{D}}}$ in the kernel of the action. We take a second semi-direct product to obtain

$$
G:=S^{<\mathcal{F}} \rtimes H
$$

where $H \curvearrowright S^{<\mathcal{F}}$ by shift. Since $U$ remains a compact open subgroup of $G, G$ is locally solvable with $r_{s}(G)=2$.

We now compute the decomposition given by Theorem 8.4. One verifies that $N_{1}=S^{<\mathcal{F}} \rtimes$ $F^{S^{<\mathcal{D}}}$. Since $F^{S^{<\mathcal{D}}}$ acts trivially on $S^{<\mathcal{F}}$, this semi-direct product is indeed a direct product. Setting $H_{n}:=S^{n} \times F^{S^{<\mathcal{D}}}$, we have that $N_{1}=\bigcup_{n \geqslant 0} H_{n}$, and for each $n \geqslant 0, H_{n} / \operatorname{Rad}_{\mathcal{L E}}\left(H_{n}\right)$ is discrete and thereby has solvable rank zero.

On the other hand, $G / N_{1}=S^{<\mathcal{D}} \rtimes P$ and thus is locally abelian. Since $P$ is topologically simple, it follows that $N_{2}=\psi^{-1}\left(\operatorname{SIN}\left(G / N_{1}\right)\right)=G$, hence $N_{2} / N_{1}=\bigcup_{n \geqslant 0} O_{n}$ with $\left(O_{n}\right)_{n \geqslant 0}$ an $\subseteq$-increasing sequence of compactly generated $S I N$ groups. The group $O_{n} / \operatorname{Rad}_{\mathcal{L E}}\left(O_{n}\right)$ is discrete and so has solvable rank zero. We have now computed the decomposition for $G$ as given by Theorem 8.4 .

$$
\{1\} \leqslant S^{<\mathcal{F}} \rtimes F^{S^{<\mathcal{D}}} \leqslant N_{2}=G .
$$

\section{Application 3: $[A]$-regular groups}

For our last application, we consider a somewhat technical local assumption, which plays an important role in the new, deep theory developed in [9] and [10. Specifically, we consider the structure of t.d.l.c.s.c. groups that are $[A]$-regular.

\subsection{Preliminaries}

These preliminary definitions and facts either come from or are implicit in $\mathbf{9}$; we include a discussion for completeness.

Definition 9.1. Let $[A]$ be the smallest class of profinite groups such that the following conditions hold:

(i) $[A]$ contains all abelian profinite groups and all finite simple groups.

(ii) If $U \in[A]$ and $K \unlhd U$, then $K \in[A]$ and $U / K \in[A]$.

(iii) If $U=V_{1} \ldots V_{n}$ with $V_{i} \unlhd U$ and $V_{i} \in[A]$ for each $1 \leqslant i \leqslant n$, then $U \in[A]$.

It is an easy application of Fitting's theorem to see $[A]$ consists of virtually nilpotent profinite groups. For a profinite group $U$, we let $[A](U)$ denote the closed subgroup generated by all normal subgroups of $U$ that are members of the class $[A]$.

For $G$ a t.d.l.c. group, a compact $K \leqslant G$ is locally normal if $N_{G}(K)$ is open. Via locally normal subgroups, we arrive at the central definition of this section.

Definition 9.2. Let $U$ be a profinite group and $G$ be a t.d.l.c. group. 
(i) For $K \leqslant U$ a closed subgroup, $K$ is $[A]$-regular if for every $L \unlhd U$ closed that does not contain $K$, the image of $K$ in $U / L$ contains a non-trivial locally normal $[A]$-subgroup of $U / L$.

(ii) For $H \leqslant G$ a closed subgroup, $H$ is $[A]$-regular if $U \cap H$ is $[A]$-regular in $U$ for all $U \in$ $\mathcal{U}(G)$.

(iii) $G$ is $[A]$-semisimple if $G$ has trivial quasi-centre and the only locally normal subgroup of $G$ belonging to $[A]$ is $\{1\}$

In particular, a t.d.l.c.s.c. group $G$ is $[A]$-regular, if for every $U \in \mathcal{U}(G)$ and every non-trivial quotient group $U / L$, it is the case that $U / L$ contains a non-trivial locally normal [A]-subgroup. As a corollary, if $G$ is $[A]$-regular, then $G / N$ is $[A]$-regular for any closed normal subgroup $N$ of $G$.

We now recall a few basic lemmas regarding locally normal $[A]$-subgroups and a deep result of Caprace, Reid, and Willis, which elucidates the connection between $[A]$-regularity and $[A]$ semisimplicity.

Lemma 9.3. If a profinite group $U$ contains an infinite locally normal $[A]$-subgroup, then $U$ contains an infinite normal $[A]$-subgroup.

Proof. Suppose $L \leqslant U$ is an infinite locally normal $[A]$-subgroup and find $W \unlhd_{o} U$ such that $W \leqslant N_{U}(L)$. Since $L \cap W \unlhd L, L \cap W$ is an [A]-subgroup, and since $L$ is infinite and $W$ is open, $L \cap W$ is also infinite.

Take $u_{1}, \ldots, u_{n}$ left coset representatives for $W$ in $U$. Since $L \cap W$ is an $[A]$-subgroup and $u_{i}(L \cap W) u_{i}^{-1} \unlhd W$ for each $i$, we have that

$$
K:=u_{1}(L \cap W) u_{1}^{-1} \ldots u_{n}(L \cap W) u_{n}^{-1}
$$

is an $[A]$-subgroup. Further, $K \unlhd U$ giving an infinite $[A]$-subgroup that is normal in $U$.

Proposition 9.4. If $G$ is a t.d.l.c. group, then $[A](U) \sim_{c}[A](V)$ for all $U, V \in \mathcal{U}(G)$.

Proof. Fix $U \in \mathcal{U}(G)$. We first show $[A](W) \sim_{c}[A](U)$ for $W \unlhd_{o} U$. Let $A \unlhd W$ be an $[A]$ subgroup of $W$ and fix $u_{1}, \ldots, u_{n}$ left coset representatives for $W$ in $U$. As in the previous proof, $L:=u_{1} A u_{1}^{-1} \ldots u_{n} A u_{n}^{-1}$ is a normal $[A]$-subgroup in $U$, so $A \leqslant L \leqslant[A](U)$. We conclude that $[A](W) \leqslant[A](U)$.

On the other hand, take $a \in[A](U) \cap W$. It suffices to assume $a \in A \unlhd U$ with $A$ some $[A]$ subgroup since $W$ is open. Since $W \cap A \unlhd A$, the group $W \cap A$ is a normal $[A]$-subgroup of $W$, hence $a \in W \cap A \leqslant[A](W)$. We now have that $[A](U) \cap W \leqslant[A](W) \leqslant[A](U)$, and therefore, $[A](U) \sim_{c}[A](W)$.

For an arbitrary $V \in \mathcal{U}(G)$, we may find $W \unlhd_{o} V$ such that $W \unlhd_{o} U \cap V$. By our argument above,

$$
[A](V) \sim_{c}[A](W) \sim_{c}[A](U \cap V) .
$$

On the other hand, the same argument implies $[A](U) \sim_{c}[A](U \cap V)$. Therefore, $[A](U) \sim_{c}$ $[A](V)$ by the transitivity of $\sim_{c}$.

Theorem 9.5 (Caprace, Reid, Willis [9, Theorem 6.10]). If $G$ is a t.d.l.c. group, then $G$ has a closed normal subgroup $R_{[A]}(G)$, the $[A]$-regular radical of $G$, such that

(1) $R_{[A]}(G)$ is the unique largest closed normal subgroup of $G$ that is $[A]$-regular. 
(2) $G / R_{[A]}(G)$ is $[A]$-semisimple, and given any closed normal subgroup $N$ of $G$ such that $G / N$ is $[A]$-semisimple, $R_{[A]}(G) \leqslant N$.

\subsection{Quasi-centralizers and commensurators}

We now prove a series of technical lemmas which are used in the next section to show $\overline{Q C}_{G}(U /[A](U))$ is elementary in a t.d.l.c.s.c. group $G$ with a trivial quasi-centre.

Lemma 9.6. Suppose $U$ is a profinite group, $B \unlhd U$, and $U / B$ has a dense quasi-centre. If $A \unlhd U$ and $A \leqslant_{o} B$, then $U / A$ has a dense quasi-centre.

Proof. Fix $\Sigma:=\left(g_{i}\right)_{i \in I} \subseteq U$ coset representatives for the elements of $Q Z(U / B)$ in $U / B$. Consider the set of elements of $U / A$ with the form $g_{i} b A$ for $i \in I$ and $b \in B$. By the choice of the $g_{i}$, for each $i \in I$ there is $W_{i} \leqslant{ }_{o} U$ such that $\left[g_{i}, W_{i}\right] \subseteq B$. In fact, more is true: for any $b \in B$ and $w \in W_{i}$, we have that $\left[g_{i} b, w\right]=[b, w]^{g_{i}}\left[g_{i}, w\right] \in B$.

Now the map $\xi: W_{i} \rightarrow U / A$ by $w \mapsto\left[g_{i} b, w\right] A$ is continuous and by the above, has image in $B / A$. Since $1 \in i m(\xi)$ and $B / A$ is finite, there is $V_{i} \leqslant_{o} W_{i}$ such that $\xi\left(V_{i}\right)=1$. It follows that $C_{U / A}\left(g_{i} b A\right)$ is open for each $i \in I$ and $b \in B$. Therefore, $\left\{g_{i} b A \mid b \in B\right.$ and $\left.i \in I\right\} \subseteq Q Z(U / A)$.

On the other hand, the map $\psi: U / A \rightarrow U / B$ defined by $u A \mapsto u B$ is open and continuous. It follows the set $\left\{g_{i} b A \mid b \in B\right.$ and $\left.i \in I\right\}$ is additionally dense in $U / A$. Thus, $U / A$ has a dense quasi-centre.

Lemma 9.7. Suppose $G$ is a t.d.l.c.s.c. group. Suppose further there is $U \in \mathcal{U}(G)$ with a virtually nilpotent $B \unlhd U$ such that $\operatorname{Comm}_{G}(B)=G$ and $Q Z(U / B)$ is dense in $U / B$. Then $G$ is elementary.

Proof. Given a triple $(G, U, B)$ as hypothesized, we may find $W \unlhd_{o} U$ such that $A:=W \cap B$ is nilpotent. Plainly, $A \unlhd U, A$ is nilpotent, and $\operatorname{Comm}_{G}(A)=G$. We induct on the minimal nilpotence class of such an $A$ for the lemma. For the base case, $n(A)=1$, the group $A$ is trivial. Lemma 9.6 thus implies $U=U / A$ has a dense quasi-centre. Since $Q Z(G) \leqslant \operatorname{SIN}(G)$, we conclude that $\operatorname{SIN}(G)$ is open in $G$, and it follows that $G \in \mathscr{E}$.

Suppose the lemma holds for all triples $(G, U, B)$ as hypothesized such that there is a nilpotent $A \leqslant_{o} B$ with $A \unlhd U$ and $n(A) \leqslant k$. Suppose the triple $(G, U, B)$ is as hypothesized, but every $A \leqslant_{0} B$ with $A \unlhd U$ and $A$ nilpotent has $n(A)=k+1$. Fix such an $A$.

Define $\mathcal{H} \subseteq S(G)$ by $C \in \mathcal{H}$ if and only if for all $V \in \mathcal{U}(G)$, there exists $N_{W} \unlhd W \leqslant_{o} V$ and $\left(W_{i}\right)_{i \in \omega}$ a normal basis at 1 for $W$ such that

(i) $N_{W} \sim_{c} A$, and

(ii) $C \leqslant \bigcap_{i \in \omega} N_{G}\left(N_{W} \cap W_{i}\right)$.

It is immediate that $\mathcal{H}$ is hereditary and that $U \in \mathcal{H}$.

Claim. $\quad \mathcal{H}$ is conjugation invariant.

Proof of Claim. Take $C \in \mathcal{H}$ and $g \in G$. Fix $V \in \mathcal{U}(G)$ and find $W \leqslant_{o} g^{-1} V g$ as given by the definition of $C \in \mathcal{H}$. So there is $\left(W_{i}\right)_{i \in \omega}$ a normal basis at 1 for $W$ and $N_{W} \unlhd W$ with $N_{W} \sim_{c} A$ such that $C \leqslant \bigcap_{i \in \omega} N_{G}\left(N_{W} \cap W_{i}\right)$. 
We claim $g W g^{-1} \leqslant_{o} V$ satisfies the conditions for $g C g^{-1} \in \mathcal{H}$. Certainly, $g N_{W} g^{-1} \unlhd$ $g W g^{-1},\left(g W_{i} g^{-1}\right)_{i \in \omega}$ is a normal basis at 1 for $g W g^{-1}$, and

$$
g C g^{-1} \leqslant \bigcap_{i \in \omega} N_{G}\left(g N_{W} g^{-1} \cap g W_{i} g^{-1}\right) .
$$

Since $g N_{W} g^{-1} \sim_{c} g A g^{-1}$ and $g A g^{-1} \sim_{c} A$, it is additionally the case that $g N_{W} g^{-1} \sim_{c} A$. Therefore, $\mathcal{H}$ is conjugation invariant.

We may now form $N_{\mathcal{H}}$, the $\mathcal{H}$-core.

Claim. $Z(A) \leqslant N_{\mathcal{H}}$.

Proof of Claim. Take $g \in Z(A)$ and $C \in \mathcal{H}$. Fix $V \in \mathcal{U}(G)$ and find $W \leqslant_{o} V$ as given by the definition of $C \in \mathcal{H}$. So there is $\left(W_{i}\right)_{i \in \omega}$ a normal basis at 1 for $W$ and $N_{W} \unlhd W$ with $N_{W} \sim_{c} A$ and $C \leqslant \bigcap_{i \in \omega} N_{G}\left(N_{W} \cap W_{i}\right)$. Since $N_{W} \sim_{c} A$, there is $i \in \omega$ such that $N_{W} \cap W_{i} \leqslant A \cap N_{W}$. Fix such an $i$ and put $N_{W_{i}}:=N_{W} \cap W_{i}$.

We now have that $N_{W_{i}} \unlhd W_{i}, N_{W_{i}} \sim_{c} A$, and $\left(W_{j}\right)_{j \geqslant i}$ is a normal basis at 1 for $W_{i}$. Since $N_{W_{i}} \leqslant A$, it is further the case that

$$
\overline{\langle C, g\rangle} \leqslant \bigcap_{j \geqslant i} N_{G}\left(N_{W_{i}} \cap W_{j}\right) .
$$

We conclude that $\overline{\langle C, g\rangle} \in \mathcal{H}$ and therefore, that $g \in N_{\mathcal{H}}$.

Put $N:=\overline{N_{\mathcal{H}}}$ and let $\pi: G \rightarrow G / N$ be the usual projection. Since $\operatorname{Comm}_{G / N}(\pi(B))=G / N$, the triple $(G / N, \pi(U), \pi(B))$ satisfies the hypotheses of the lemma. Moreover, $\pi(A) \leqslant_{o} \pi(B)$, $\pi(A) \unlhd \pi(U)$, and $n(\pi(A)) \leqslant k$. The induction hypothesis thus implies $G / N$ is elementary.

On the other hand, let $\left(n_{i}\right)_{i \in \omega}$ list a countable dense set of $N_{\mathcal{H}}$ and define

$$
P_{i}:=\left\langle U, n_{0}, \ldots, n_{i}\right\rangle .
$$

Since $U \in \mathcal{H}$ and $n_{0}, \ldots, n_{i} \in N_{\mathcal{H}}$, we have that $P_{i} \in \mathcal{H}$ for each $i$. Following from the definition of $\mathcal{H}$, we may find $W \leqslant_{o} U$ and $L \unlhd W$ such that $L \leqslant_{o} A$ and $P_{i} \leqslant N_{G}(L)$.

Lemma 9.6 implies $U / A$ has a dense quasi-centre, and since $W /(A \cap W) \leqslant_{o} U / A$, the group $W / A \cap W$ has a dense quasi-centre. Applying Lemma 9.6 to $W$ gives that $W / L$ also has a dense quasi-centre because $L \leqslant_{o} A \cap W$ and $L \unlhd W$. Letting $\pi: P_{i} \rightarrow P_{i} / L$ be the usual projection, we infer that

$$
\pi(W) \leqslant \overline{Q Z\left(P_{i} / L\right)} \leqslant \operatorname{SIN}\left(P_{i} / L\right),
$$

and therefore, $\operatorname{SIN}\left(P_{i} / L\right) \unlhd_{o} P_{i} / L$. It now follows that $P_{i}$ is elementary, and since $N \leqslant \bigcup_{i \in \omega} P_{i}$ is closed, Theorem 3.18 implies $N$ is also elementary.

The class $\mathscr{E}$ is closed under group extension, so we conclude that $G \in \mathscr{E}$ completing the induction.

Lemma 9.8. Suppose $G$ is a t.d.l.c.s.c. group and $U \in \mathcal{U}(G)$ contains a non-trivial virtually nilpotent $B \unlhd U$ such that $\operatorname{Comm}_{G}(B)=G$. Then $\overline{Q C}_{G}(U / B)$ is an elementary normal subgroup of $G$ containing $B$.

Proof. Put

$$
N:=\overline{Q C}_{G}(U / B):=\overline{\{g \in G \mid \exists W \in \mathcal{U}(G) \text { such that }[g, W \cap U] \subseteq B\}} .
$$


Proposition 2.8 implies $N$ is a normal subgroup containing $B$ since $B$ and $U$ are commensurated and $B \unlhd U$. Furthermore, $B \unlhd V:=U \cap N \in \mathcal{U}(N), Q Z(V / B)$ is dense in $V / B$, and $\operatorname{Comm}_{N}(B)=\bar{N}$. Lemma 9.7 therefore implies $N$ is also elementary.

We now present a slight adaptation of a lemma due to Caprace, Reid, and Willis from their work $[10$.

Let $G$ be a t.d.l.c. group, $U \in \mathcal{U}(G)$, and $K \unlhd U$ be infinite. Take $g \in G$ and consider $K^{g}:=$ $g K g^{-1}$. Certainly, $K^{g} \unlhd U^{g}$, and since $U$ is compact and open, there are $u_{1}, \ldots, u_{n}$ in $U$ such that $U \subseteq \bigcup_{i=1}^{n} u_{i} U^{g}$. For all $u \in U$, there is then some $1 \leqslant i \leqslant n$ such that $u K^{g} u^{-1}=u_{i} K^{g} u_{i}^{-1}$. Putting

$$
\left(K^{g}\right)^{U}:=\left\{u_{1} K^{g} u_{1}^{-1}, \ldots, u_{n} K^{g} u_{n}^{-1}\right\},
$$

the set of subgroups $\left(K^{g}\right)^{U}$ is permuted by $U$ under the action by conjugation.

Take $g_{1}, \ldots, g_{n} \in G$ and let $K^{h_{1}}, \ldots, K^{h_{m}}$ list $\{K\} \cup \bigcup_{i=1}^{n}\left(K^{g_{i}}\right)^{U}$. We now consider the group

$$
H:=\overline{\left\langle K^{h_{1}}, \ldots, K^{h_{m}}\right\rangle} .
$$

Since $U$ permutes the generating set, we immediately see that $U \leqslant N_{G}(H)$. Let us make a few further observations: Since $N_{G}\left(K^{h_{j}}\right)$ is open in $G$ for each $1 \leqslant j \leqslant m$, the subgroup $V:=$ $\bigcap_{j=1}^{m} N_{H \cap U}\left(K^{h_{j}}\right)$ is a compact open subgroup of $H$. We additionally have that $V \cap K^{h_{j}} \unlhd V$ for each $1 \leqslant j \leqslant m$, hence

$$
L:=\left(V \cap K^{h_{1}}\right) \ldots\left(V \cap K^{h_{m}}\right)
$$

is an infinite normal subgroup of $V$.

We claim that $L$ is also commensurated in $H$. Since $L \leqslant N_{H}\left(K^{h_{j}}\right)$, we have that $L K^{h_{j}}$ is a compact subgroup, and furthermore, $L K^{h_{j}} / L$ is finite as $\left(K^{h_{j}} \cap V\right) \leqslant L$ and $L K^{h_{j}} / L \leftrightarrow$ $K^{h_{j}} / L \cap K^{h_{j}}$. The group $L$ is a thus a finite index subgroup of $L K^{h_{j}}$, and it follows that $K^{h_{j}} \leqslant$ $\operatorname{Comm}_{H}(L)$. As $V \leqslant \operatorname{Comm}_{H}(L)$, we conclude that $\operatorname{Comm}_{H}(L)$ is a dense open subgroup of $H$ and therefore equals $H$. That is to say, $L$ is commensurated in $H$.

We have now demonstrated the following lemma:

Lemma 9.9 (Caprace, Reid, Willis [10]). Suppose $G$ is a t.d.l.c. group, $U \in \mathcal{U}(G), K \unlhd U$ is infinite, and $g_{1}, \ldots, g_{n} \in G$. Let $K^{h_{1}}, \ldots, K^{h_{m}}$ list $\{K\} \cup \bigcup_{i=1}^{n}\left(K^{g_{i}}\right)^{U}$ and put

$$
H:=\overline{\left\langle K^{h_{1}}, \ldots, K^{h_{m}}\right\rangle} \text {. }
$$

Then,

(1) $U \leqslant N_{G}(H)$, and

(2) for $V:=\bigcap_{j=1}^{m} N_{H \cap U}\left(K^{h_{j}}\right)$ and $L:=\left(V \cap K^{h_{1}}\right) \ldots\left(V \cap K^{h_{m}}\right)$, it is the case that $L \unlhd V \in$ $\mathcal{U}(H), L$ is infinite, and $\operatorname{Comm}_{H}(L)=H$.

\subsection{Structure theorems}

Lemma 9.10. Suppose $G$ is a t.d.l.c.s.c. group with trivial quasi-centre. If $G$ has a nontrivial locally normal $[A]$-subgroup and $U \in \mathcal{U}(G)$, then $\overline{Q C}_{G}(U /[A](U))$ is a non-trivial elementary normal subgroup.

Proof. Since $Q Z(G)$ is trivial, the non-trivial locally normal $[A]$-subgroup that $G$ is hypothesized to have must be infinite. It follows that $U$ has an infinite locally normal $[A]$ subgroup, and from Lemma 9.3 , we infer that $U$ has an infinite normal $[A]$-subgroup. Thus, 
$[A](U)$ is non-trivial. We now form

$$
N:=\overline{Q C}_{G}(U /[A](U)):=\overline{\{g \in G \mid \exists W \in \mathcal{U}(G) \text { such that }[g, W \cap U] \subseteq[A](U)\}} .
$$

By Proposition 9.4 $[A](U)$ is commensurated, hence Proposition 2.8 implies $N$ is a normal subgroup that contains $[A](U)$. It remains to show $N$ is elementary.

Let $\left\{A_{j}\right\}_{j \in J}$ list all normal $[A]$-subgroups of $U$ and let $\left(W_{i}\right)_{i \in \omega}$ be a normal basis at 1 for $[A](U)$. For each $i$, we may find $\Omega_{i}:=\left\{A_{j_{1}}, \ldots, A_{j_{n}}\right\} \subseteq\left\{A_{j}\right\}_{j \in J}$ so that $\bigcup \Omega_{i}$ contains coset representatives for $[A](U) / W_{i}$. Put $\Omega:=\bigcup_{i \in \omega} \Omega_{i}$, list $\Omega$ as $\left(B_{i}\right)_{i \in \omega}$, and for each $i \in \omega$, define $C_{i}:=B_{0} B_{1} \ldots B_{i}$. The sequence $\left(C_{i}\right)_{i \in \omega}$ is thus an $\subseteq$-increasing sequence of infinite closed normal virtually nilpotent subgroups of $U$ with $\bigcup_{i \in \omega} C_{i}$ dense in $[A](U)$.

Fix $\Sigma=\left\{g_{i} \mid i \in \omega\right\}$ a countable dense subgroup of $G$. For each $i \in \omega$, let $C_{i}^{h_{1}}, \ldots, C_{i}^{h_{m(i)}}$ list $C_{i},\left(C_{i}^{g_{0}}\right)^{U}, \ldots,\left(C_{i}^{g_{i}}\right)^{U}$ and define

$$
P_{i}:=\overline{\left\langle C_{i}^{h_{1}}, \ldots, C_{i}^{h_{m(i)}}\right\rangle}
$$

The construction of $P_{i}$ allows us to apply Lemma 9.9 giving $V \in \mathcal{U}\left(P_{i}\right)$ and

$$
\left(V \cap C_{i}^{h_{1}}\right) \ldots\left(V \cap C_{i}^{h_{m(i)}}\right)=L \unlhd V
$$

with $\operatorname{Comm}_{P_{i}}(L)=P_{i}$. Each $C_{i}$ is virtually nilpotent, so $L$ is also virtually nilpotent. Setting $N_{i}:=\overline{Q C}_{P_{i}}(V / L)$, Lemma 9.8 implies $N_{i}$ is an elementary normal subgroup of $P_{i}$.

On the other hand, let $\pi: P_{i} \rightarrow P_{i} / N_{i}=: \tilde{P}_{i}$ be the usual projection. We have that $N_{P_{i}}\left(C_{i}^{h_{j}}\right)$ is open in $P_{i}$ and thus, that $N_{\tilde{P}_{i}}\left(\pi\left(C_{i}^{h_{j}}\right)\right)$ is open in $\tilde{P}_{i}$ for each $1 \leqslant j \leqslant m(i)$. However, $\pi\left(C_{i}^{h_{j}}\right)$ is finite as $L \cap C_{i}^{h_{j}}$ is finite index in $C_{i}^{h_{j}}$, and this implies

$$
\pi\left(C_{i}^{h_{j}}\right) \leqslant Q Z\left(\tilde{P}_{i}\right) \leqslant \operatorname{SIN}\left(\tilde{P}_{i}\right) .
$$

for each $j$. It follows that $\operatorname{SIN}\left(\tilde{P}_{i}\right)=\tilde{P}_{i}$, so $\tilde{P}_{i}$ is elementary. Since $\mathscr{E}$ is closed under group extension, we conclude that $P_{i}$ is elementary.

The $P_{i}$ form an $\subseteq$-increasing sequence of elementary groups with $U \leqslant N_{G}\left(P_{i}\right)$ for each $i$. Lemma 3.7 thus implies $P:=\bar{\bigcup}_{i \in \omega} P_{i}$ is also elementary.

Claim. $P \unlhd G$.

Proof of Claim. It suffices to show the countable dense subgroup $\Sigma$ of $G$ used in the definition of $P$ normalizes $P$. Fixing $P_{j}$ from the construction of $P$ and $g \in \Sigma$, it is indeed enough to show $g P_{j} g^{-1} \subseteq P$.

Take $C_{j}^{u g_{k}}$ one of the generating subgroups from the construction of $P_{j}$. We may assume $u \in \Sigma$ since the normalizer of $C_{j}^{g_{k}}$ is open and $\Sigma$ is dense. So $g u g_{k} \in \Sigma$, and there is some sufficiently large $l$ such that $C_{j} \leqslant C_{l}$ and $g u g_{k}$ is in the first $l$ elements of the enumeration of $\Sigma$. Thus, $C_{l}^{g u g_{k}} \leqslant P_{l}$. Since $P_{j}$ is topologically generated by finitely many groups of the form $C_{j}^{u g_{k}}$, it follows that $g P_{j} g^{-1} \leqslant P_{l^{\prime}} \leqslant P$ for some large $l^{\prime}$ proving the claim.

By the claim and the construction of $P$, we now have that $[A](U) \leqslant P \unlhd N$. Let $\pi: N \rightarrow$ $N / P$ be the usual projection and put $W:=N \cap U$. The group $\pi(W)$ is a quotient of $W /[A](U)$ and, therefore, has a dense quasi-centre. It follows that $\operatorname{SIN}(N / P)$ is open in $N / P$, so $N / P \in \mathscr{E}$. Since $\mathscr{E}$ is closed under group extension, we conclude that $N=\overline{Q C}_{G}(U /[A](U))$ is elementary.

We now prove the main theorem of this section.

TheOREM 9.11. If $G$ is an $[A]$-regular t.d.l.c.s.c. group, then $G$ is elementary. 
Proof. Suppose $G$ is an $[A]$-regular group, so $G / \operatorname{Rad}_{\mathscr{E}}(G)$ is also $[A]$-regular. Suppose for contradiction $G / \operatorname{Rad}_{\mathscr{E}}(G)$ is non-trivial. We thus have that $G / \operatorname{Rad}_{\mathscr{E}}(G)$ is a non-discrete t.d.l.c.s.c. group with a trivial quasi-centre and a non-trivial locally normal $[A]$-subgroup. Fixing $U \in \mathcal{U}\left(G / \operatorname{Rad}_{\mathscr{E}}(G)\right)$, we apply Lemma 9.10 to conclude that $\overline{Q C}_{G / \operatorname{Rad}_{\mathscr{E}}(G)}(U /[A](U))$ is a non-trivial elementary normal subgroup of $G / \operatorname{Rad}_{\mathscr{E}}(G)$. This is absurd as $G / \operatorname{Rad}_{\mathscr{E}}(G)$ has a trivial elementary radical.

Corollary 9.12. If $G$ is a t.d.l.c.s.c. group, then $G / \operatorname{Rad}_{\mathscr{E}}(G)$ is $[A]$-semisimple. In particular, if $G$ is an elementary-free t.d.l.c.s.c. group, then $G$ is $[A]$-semisimple.

Proof. Certainly, $G / \operatorname{Rad}_{\mathscr{E}}(G)$ must have a trivial quasi-centre. Since $G / \operatorname{Rad}_{\mathscr{E}}(G)$ has no non-trivial elementary normal subgroups, Lemma 9.10 implies $G / \operatorname{Rad}_{\mathscr{E}}(G)$ has no non-trivial abelian locally normal subgroups. Therefore, $G / \operatorname{Rad}_{\mathscr{E}}(G)$ is $[A]$-semisimple.

Corollary 9.13. If $G$ is a t.d.l.c.s.c. group, then $R_{[A]}(G)$ is elementary.

Proof. By Corollary 9.12, $G / \operatorname{Rad}_{\mathscr{E}}(G)$ is $[A]$-semisimple, and Theorem 9.5 therefore implies $R_{[A]}(G) \leqslant \operatorname{Rad}_{\mathscr{E}}(G)$. Applying Theorem 3.18 , we conclude that $R_{[A]}(G)$ is elementary.

We lastly recover a theorem from the literature.

Corollary 9.14 (Caprace, Reid, Willis [10]). A non-discrete compactly generated topologically simple t.d.l.c. group is $[A]$-semisimple.

Proof. Via [16. (8.7)], such a group $G$ is second countable, so Proposition 6.3 implies $G$ is non-elementary. We conclude that $G$ is elementary-free and via Corollary 9.12 , is $[A]$ semisimple.

REMARK 9.15. In general, $R_{[A]}(G) \lesseqgtr \operatorname{Rad}_{\mathscr{E}}(G)$.

\section{References}

1. H. ABELS, 'Specker-Kompaktifizierungen von lokal kompakten topologischen Gruppen', Math. Z., 135 $(1973 / 74): 325-361$.

2. Y. Barnea, M. Ershov, and T. Weigel, 'Abstract commensurators of profinite groups', Trans. Amer. Math. Soc., 363 (2011)(10):5381-5417.

3. B. BeKka, P. De la Harpe, and A. Valette, Kazhdan's property (T), volume 11 of New Mathematical Monographs, (Cambridge University Press, Cambridge2008).

4. G. M. Bergman and H. W. Lenstra, Jr., 'Subgroups close to normal subgroups', J. Algebra, 127 $(1989)(1): 80-97$.

5. M. G. BRIN, 'Elementary amenable subgroups of R. Thompson's group F', Internat. J. Algebra Comput., $15(2005)(4): 619-642$.

6. M. Burger and S. Mozes, 'Groups acting on trees: from local to global structure', Inst. Hautes Études Sci. Publ. Math., (2000)(92):113-150 (2001).

7. P.-E. CAPrace and N. Monod, 'Relative amenability', ArXiv:1309.2890 [math.GR], http://arxiv.org/abs/1309.2890.

8. P.-E. Caprace and N. Monod, 'Decomposing locally compact groups into simple pieces', Math. Proc. Cambridge Philos. Soc., 150 (2011)(1):97-128.

9. P.-E. Caprace, C. Reid, and G. Willis, 'Locally normal subgroups of totally disconnected groups. Part I: General theory', ArXiv:1304.5144 [math.GR], http://arxiv.org/abs/1304.5144. 
10. P.-E. Caprace, C. Reid, and G. Willis, 'Locally normal subgroups of totally disconnected groups. Part II: Compactly generated simple groups', ArXiv:1401.3142 [math.GR], http://arxiv.org/abs/1401.3142.

11. C. Chou, 'Elementary amenable groups', Illinois J. Math., 24 (1980)(3):396-407.

12. J. Dieudonné, La géométrie des groupes classiques, (Springer-Verlag, Berlin1971), troisième édition, Ergebnisse der Mathematik und ihrer Grenzgebiete, Band 5.

13. S. GaO and M. Xuan, 'On non-Archimedean Polish groups with two-sided invariant metrics', Topology Appl., 161 (2014):343-353.

14. H. GLÖCKNER, 'Elementary p-adic Lie groups have finite constructible rank', ArXiv:1402.4919 [math.GR] http://arxiv.org/abs/1402.4919.

15. H. GlÖCKNer and G. Willis, 'Uniscalar p-adic Lie groups', Forum Math., 13 (2001)(3):413-421.

16. E. HewitT and K. Ross, Abstract harmonic analysis. Vol. I, volume 115 of Grundlehren der Mathematischen Wissenschaften [Fundamental Principles of Mathematical Sciences], (Springer-Verlag, Berlin1979), second edition.

17. G. Higman, 'A finitely generated infinite simple group', J. London Math. Soc., 26 (1951):61-64.

18. A. Kechris, Classical descriptive set theory, volume 156 of Graduate Texts in Mathematics, (SpringerVerlag, New York1995).

19. B. KröN and R. MÖLler, 'Analogues of Cayley graphs for topological groups', Math. Z., 258 (2008)(3):637675.

20. K. Kunen, Set theory, volume 102 of Studies in Logic and the Foundations of Mathematics, (North-Holland Publishing Co., Amsterdam1980), an introduction to independence proofs.

21. A. M. Macbeath and S. Świerczkowski, 'On the set of generators of a subgroup', Nederl. Akad. Wetensch. Proc. Ser. A 62 = Indag. Math., 21 (1959):280-281.

22. A. Y. Olshanskil and D. Osin, 'A quasi-isometric embedding theorem for groups', ArXiv:1202.6437 [math.GR] http://arxiv.org/abs/1202.6437.

23. D. V. Osin, 'Elementary classes of groups', Mat. Zametki, 72 (2002)(1):84-93.

24. V. P. Platonov, 'Locally projectively nilpotent subgroups and nilelements in topological groups', Izv. Akad. Nauk SSSR Ser. Mat., 30 (1966):1257-1274.

25. İ. V. Protasov and V. S. ČArīn, 'Projections of topological groups', Mat. Zametki, 24 (1978)(3):383-389, 447.

26. J. Tits, 'Sur le groupe des automorphismes d'un arbre', 'Essays on topology and related topics (Mémoires dédiés à Georges de Rham)', pages 188-211, (Springer, New York1970).

27. V. I. Trofimov, 'Graphs with polynomial growth', Mat. Sb. (N.S.), 123(165) (1984)(3):407-421.

28. P. WesOleK, 'A note on relative amenability', ArXiv:1406.2974 [math.GR], http://arxiv.org/abs/1406.2974.

29. P. WesoleK, 'Totally disconnected locally compact groups locally of finite rank', ArXiv:1406.1526 [math.GR], http://arxiv.org/abs/1406.1526.

30. G. WiLlis, 'Compact open subgroups in simple totally disconnected groups', J. Algebra, 312 (2007)(1):405417 .

\section{Phillip Wesolek}

MSCS University of Illinois at Chicago

322 Science and Engineering Offices MC249

851 S. Morgan St. Chicago, Il 60607-7045

USA

Current address:

Université catholique de Louvain IRMP

Chemin du Cyclotron 2, box L7.01.02

1348 Louvain-la-Neuve

Belgique

phillip.wesolek@uclouvain.be 